\title{
ANALYTICAL AND NUMERICAL OPTIMIZATION OF AN IMPLANTABLE VOLUME CONDUCTION ANTENNA
}

\author{
by \\ Brian Langan Wessel \\ BS, University of Pittsbugh, 2002
}

\author{
Submitted to the Graduate Faculty of \\ the School of Engineering in partial fulfillment \\ of the requirements for the degree of \\ Master of Sciences
}

University of Pittsburgh

2004 


\section{UNIVERSITY OF PITTSBURGH SCHOOL OF ENGINEERING}

This thesis was presented

by

Brian Langan Wessel

It was defended on

April 5, 2004

and approved by

Robert Sclabassi, Professor, Department of Neurological Surgery

Mingui Sun, Associate Professor, Department of Neurological Surgery

J. Robert Boston, Associate Chairman, Department of Electrical Engineering

Ching-Chung Li, Professor, Department of Electrical Engineering

Thesis Advisors: Robert Sclabassi, Professor, Department of Neurological Surgery,

Mingui Sun, Associate Professor, Department of Neurological Surgery 


\title{
ANALYTICAL AND NUMERICAL OPTIMIZATION OF AN IMPLANTABLE VOLUME CONDUCTION ANTENNA
}

\author{
Brian Langan Wessel, M.S. \\ University of Pittsburgh, 2004
}

As implantable devices become increasingly sophisticated, a means of communication is required to transmit data to and from the device. A volume conduction antenna model has been developed that meets the size and power constraints of an in vivo environment. This thesis aims to optimize the shape, curvature, and orientation of these antennas. Analytical and numerical analysis shows that the performance is independent of the conic section used to simulate an antenna. Both analyses were also in agreement that highest curvatures achieve maximum surface potentials, and that the angle is dependent on the distance of the antenna from the surface of the head. Analytical analysis suggests that pointing the antenna elements directly at the surface may not be the optimum angle, but rather at a smaller angle. Too few data points were taken to make the same determination from the numerical case but the optimum angle does deviate from the hypothesized angle in the same way, suggesting a similar result. The numerical analysis was important as it facilitated the simulation of the epoxy between the antenna elements. Incorporating epoxy into the simulation showed $30-35 \%$ increases in surface potential. A reflective sheet was then added showing further increases in surface potential. 


\section{PREFACE}

I would like to extend my sincere thanks to Dr. Sclabassi and Dr. Sun for their guidance, positive support, and continual attention to my growth and development as a student and researcher. They were always available to talk and spend as much time as I needed to make things clear.

I also thank the students in the Laboratory for Computational Neuroscience for listening to my many and diverse questions and willingness to lend-a-hand.

Special thanks to my family: Mom, Dad, Frannie, Heather, Brenda and John, Heidi and Tommy, Grandma and Grandpa, and everyone else for believing in me and always having words of support.

Last, but not least, I would like to thank my thesis committee for spending their time to read and listen to my thesis defense and offer constructive criticism to help stengthen the research. 


\section{TABLE OF CONTENTS}

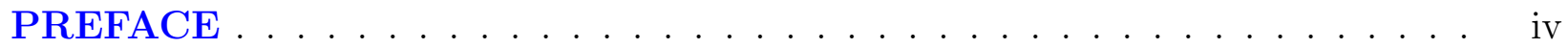

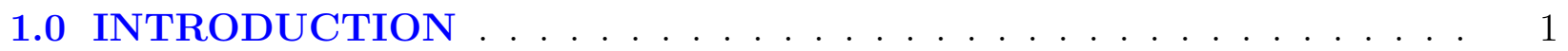

1.1 MOTIVATION ........................ 1

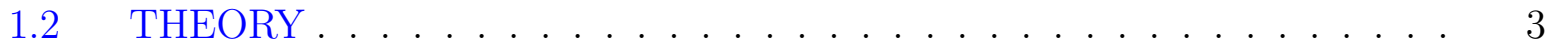

1.2 .1 Volume Conductor . . . . . . . . . . . . . . . . . . 3

1.2.1.1 Quasi-static Assumption . . . . . . . . . . . . . . 4

1.2.1.2 Attenuation . . . . . . . . . . . . . . 5

1.2.1.3 Half-cell Assumptions . . . . . . . . . . . . . . 5

1.2.2 The Finite Element Method $(\mathrm{FEM}) \ldots \ldots \ldots$

1.2.3 The Boundary Element Method (BEM) . . . . . . . . . . 8

1.2.4 Comparison of the FEM and BEM . . . . . . . . . . . 12

2.0 METHODS : ANALYTICAL AND NUMERICAL . . . . . . . . . . . 15

2.1 CONSTRUCTION OF THE ANTENNA ELEMENTS . . . . . . . . . . . 15

2.2 ANALYTICAL METHODS . . . . . . . . . . . . . . . . . 18

2.2.1 Ideal Current Dipoles in an Infinite Homogeneous Medium . . . . . 18

2.2.2 Nonideal Current Dipoles in an Infinite Homogeneous Medium . . . 21

2.2.3 Ideal Current Dipoles in a Spherical Homogeneous Medium . . . . . 22

2.2.4 Nonideal Current Dipoles in a Spherical Homogeneous Medium . . 22

2.2.5 Ideal Current Dipoles in a Four Shell Spherical Head Geometry . . 24

2.2 .6 Graphical User Interface . . . . . . . . . . . . . . . . . . 25

2.3 NUMERICAL METHODS . . . . . . . . . . . . . . . . . . . 27

2.3 .1 FEMLab . . . . . . . . . . . . . . . . . . . . . . . 27 
3.0 RESULTS

3.1 NONIDEAL DIPOLES IN A SPHERICAL MEDIUM: ANALYTICAL INVESTIGATION . . . . . . . . . . . . . . . . . . . . . . . 29

3.1.1 Investigation of Optimum Angle . . . . . . . . . . . . . . . 29

3.2 FINITE ELEMENT RESULTS: NUMERICAL INVESTIGATION . . . . 31

3.2 .1 Proof of Concept in 2D . . . . . . . . . . . . . . . . 31

3.2 .2 Proof of Concept in 3D . . . . . . . . . . . . . . . 35

3.2 .3 Finite Element Analysis in 3D . . . . . . . . . . . . . . 35

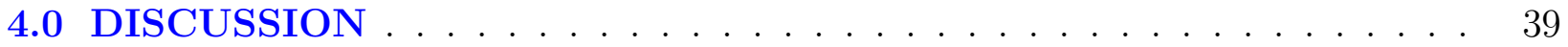

4.1 VERIFYING ANALYTICAL AND NUMERICAL RESULTS FOR THE 1-SHELL MODEL . . . . . . . . . . . . . . . . . . . . . . . . . 39

4.1.1 Comparing FE with Epoxy to Analytical Solutions . . . . . . . . 42

4.1 .2 Basis Functions . . . . . . . . . . . . . . . . . . 44

4.2 SAMPLING THE SURFACE OF THE SHAPES . . . . . . . . . . . . 48

4.3 CONVERGENCE OF THE SOLUTION VS. CONDUCTIVITY DIFFER-

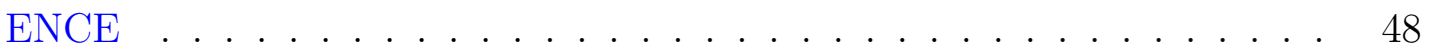

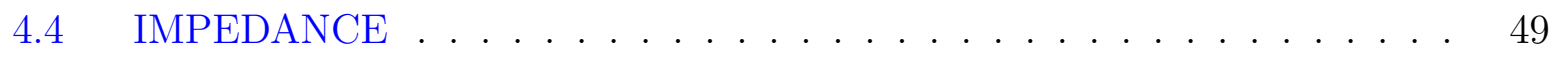

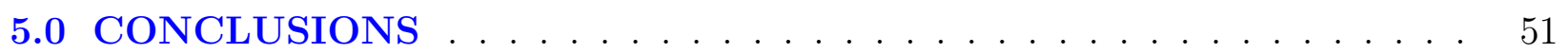

APPENDIX. MATLAB AND MEX CODE . . . . . . . . . . . . 52

$.1 \quad$ Graphical User Interface $\ldots \ldots \ldots$

.2 Shape Optimization . . . . . . . . . . . . . . . . . . . . . . 71

$.3 \quad$ FE shapes creation $\ldots \ldots \ldots \ldots \ldots \ldots$

.4 Four Shell Files . . . . . . . . . . . . . . . . . . . . . . . . . . . . 89

$.5 \quad$ FE vs Frank Equation . . . . . . . . . . . . . . . . . . . . . . . . 93

$.6 \quad$ Extracting Points FEMLab output $($ Ctrl-F) $\ldots \ldots \ldots$

$.7 \quad$ Ideal vs Non-Ideal Dipoles $(2 \mathrm{D} \infty$ medium $) \ldots \ldots \ldots$

.8 Ideal vs Non-Ideal Dipoles (3D spherical medium) . . . . . . . . . . . 101

$.9 \quad$ Hypothetical Angle . . . . . . . . . . . . . . . . . . . . . . . . . . . . . . 104

10 Extracting Points from Slices in 3D . . . . . . . . . . . . . . 109

.11 PDE Tool Code - Epoxy _ . . . . . . . . . . . . . . . . . . . 111 
.12 PDE Tool Code - No Epoxy _. . . . . . . . . . . . . . . . . . . . 119

.13 PDE Tool Code - Reflector . . . . . . . . . . . . . . . . . . . 125

.14 PDE Multishell (with epoxy) . . . . . . . . . . . . . . . . . 131

.15 Plotting - Epoxy with \% increase from analytical . . . . . . . . . . . 139

.16 Plotting - No epoxy (FE) with $\%$ increase from analytical . . . . . . . 143

.17 Conductivity Convergence . . . . . . . . . . . . . . . . . 147

BIBLIOGRAPHY . . . . . . . . . . . . . . . . . . . . . . . . . 149 


\section{LIST OF TABLES}

1 Resistivities of relevant material properties ( [7] compiled biological material) 3

2 Relevant Assumptions for Human Tissue $[10] \ldots \ldots$. . . . . . . . . . . 4

3 Results for Proofs of Concept . . . . . . . . . . . . . . . . . . . . 35 


\section{LIST OF FIGURES}

1 Antenna elements shown on the sides of the implantable device. . . . . . . . 2

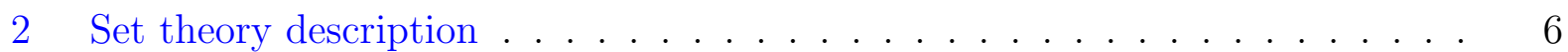

3 Top left: Illustrates the use of control points around a bounding box $(2 * x m a x$ wide and ymax high) to determine parameters of the specified shape. Top right: Shows a specific example of Top left using 5 control points. Bottom: Shows how the antenna elements can be rotated by an angle $\Psi$. . . . . . . . 16

4 Vector field of ideal dipoles evenly spaced on hyperbolically shaped antenna elements. The dipoles were placed in an orientation perpendicular to the antenna elements and evenly spaced with-respect-to the arclength. . . . . . . . 17

5 Dipole consisting of a $\operatorname{sink}-I_{o}$ at the origin and a source $I_{o}$ at $d \hat{a}_{d}$, where $\mathrm{d} \rightarrow 0$. Also illustrated is a field point at vector $r \hat{a}_{r}$ and polar angle $\theta[7] . .$.

6 Two dipoles shown in a 3D coordinate system. The primed variables are the locations of the dipoles and the unprimed variable is the location of interest. .

7 Top left: The field created by an ideal dipole. Top Right: Superposition of two ideal dipoles. Bottom Left: Two monopoles (source and sink). Bottom Right: Error between 2 dipoles and 2 monopoles (Appendix .7). . . . . . . . . . 23

8 Coordinate system illustrating the variables from Equation (2.28) . . . . . .

9 Shaded plot showing the percent error for two sets of dipoles as compared to one nonideal dipole. The error is concentrated in the region between the antenna elements as the approximation breaks down in the near-field (Appendix .8). . 
10 Graphical User Interface allowing user to specify antenna element shape, size, position, and orientation. It is also capable of simulating one or two dipoles alone by user positioning in polar coordinates (Appendix .1). . . . . . . . . 26

11 Various conic sections. Top left: Semicircular; Top right: Parabolic; Bottom left: Elliptical; Bottom right: Hyperbolic. . . . . . . . . . . . . . 26

12 An example of a finite element mesh . . . . . . . . . . . . . . . . . 28

13 Top left: Semicircular; Top right: Parabolical; Bottom left: Elliptical; Bottom right: Hyperbolic (Appendix .2) . . . . . . . . . . . . . .

14 As the antenna moves towards the surface, the optimum angle increases and is concave down. This graph is based on (3.1) and code is found in Appendix .9. 32

15 Shows that as the antenna is moved toward the surface, the optimum angle rotates so that the antenna elements will point towards the surface (Appendix $9) \ldots \ldots \ldots \ldots \ldots \ldots \ldots$

16 The antenna elements are simulated without an insulator between the elements. The polar plot displays the surface voltage. The right plot shows equipotential lines surrounding the antenna elements. The polar plot has units of Volts

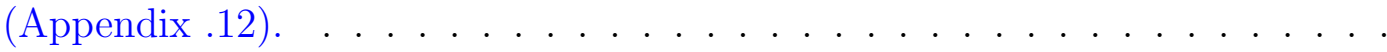

17 The antenna elements are simulated only with an insulator between the elements. The polar plot displays the surface voltage. The right plot shows equipotential lines surrounding the antenna elements. The polar plot has units of Volts (Appendix .11) . . . . . . . . . . . . . . .

18 An insulating material is placed below the antenna elements to act as a current reflector. The polar plot displays the surface voltage. The right plot shows equipotential lines surrounding the antenna elements. The polar plot has units of Volts (Appendix .13). . . . . . . . . . . . . . . . .

19 Depicts the 2 planes where the slices were taken. . . . . . . . . . . . . 36

20 The antenna elements are simulated without an insulator between the elements. The polar plot displays the surface voltage (Code to extract slices from FEMLab in Appendix .10). . . . . . . . . . . . . . . . . . . . 
21 The antenna elements are simulated without an insulator between the elements. The polar plot displays the surface voltage. . . . . . . . . . . . . . . 37

22 The antenna elements are simulated with an insulator between the elements. The polar plot displays the surface voltage. . . . . . . . . . . . . .

23 The antenna elements are simulated with an insulator between the elements. The polar plot displays the surface voltage. . . . . . . . . . . . . . . . 38

24 Results for the FEA with epoxy between the antenna elements. Top left: Semicircular Top right: Parabolical Bottom left: Elliptical Bottom right: Hyperbolic 38

25 Comparison of Finite Element and Frank equation solution for (nonideal dipoles) current sources around the center. The top plots show the potential plotted on the surface of a spherical model of the head. The bottom plots show the same voltage distribution plotted on a grid (Appendix .5) . . . . . . . .

26 Comparison of FEM and Frank equation solution for current sources near the surface. The top plots show the potential plotted on the surface of a spherical model of the head. The bottom plots show the same voltage distribution plotted on a grid. . . . . . . . . . . . . . . . .

27 Comparison of FEM and Frank equation solution for current sheets near the surface. . . . . . . . . . . . . . . . . .

28 Finite element solution for all shapes, angles and curvatures where there is no epoxy between the elements. These results should compare well with Figure 13. The error is shown in Figure 29 (Code found in Appendix .16). . . . . . .

29 Comparison of FEM and Frank equation solution for all shapes, angles and curvatures. The percent increase represents how different the finite element solution is compared to the analytical. . . . . . . . . . . . . . .

30 Comparison of FEM and Frank equation solution for all shapes, angles and curvatures with epoxy. The percent increase represents how the epoxy does not allow current to short between the antenna elements and thus forces it to go around the antenna and give better surface voltages (Appendix .15). . . .

31 The black line shows the maximum value for each column which is confined to only one row or in other words, one value of angle $\phi$ (Appendix .5) . . . . . 
32 Top Plot: Error. Bottom Plot: Error with a sine curve overlaid (Appendix .5). 45

33 Different angles of the voltages plotted on a rectangular grid (Appendix .5). . 46

34 Graph shows that for an increasing conductivity difference, the solution does not change (Appendix .17) . . . . . . . . . . . . . . . . . . 49

35 Maximum element voltage plotted versus angle and curvature with no epoxy between the antenna. . . . . . . . . . . . . . . . . 50

36 Maximum element voltage plotted versus angle and curvature with epoxy between the antenna. . . . . . . . . . . . . . . . . . . 50 


\subsection{INTRODUCTION}

\subsection{MOTIVATION}

In 1990 there were more than half a million people suffering epileptic seizures despite appropriate pharmacotherapy [2], and it has been estimated that 100,000 to 200,000 of these are potential surgical candidates [3]. For many of these people, their quality of life is severely diminished by the afflictions of their disease. Surgery has become an effective tool in ameliorating severe symptoms expressed by patients [4], however it can be difficult to ascertain the exact foci, or region of cells responsible for the epileptic expression. Operating procedures normally involve destruction of the suspect epileptic region. However, it would be beneficial to be able to control the epileptic region without destroying cells and/or monitor the region to better locate the foci. An implantable device could accomplish both of these tasks. Thus the device could be used in both long- and short-term settings.

Establishing volume conduction as a communication link would also benefit those with spinal cord injuries. This could address the problem of the means by which activity in the motor cortex would be coupled to muscle and how the sensory cortex could perceive the surroundings. In general, the device would be capable of stimulating any part of the brain. This feature could lead to next-generation devices capable of deep brain stimulation for patients with Parkinson's disease.

Thus, a need exists for bidirectional data communication between implantable and external devices, unconstrained by wires traversing the skin. Wires pose a problem as they can create possibilities of infection or they may break. Computational advantages arise as there is a nearly infinite amount of computing power outside of the body versus a limited amount

of such power inside due to the constraints of available space and energy. Peripheral comput- 


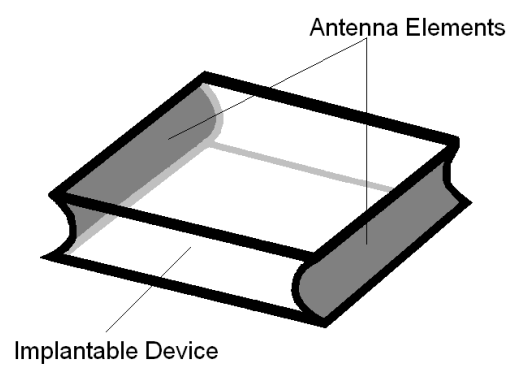

Figure 1: Antenna elements shown on the sides of the implantable device.

ing power can be concerned with problems of signal processing and other computationally intensive procedures, whereas an in vivo device(s) can perform very specialized tasks thus minimizing its size, weight, and energy consumption while greatly enhancing performance

Researchers have previously been successful in modeling these tissues as a volume conductor for the study of bioelectric events. We apply these models to design an efficient volume conduction antenna to both send and receive signals. Preliminary experimental results [5] have shown that two concave-shaped antenna elements located on the sides of an implantable device (Figure 1) is an efficient design. Further, it is hypothesized that the shape of the antenna elements will significantly affect how the current is distributed in the near- and far-field, as is the case in RF designs. However, volume conduction does differ from $\mathrm{RF}$ in the following ways [5]:

1. The strong shielding effect of ions in biological tissue is no longer a problem, instead, these ions are now employed as information carriers.

2. The electronic circuit associated with the communication system is simple and does not involve bulky components, allowing an aggressive reduction in size and weight.

3. The system does not require signal conversions to/from RF, increasing energy-efficiency.

Thus, volume conduction design differs from normal antenna design as it does not use RF energy but rather, volume currents. In RF design, the size of the antenna is dependent on the frequency of the signal. Further, to work in the frequency range having low attenuation, the frequency should be below $10 \mathrm{kHz}$ [23]. This results in an unreasonably large antenna size, on the order of $10^{4} \mathrm{~m}$. 


\subsection{THEORY}

\subsubsection{Volume Conductor}

Classical electrical engineering circuit design involves the analysis of networks of discrete components such as resistors, capacitors, inductors and sources. The investigation of these components led to constitutive relations thereby allowing the engineer to predict values in the circuit. However, the human body defies this discretization. The conducting medium can be thought of as a 3 -dimensional distributed circuit. This circuit contains no inductance but rather distributed resistance, capacitance, and sources [7].

Although the volume conductor is a distributed circuit, we must still ascertain and verify important material properties. First, the assumption of linearity is imposed. As a consequence, the rules of superposition and multiplication must hold. This is important so that the combined effects of current sources and sinks can be scaled and superposed. Although in reality, the properties of human tissue change depending on direction, these anisotropies are small for the tissue in the head, furthermore, in [7] only muscle tissue is cited as having significant anisotropies. Table 1 lists resistivity values for certain tissues.

Table 1: Resistivities of relevant material properties ( [7] compiled biological material)

\begin{tabular}{|c|c|c|}
\hline Material & Resistivity $\Omega \cdot m$ & References \\
\hline brain & 5.8 & average of [9] and [8] \\
\hline cerebral spinal fluid & 0.7 & {$[9]$} \\
\hline bone & 177 & {$[8]$} \\
\hline scalp & 2.22 & {$[1]$} \\
\hline epoxy (glass) & $1 \times 10^{12}$ & {$[1]$} \\
\hline metal (copper) & $1 \times 10^{-8}$ & {$[8$} \\
\hline
\end{tabular}

There are also ratios defined by [22] that specify the size of the brain, CSF, skull, and scalp with respect to the radius of the head. The brain surface is $88.24 \%$, CSF is $92.94 \%$, skull is $95.29 \%$ and scalp is $100 \%$ of the radius. Note that a radius of $7.5 \mathrm{~cm}$ was implemented. 
Table 2: Relevant Assumptions for Human Tissue [10]

\begin{tabular}{|c|c|}
\hline Condition & Criteria \\
\hline Neglect Propagation Effects & $k R_{\max } \ll 1$ \\
\hline Neglect Capacitive Effects & $\omega \epsilon / \sigma \ll 1$ \\
\hline Set $E_{1 n}=0$ & $\omega \epsilon_{0} / \sigma_{1}$ \\
\hline
\end{tabular}

1.2.1.1 Quasi-static Assumption Unlike in a discrete circuit, Kirchoff's laws cannot be applied here to solve a distributed circuit. Thus, one must solve partial differential equations for the resultant fields. Taking into account capacitance and propagation of the signal, one must solve the inhomogeneous Helmholtz equation to arrive at

$$
\Phi(\vec{r})=\frac{1}{4 \pi \sigma\left(1+\frac{j \omega \epsilon}{\sigma}\right)} \int_{V^{\prime}} \frac{\gamma^{\prime}\left(\vec{r}^{\prime}\right) e^{-j k R}}{R} d V^{\prime},
$$

where $R=|\vec{r}-\vec{r}|, \gamma^{\prime}$ is the volume current source density, $\sigma$ is the conductivity, and $k$ is a complex dielectric constant. To simplify this equation, a quasi-static assumption can be made which assumes that at each instant in time the potential field satisfies Poisson's equation, and that the boundary conditions are those which would exist if the source condition were stationary [10]. Plonsey showed that the assumptions in Table 2 are valid for frequencies below $1 \mathrm{kHz}$ for biological material. Using these conditions, taking the Laplacian of (1.1), and noting $\nabla^{2}(1 / R)=-4 \pi \delta\left(\vec{r}-\vec{r}^{\prime}\right)$, one arrives at Poisson's equation,

$$
\nabla^{2} \Phi(\vec{r})=-\frac{\gamma(\vec{r})}{\sigma}
$$

The boundary condition at the surface of the head must also be the same as that for the static case,

$$
\sigma_{1}\left(1+\frac{j \omega \epsilon_{1}}{\sigma_{1}}\right) E_{1 n}=\sigma_{2}\left(1+\frac{j \omega \epsilon_{2}}{\sigma_{2}}\right) E_{2 n}
$$

where $\sigma_{1}$ and $\sigma_{2}$ are the inner and outer conductivites, respectively, and $E_{1 n}$ and $E_{2 n}$ are the inner and outer electric fields, respectively. Thus, for $\sigma_{2}=0$ and $\epsilon_{2}=\epsilon_{0}$ the equation 
reduces to (1.4), where from the third entry in Table 2, one can arrive at the conclusion that $E_{1 n}=0$, which is exactly the same as the static case.

$$
\sigma_{1}\left(1+\frac{j \omega \epsilon_{1}}{\sigma_{1}}\right) E_{1 n}=j \omega \epsilon_{0} E_{2 n}
$$

1.2.1.2 Attenuation In order to maintain a suitable SNR, the frequency range should have low attenuation. Lindsey has shown that the frequency range below $10 \mathrm{kHz}$ has constant attenuation of about $54 \mathrm{~dB}$ for a current of $1 \mathrm{~mA}$. Frequencies above $10 \mathrm{kHz}$ exhibit an increase of $2 \mathrm{~dB} /$ decade to $100 \mathrm{kHz}$ [23]. These experiments were performed in the knee so more work must be done to characterize attenuation in the head.

1.2.1.3 Half-cell Assumptions Unlike a classical circuit, the conductance in a volume conductor arises due to ionic currents rather than electronic ones. Therefore, the antenna elements also play the role of transduction because the current in the antenna is carried by electrons whereas the current carried by the tissue is ions. In the case of the antenna, there will be currents flowing to and from the surface of the electrode. However, the half-cell potential, describing the transduction of electrons to ions, is calculated for a zero-current situation. Thus since the antenna violates the zero-current assumption, the half-cell potential must be modified by three terms to properly predict the behavior of the antenna element. These correction terms are called the ohmic, concentration, and activation overpotentials and describe the differences seen between the equilibrium zero-current and the observed half-cell

potential when the current is nonzero [11]. It will be assumed that these effects cancel as a negative effect on one electrode will be a positive effect on the other [6].

\subsubsection{The Finite Element Method (FEM)}

The FEM can be summarized in the following statement: Project the weak (variational) form of the differential equation onto a finite-dimensional function space [18]. The FEM is useful for solving partial differential equations (PDE). A PDE is an equation involving a function and its partial derivatives. 


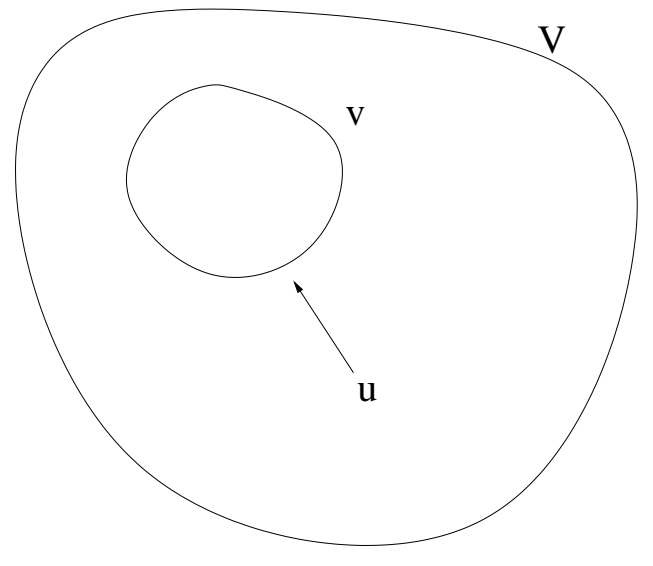

Figure 2: Set theory description

The derivation will be shown for three-dimensions but is appropriate for any number of dimensions. It is easiest to understand this statement by working through an example using the elliptic PDE, shown in the following equation:

$$
-\vec{\nabla} \cdot(\sigma \vec{\nabla} u)+a u=f \quad \text { in } \Omega \quad
$$

where $u$ is a scalar field, $a$ is a constant, $\sigma$ is the conductivity, which in general can be a matrix, and $f$ is a source term. Note that when $a$ is set equal to $0,(1.5)$ becomes the Poisson equation, (1.2). The first step is to project (1.5) onto a subspace of $V$, where it is contained. This subspace, of dimension $N$, also lies in $V$. This procedure is illustrated in Figure 2. Mathematically, this is shown in the following equation (please note that $d^{3} x$ is a volume integral, $d^{2} x$ is a surface integral, and $d x$ is a line integral):

$$
\int_{\Omega}\left(-\sigma \nabla^{2} \hat{u}\right) v+a \hat{u} v d^{3} x=\int_{\Omega} f v d^{3} x
$$

where $\hat{u}$ is an approximation to the solution, $u$. Green's first identity, shown in the following equation, is then applied:

$$
\int_{\Omega}\left(v \nabla^{2} \hat{u}+\vec{\nabla} v \vec{\nabla} \hat{u}\right) d^{3} x=\oint_{d \Omega} v \frac{\partial \hat{u}}{\partial n} d^{2} x
$$


Equation (1.6) becomes

$$
\int_{\Omega}(\sigma \vec{\nabla} \hat{u}) \cdot \vec{\nabla} v+a \hat{u} v d^{3} x-\oint_{d \Omega} \sigma \frac{\partial \hat{u}}{\partial n} v d^{2} x=\int_{\Omega} f v d^{3} x
$$

The following boundary condition is then applied:

$$
\sigma \frac{\partial \hat{u}}{\partial n}+q \hat{u}=g
$$

where when $\mathrm{q}$ and $\mathrm{g}$ are equal to zero is equivalent to writing:

$$
\frac{\partial u}{\partial r}=\left.0\right|_{r=R}
$$

In this case, Neumann boundary conditions were used on the outer boundary of the geometry. In general, Neumann conditions are always used on the inner surfaces. The result of substituting (1.9) into (1.8) is the following:

$$
\int_{\Omega}(\sigma \vec{\nabla} \hat{u}) \cdot \vec{\nabla} v+a \hat{u} v d^{3} x+\oint_{d \Omega}(q \hat{u}-g) v d^{2} x=\int_{\Omega} f v d^{3} x
$$

The original problem can thus be rephrased as the following: Find $u$ such that

$$
\left(\int_{\Omega}(c \vec{\nabla} \hat{u}) \cdot \vec{\nabla} v+a \hat{u} v-f v d x-\oint_{d \Omega}(-q \hat{u}+g) v d s\right)=0 \quad \forall v \quad
$$

Equation (1.12) is called the variational or weak form of the differential equation. As previously mentioned, $u$ and $v$ belong to some function space $V$. The next step is to determine a finite-dimensional subspace $v_{N} \subset V$. To project the weak form of the differential equation onto a finite-dimensional function space means requesting $u$ and $v$ to lie in $v_{N}$. Since (1.12) is true for all $v$, it is useful to define $N$ basis functions that $\operatorname{span} v$, thus $\phi_{i} \in v_{N}$. One can also expand $u$ in the same basis:

$$
\hat{u}(x)=\sum_{j=1}^{N} U_{j} \phi_{j}(x)
$$

This is known as Galerkin's method. Substituting for $\hat{u}$ and $v$ one gets 


$$
\sum_{j=1}^{N}\left(\int_{\Omega}\left(\sigma \vec{\nabla} \phi_{j}\right) \cdot \vec{\nabla} \phi_{i}+a \phi_{j} \phi_{i} d^{3} x+\oint_{d \Omega} q \phi_{j} \phi_{i} d^{2} x\right) U_{j}=\int_{\Omega} f \phi_{i} d^{3} x+\oint_{d \Omega} g \phi_{i} d^{2} x \quad i, \ldots, N
$$

Using the following notations:

$$
\begin{gathered}
K_{i, j}=\int_{\Omega}\left(\sigma \vec{\nabla} \phi_{j}\right) \cdot \vec{\nabla} \phi_{j} d^{3} x, \\
M_{i, j}=\int_{\Omega} a \phi_{j} \phi_{i} d^{3} x, \\
Q_{i, j}=\int_{d \Omega} q \phi_{j} \phi_{i} d^{2} x, \\
F_{i}=\int_{\Omega} f \phi_{i} d^{3} x \quad, \text { and } \\
G_{i}=\int_{d \Omega} g \phi_{i} d^{2} x,
\end{gathered}
$$

Equation (1.14) can be written in a compact matrix form:

$$
(\stackrel{\leftrightarrow}{K}+\overleftrightarrow{M}+\overleftrightarrow{Q}) \vec{U}=\vec{F}+\vec{G}
$$

Usually it is not necessary to distinguish between $\stackrel{\leftrightarrow}{K}, \overleftrightarrow{M}$, and $\overleftrightarrow{Q}$ or $\vec{F}$ and $\vec{G}$ so (1.20) can be written as:

$$
\stackrel{\leftrightarrow}{K} \vec{U}=\vec{F}
$$

\subsubsection{The Boundary Element Method (BEM)}

The boundary element method is similar to the FEM, however it seeks to find a relation

involving only the surface distributions of the unknown function $u, \frac{\partial u}{\partial n}$ and $u$ evaluated at a point $\mathrm{P}$ on the surface. Volume points can then be reconstructed if needed as a postprocessing step. Much of the material describing the BEM was adapted from [19]. 
Before one applies the BEM to a problem they must first find a fundamental solution, which becomes the weighting function, and plays a similar role as a particular solution in differential equations. It is also commonly called the freespace Green's function. This function can also be thought of as the response a system has to a $\delta$-function input.

Equation (1.2) will be solved with $\gamma$ set equal to zero i.e., a fundamental solution of Laplace's equation will be found. The solution will be valid in $\Re^{3}$. The fundamental solution of the Laplace equation is a solution of:

$$
\frac{\partial^{2} u}{\partial x^{2}}+\frac{\partial^{2} u}{\partial y^{2}}+\frac{\partial^{2} u}{\partial z^{2}}+\delta(\xi-x, \eta-y, \gamma-z)=0
$$

The method is to try and find a solution to $\nabla^{2} u=0$ in $\Re^{3}$ which contains a singularity at the point $(\xi, \eta, \gamma)$. It is expected that the solution is symmetric around the point $(\xi, \eta, \gamma)$ since $\delta(\xi-x, \eta-y, \gamma-z)$ is symmetric about this point. A local spherical coordinate system is adopted about the singular point $(\xi, \eta, \gamma)$. Letting $r=\sqrt{(\xi-x)^{2}+(\eta-y)^{2}+(\gamma-z)^{2}}$, we have

$$
\nabla^{2} u=\frac{1}{r^{2}} \frac{\partial}{\partial r}\left(r^{2} \frac{\partial u}{\partial r}\right)+\frac{1}{r^{2} \sin \theta} \frac{\partial}{\partial \theta}\left(\sin \theta \frac{\partial u}{\partial \theta}\right)+\frac{1}{r^{2} \sin \theta} \frac{\partial^{2} \psi}{\partial \phi^{2}}
$$

Note that the second and third terms are equal to zero due to symmetry, therefore $\frac{1}{r^{2}} \frac{\partial}{\partial r}\left(r^{2} \frac{\partial u}{\partial r}\right)=0$. This equation can be solved by simple integration:

$$
\int \frac{1}{r^{2}} \frac{\partial}{\partial r}\left(r^{2} \frac{\partial u}{\partial r}\right) r^{2} d r=0
$$

Canceling the $r^{2}$ variables, and applying the Fundamental Theorem of Calculus i.e., $\left(\int g^{\prime}(x) d x=g(x)+D\right)$ with an arbitrary constant $D$ :

$$
r^{2} \frac{\partial u}{\partial r}+D=0
$$

and redefining $-D$ as $A$ and integrating:

$$
\int \partial u=\int \frac{A}{r^{2}} \partial r
$$


where an arbitrary constant $B$ is used:

$$
u=\frac{A}{r}+B
$$

We must now find the constants $\mathrm{A}$ and $\mathrm{B}$. To do this we can make use of the integral property of the $\delta$-function. From (1.22) we must have

$$
\int_{\Omega} \nabla^{2} u=-\int_{\Omega} \delta d \Omega=-1 \text { where } \Omega \text { is any domain containing } r=0 \quad .
$$

It is wise to choose a simple domain to allow us to evaluate the integrals. If $\Omega$ is a small sphere of radius $\epsilon>0$ centered at $r=0$, then according to (1.7), with $\Phi=1$

$$
\int_{\Omega} \nabla^{2} u=\int_{\partial \Omega} \frac{\partial u}{\partial n} d^{2} x
$$

where $\partial \Omega$ is a surface integral. Furthermore, $n$ and $r$ are in the same direction, so

$$
\int_{\Omega} \nabla^{2} u=\int_{\partial \Omega} \frac{\partial u}{\partial r} d^{2} x
$$

Integrating around the perimeter, where $\frac{\partial u}{\partial r}=-\frac{A}{r^{2}}$, we get

$$
\int_{0}^{2 \pi} \int_{0}^{\pi}-\frac{A}{r^{2}}\left(r^{2} \sin \theta d \theta d \phi\right)=-4 \pi A
$$

Using (1.28), $A=\frac{1}{4 \pi}$. So we have

$$
u=\frac{1}{4 \pi r}+B
$$

where $B$ is arbitrary but usually set equal to zero. The foundation is now laid to develop the boundary element equation. The basic steps are quite similar to those used in the FEM. We begin with Green's second theorem:

$$
\int_{\Omega}\left(v \nabla^{2} u-u \nabla^{2} v\right) d^{3} x=\oint_{d \Omega}\left[v \frac{\partial u}{\partial n}-u \frac{\partial v}{\partial n}\right] d^{2} x \quad,
$$

where $\nabla^{2} u=0$. For the Galerkin FEM we chose $v$, the weighting function, to be $\phi_{j}$, one of the basis functions used to approximate $u$. For the BEM we choose $v$ to be the fundamental solution of Laplace's equation derived in (1.32) i.e., $v=\frac{1}{4 \pi r}$. 
Then using the property of the Dirac delta:

$$
\int_{\Omega}\left(u \nabla^{2} v\right) d^{3} x=-\int_{\Omega} u \delta(\xi-x, \eta-y, \gamma-z) d^{3} x=-u(\xi, \eta, \gamma) \quad(\xi, \eta, \gamma) \in \Omega
$$

i.e., the domain integral has been replaced by a point value. Thus (1.33) becomes

$$
u(\xi, \eta, \gamma)+\oint_{d \Omega} u \frac{\partial v}{\partial n} d^{2} x=\oint_{d \Omega} v \frac{\partial u}{\partial n} d^{2} x \quad(\xi, \eta, \gamma) \in \Omega
$$

This equation contains only boundary integrals (and no domain integrals as in the FEM). It relates the value of $u$ at some point inside the solution domain to integral expressions involving $u$ and $\frac{\partial u}{\partial n}$ over the boundary of the solution domain. It is usually more helpful to have an expression relating the value of $u$ at some point on the boundary to boundary integrals. For brevity, the result will not be derived but is as follows:

$$
c(P) u(P)+\oint_{d \Omega} u \frac{\partial v}{\partial n} d^{2} x=\oint_{d \Omega} v \frac{\partial u}{\partial n} d^{2} x \quad(\xi, \eta, \gamma) \in \Omega
$$

where:

$$
c(P)= \begin{cases}1 & \text { if } \mathrm{P} \in \Omega, \\ \frac{1}{2} & \text { if } \mathrm{P} \in d \Omega \text { and } d \Omega \text { smooth at } \mathrm{P}, \\ \frac{\text { inner solid angle }}{4 \pi} & \text { if } \mathrm{P} \in d \Omega \text { and } d \Omega \text { not smooth at } \mathrm{P} .\end{cases}
$$

Once the surface distributions of $u$ and $\frac{\partial u}{\partial n}$ are known, the value of $u$ at any point $\mathrm{P}$ inside $\Omega$ can be found since all surface integrals in (1.36) are known. Thus we solve for the boundary data first, and find the volume data as a separate step.

Since (1.36) contains only surface integrals, as opposed to volume integrals in a finite element formulation, the overall size of the problem has been reduced by one dimension. This can result in huge savings for a problem with a large volume to surface ratio. Also the effort required to produce a volume mesh of a complex three-dimensional object is far greater than that required to produce a mesh of the surface. Thus the BEM offers distinct advantages over the FEM in certain situations. There are also disadvantages, and both will be discussed in the next section. 


\subsubsection{Comparison of the FEM and BEM}

For complex geometries, the forward problem is typically solved by the BEM or FEM which solve for the potential on a 3D grid of data points. It is useful to compare the advantages and disadvantages of each method [16], [19].

\section{Meshing}

- FEM: An entire domain mesh is required.

- BEM: A mesh of the boundary only is required.

- Comment: The reduction in the size of the mesh implies that the problem complexity has been reduced by one dimension. This is advantageous as the creation of complex 3-dimensional meshes is time consuming. However, the FEM allows for the modeling of complex geometries.

\section{Solution Domain}

- FEM: The entire domain solution is calculated as part of the solution.

- BEM: The solution on the boundary is calculated first, and then the solution at domain points (if required) are found as a separate step.

- Comment: There are many problems where the details of interest occur on the boundary or are localized to a particular part of the domain, and hence an entire domain solution is not required. For problems involving infinite or semi-infinite domains, the BEM is favored as solving the whole domain is intractable.

\section{Approximations}

- FEM: The differential equation is being approximated.

- BEM: Boundary conditions are being approximated.

- Comment: The use of Green's second identity and a fundamential solution in the formulation means that the BEM involves no approximations of the differential equation in the domain; only in its approximations of the boundary conditions.

\section{Matrix Form}

- FEM: Sparse, banded, positive-definite, symmetric matrices are generated. 
- BEM: Fully populated nonsymmetric matrices generated.

- Comment: The two methods generally produce matrices of different sizes due to the differences in size of the domain mesh compared to the surface mesh. There are problems where either method can give rise to the smaller system and quickest solution, depending partly on the volume to surface ratio. For problems involving infinite or semi-infinite domains, the BEM is to be favored.

\section{Numerical Integration}

- FEM: Element integrals are easy to evaluate.

- BEM: Integrals are more difficult to evaluate, and some contain integrands that become singular.

- Comment: In general, BEM integrals are harder to evaluate. Also the integrals that are the most difficult (those containing singular integrands) have a significant effect on the accuracy of the solution, so these integrals need to be evaluated accurately.

\section{Applicability}

- FEM: Widely applicable. Handles nonlinear problems well.

- BEM: Cannot even handle all linear problems.

- Comment: A fundamental solution must be found (or at least an approximate one) before the BEM can be applied. There are many linear problems (e.g., virtually any nonhomogeneous equation) for which fundamental solutions are not known. There are certain areas in which the BEM is clearly superior, but it can be rather restrictive in its applicability.

\section{Implementation}

- FEM: Relatively easy to implement.

- BEM: Much more difficult to implement.

- Comment: The need to evaluate integrals involving singular integrands makes the BEM at least an order of magnitude more difficult to implement than a corresponding finite element procedure. 
In general, the FEM requires more computation. However, as computers increase in speed, this advantage diminishes and the FEM becomes more attractive for the advantages listed above. In addition, there are many more standard FEM programs to choose, therefore increasing user support. The BEM also works poorly in applications involving a large number of shells which makes it especially restrictive in this case where more realistic head geometries typically include 3 to 4 shells. For these reasons and the advantages above, the FEM was chosen as the numerical tool for these simulations. 


\subsection{METHODS : ANALYTICAL AND NUMERICAL}

\subsection{CONSTRUCTION OF THE ANTENNA ELEMENTS}

The construction of the antenna elements is illustrated in Figure 3. The same techniques were used to create both the analytical and numerical antenna shapes. The control points were found by adding $x_{\max }$ and $y_{\max }$ and dividing the sum by the number of control points $\left(P_{c}\right)$ plus one:

$$
d_{i n c}=\frac{x_{\max }+y_{\max }}{P_{c}+1} .
$$

This gives the incremental distance to travel along the perimeter of the bounding box as seen on the right side of Figure 3. The following give the equations for the sphere, parabola, ellipse, and hyperbola respectively.

$$
\begin{aligned}
& \frac{x^{2}}{1}+\frac{y^{2}}{1}=R^{2} \\
& a \frac{x^{2}}{1}-\frac{y}{1}=0 \\
& \frac{x^{2}}{a^{2}}+\frac{y^{2}}{b^{2}}=1 \quad, \text { and } \\
& \frac{y^{2}}{b^{2}}-\frac{x^{2}}{a^{2}}=1
\end{aligned}
$$

Thus we need to know $R$ for the sphere, $a$ for the parabola, and $a$ and $b$ for the ellipse and hyperbola. The following equations derive the formulas used to calculate the salient 


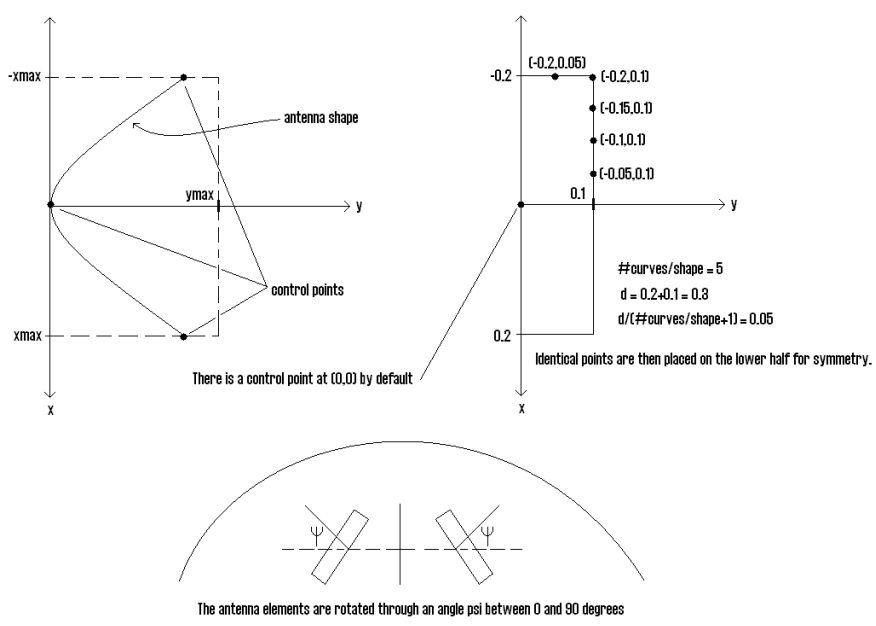

Figure 3: Top left: Illustrates the use of control points around a bounding box $\left(2^{*}\right.$ xmax wide and ymax high) to determine parameters of the specified shape. Top right: Shows a specific example of Top left using 5 control points. Bottom: Shows how the antenna elements can be rotated by an angle $\Psi$.

parameters of the conic section equations. For the sphere, $R$ can be calculated with the following equation, where the subscript, $c$, stands for "control points" as per Figure 3:

$$
R=\sqrt{x_{c}^{2}+y_{c}^{2}} \quad .
$$

For the parabola, $a$ can be calculated using

$$
a=\frac{y_{c}^{2}}{x_{c}} \quad .
$$

For the ellipse, it is assumed that the $x$ and $y$ control points are at the inflection points, thus

$$
\begin{gathered}
a=x_{c} \quad, \text { and } \\
b=y_{c} \quad .
\end{gathered}
$$




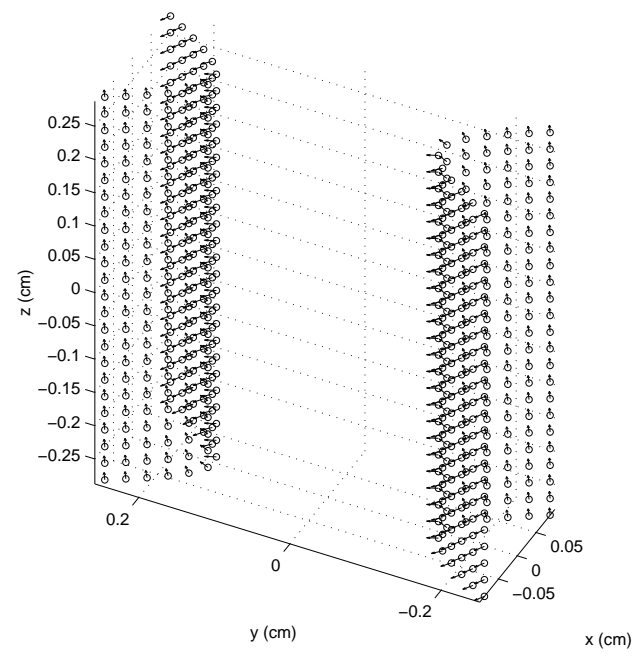

Figure 4: Vector field of ideal dipoles evenly spaced on hyperbolically shaped antenna elements. The dipoles were placed in an orientation perpendicular to the antenna elements and evenly spaced with-respect-to the arclength.

The hyperbola is a distance $\frac{d}{2}$ from the x-axis, where $d$ is specified by the user when designing the distance between the antenna. Thus,

$$
\frac{y^{2}}{\left(\frac{d}{2}\right)^{2}}-\frac{x^{2}}{a^{2}}=1
$$

where

$$
b=\frac{d}{2} \quad .
$$

Solving for $a^{2}$ we find

$$
a^{2}=\frac{y_{c}^{2} b^{2}}{x_{c}{ }^{2}+d x_{c}} .
$$

For the ideal dipole case, the dipoles have moments which are vectors, so a position and orientation must be specified. It was determined that putting the vectors at a perpendicular orientation to the surface would be consistent with the notion that the current leaves the surface of the antenna elements in a perpendicular direction. Determining this orientation 
is done by simply taking the gradient of the function set equal to zero:

$$
\begin{gathered}
y-f(x)=0 \\
\vec{m}_{d}=\vec{\nabla}(y-f(x))
\end{gathered}
$$

An example of the application of (2.13) and (2.14) can be seen in Figure 4. The sources and sinks were placed at evenly spaced intervals along the arc length of the shape. This same length was also used as the spacing along the long axis. Had the sources been placed at even intervals along the axis, the density of sources and sinks around the areas of high curvature would have been exaggerated. Thus, it was assumed that the current density leaving the antenna elements was uniform.

\subsection{ANALYTICAL METHODS}

Analytical methods are important because they offer fast, closed-form solutions. Methods were developed that allow the antennas to be simulated with both ideal and nonideal current dipoles. Nonideal current dipoles are characterized by having an arbitrary distance between source and sink monopoles.

\subsubsection{Ideal Current Dipoles in an Infinite Homogeneous Medium}

Ideal current monopoles can be characterized by defining the current magnitude and conductivity as seen in the following equation [7]:

$$
\Phi_{I_{o}}=\frac{I_{o}}{4 \pi \sigma r}
$$

The field produced by a current monopole and the electrostatic field from a point charge are identical, provided that $I_{o}$ is replaced by $Q_{o}, \sigma$ is replaced by $\epsilon$ (the permittivity), and 


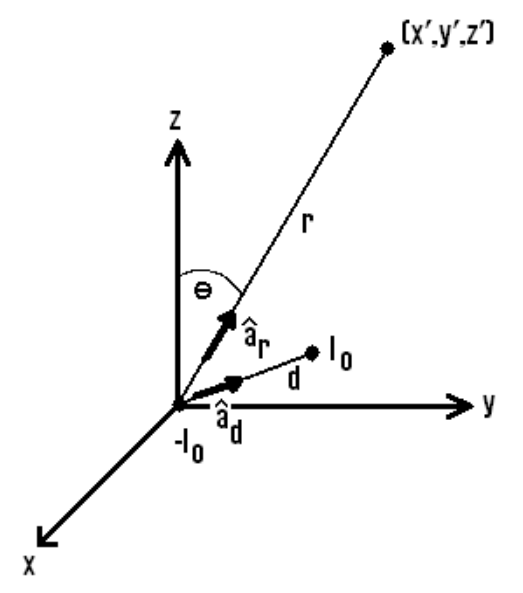

Figure 5: Dipole consisting of a sink $-I_{o}$ at the origin and a source $I_{o}$ at $d \hat{a}_{d}$, where $\mathrm{d} \rightarrow 0$. Also illustrated is a field point at vector $r \hat{a}_{r}$ and polar angle $\theta[7]$.

$\vec{J}$ (current density) replaced by $\vec{E}$. The following equation shows this similarity:

$$
\Phi_{Q_{o}}=\frac{Q_{o}}{4 \pi \epsilon_{o} r}
$$

This example of duality is convenient as there are many closed-form equations for electrostatic point charges that can be converted to electrostatic point current sources. Although, a point current source cannot exist, the potential can be calculated from the presence of a current sink and source together [7].

The ideal dipole equation for an infinite homogeneous medium will be derived here, and the results will be used to determine whether it is a good or bad assumption to place ideal dipoles on the surface of the antenna elements. This is of interest, as equations have previously been derived for sophisticated models of the geometry of the head i.e., ideal dipoles in four-shell models of the head.

A dipole of arbitrary orientation is illustrated in Figure 5, where the negative pole is placed at the origin of the coordinate system. If the positive pole were at the origin, the potential would be zero. Thus, the dipole potential arises due to the separation of the positive and negative poles. This potential can be found by examining the potential for the positive monopole and evaluating the change in potential brought about by moving the the 
monopole from the origin to its dipole position. This can be approximated from the first derivative of the potential. A derivative of $\Phi$ is taken with respect to the direction $\hat{a}_{d}$ (a directional derivative) and then multiplied by the magnitude, $d$. Thus, we have

$$
\Phi_{d}=\frac{\partial\left(\frac{I_{o}}{4 \pi \sigma r}\right)}{\partial a_{d}} d \quad .
$$

The directional derivative in (2.17) equals the gradient in the direction $\vec{a}_{d}$ so that

$$
\Phi_{d}=\nabla\left(\frac{I_{o}}{4 \pi \sigma r}\right) \cdot d \hat{a}_{d} \quad,
$$

and finally, since $I_{o} d=p$ :

$$
\Phi_{d}=\frac{p}{4 \pi \sigma} \nabla\left(\frac{1}{r}\right) \cdot \hat{a}_{d} \quad .
$$

Using the relation

$$
\nabla \frac{1}{r}=\frac{\hat{a}_{r}}{r^{2}} \quad,
$$

(2.19) becomes

$$
\Phi_{d}=\frac{p}{4 \pi \sigma r^{2}} \hat{a}_{r} \cdot \hat{a}_{d} \quad .
$$

Equation (2.21) can be simplified further given that the dipole is oriented along the $\mathrm{z}$-axis and located at the origin [7]:

$$
\Phi_{d}=\frac{p \cos \theta}{4 \pi \sigma r^{2}} \quad .
$$

The reason that an ideal dipole was investigated first, rather than two monopoles, is that neuronal activity in the brain is typically modeled by a configuration of ideal dipoles. Thus, it was a simple extension to set up a model which incorporated ideal dipoles. For instance, to implement these ideal current dipoles, a program was used, written by Sun for EEG analysis (Appendix .1).

Conceptually it is clear that the use of two sets of dipoles will not return the same solution as using two monopoles (source and sink). The following set of equations derives 


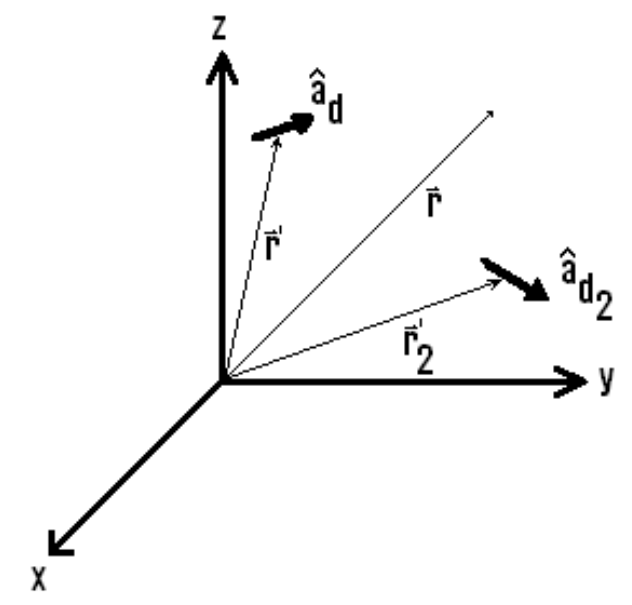

Figure 6: Two dipoles shown in a 3D coordinate system. The primed variables are the locations of the dipoles and the unprimed variable is the location of interest.

the field for two sets of dipoles in an infinite homogeneous medium. The coordinate system can be seen in Figure 6 .

Starting from (2.21) and using superposition, one can write the following equation for the second dipole, where here it is assumed that the moments $(p)$ are constant:

$$
\Phi_{2 d i p o l e s}=\frac{p}{4 \pi \sigma x^{2}} \hat{a}_{x} \cdot \hat{a}_{d}+\frac{p}{4 \pi \sigma x_{2}^{2}} \hat{a}_{x_{2}} \cdot \hat{a}_{d_{2}}
$$

where:

$$
x=\left|\vec{r}-\vec{r}^{\prime}\right| \quad,
$$

and

$$
x_{2}=\left|\vec{r}-{\overrightarrow{r_{2}}}^{\prime}\right| \quad,
$$

where the primed variables represent the location of the dipoles.

\subsubsection{Nonideal Current Dipoles in an Infinite Homogeneous Medium}

Nonideal current dipoles are classified as a set of opposing monopoles spaced at arbitrary distances. Each monopole obeys the laws of superposition and therefore the fields can simply 
be added together. Beginning with (2.15) and Figure 6, using superposition and rearranging, one can arrive at [7]:

$$
\Phi_{t o t}=\frac{I_{o}}{4 \pi \sigma}\left(\frac{1}{x_{2}^{\prime}}-\frac{1}{x^{\prime}}\right)
$$

where $x^{\prime}$ and $x_{2}^{\prime}$ are from (2.24) and (2.25), respectively.

Then comparing (2.23) and (2.26), one can find the error that arises from using two dipoles as compared to two monopoles for the antenna elements. This is shown in Figure 7. One can see that the error is quite large and so ideal dipoles are not an acceptable means of simulation in this case. Therefore, the previous literature for describing bioelectric sources as ideal dipoles in the head is not applicable for these simulations.

Although the equations for the infinite medium are insightful and provide a minimum baseline for error, they do not elucidate the characteristics of the dipoles inside of the head. To simulate the head, a spherical shape is assumed for the next section.

\subsubsection{Ideal Current Dipoles in a Spherical Homogeneous Medium}

There are many equations that have been developed to solve this problem. Probably the simplest equation was developed by Sidman [12]:

$$
\Phi=\frac{1}{4 \pi \sigma q} \sum_{i=1}^{3} m_{i}\left[\frac{2\left(e_{i}-d_{i}\right)}{q^{2}}+e_{i}+\frac{e_{i} s-d_{i}}{q+1-s}\right]
$$

where $d_{i}, m_{i}$, and $e_{i}, \mathrm{i}=1,2,3(1=\mathrm{x}, 2=\mathrm{y}, 3=\mathrm{z})$, are, respectively, the vector elements of $\vec{d}$, $\vec{m}$, and $\vec{e}$ which represent the dipole location, current moment, and scalp location; $\sigma$ and $\phi$ are the conductivity and potential at $\vec{e}$, respectively; and $\mathrm{q}$ and $\mathrm{s}$ are equal to $|\vec{e}-\vec{d}|$ and $\vec{e} \cdot \vec{d}$, respectively.

\subsubsection{Nonideal Current Dipoles in a Spherical Homogeneous Medium}

To increase simulation sophistication nonideal dipoles in a spherical medium were used. The derivation of this equation is quite complicated, using Legendre polynomials. It was 

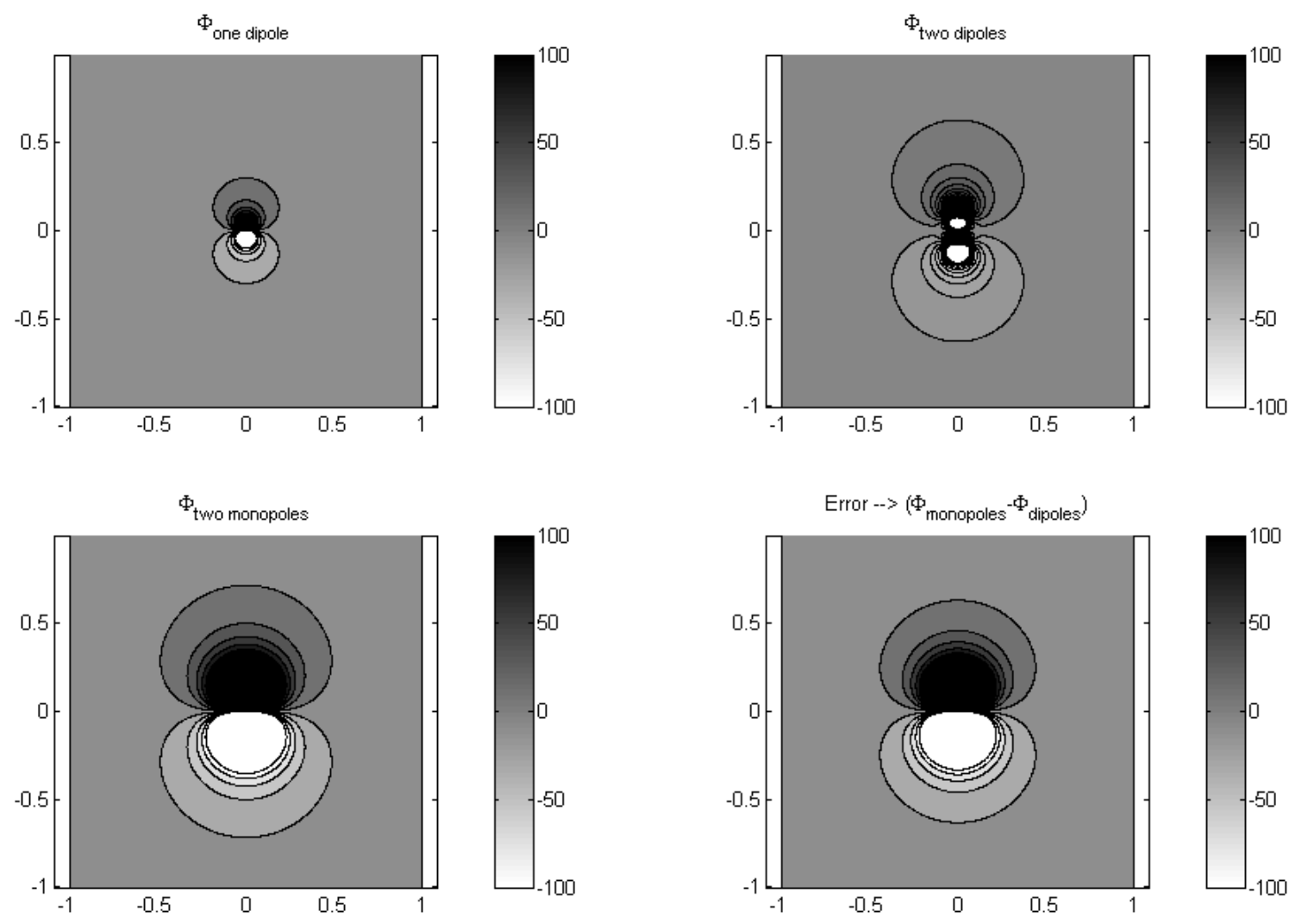

Figure 7: Top left: The field created by an ideal dipole. Top Right: Superposition of two ideal dipoles. Bottom Left: Two monopoles (source and sink). Bottom Right: Error between 2 dipoles and 2 monopoles (Appendix .7). 


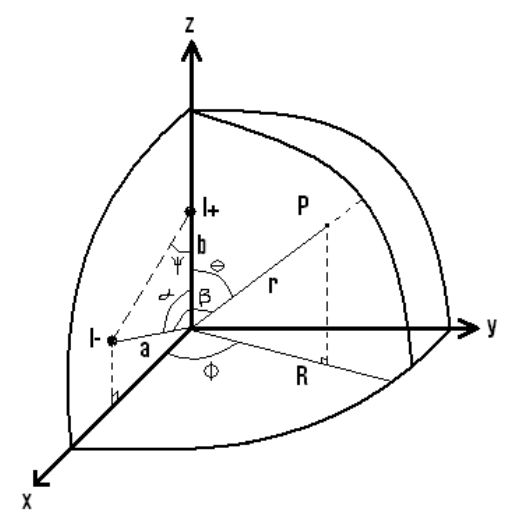

Figure 8: Coordinate system illustrating the variables from Equation (2.28)

developed by Ernest Frank [13] and is

$$
\phi(\theta, \beta)=\frac{I}{4 \pi \sigma}\left[\frac{2}{r_{b}}+\frac{2}{r_{a}}+\frac{1}{R} \ln \frac{r_{a}+R-a * \cos \beta}{r_{b}+R-b * \cos \theta}\right],
$$

where $r_{b}$ and $r_{a}$ extend from the positive monopole to the field point and from the negative monopole to the field point, respectively.

It is easiest to illustrate the variables before they are described as shown in Figure 8 From Figure 8 one can see that $d$ does not have to go to zero and thus this is a nonideal dipole arrangement inside of a spherical homogeneous medium.

With (2.27) and (2.28) one can find the error created by using dipoles. This error can be seen in Figure 9. Looking at this error, one can draw the conclusion that although (2.27) is quite useful for representing electrical activity due to neurons, it is not ideal for the closed-form simulation of the antenna.

\subsubsection{Ideal Current Dipoles in a Four Shell Spherical Head Geometry}

A four shell model of the head is important because it incorporates the different layers of tissue. The different tissues layers are brain, cerebral spinal fluid (CSF), bone, and scalp. Their conductivities are given in Table 1. The closed-form computation of the voltage in this case can be computationally intensive, but fortunately Sun found a compact mathematical solution which can be run efficiently in $\mathrm{C}$ [15]. Although the source model is an ideal dipole, 

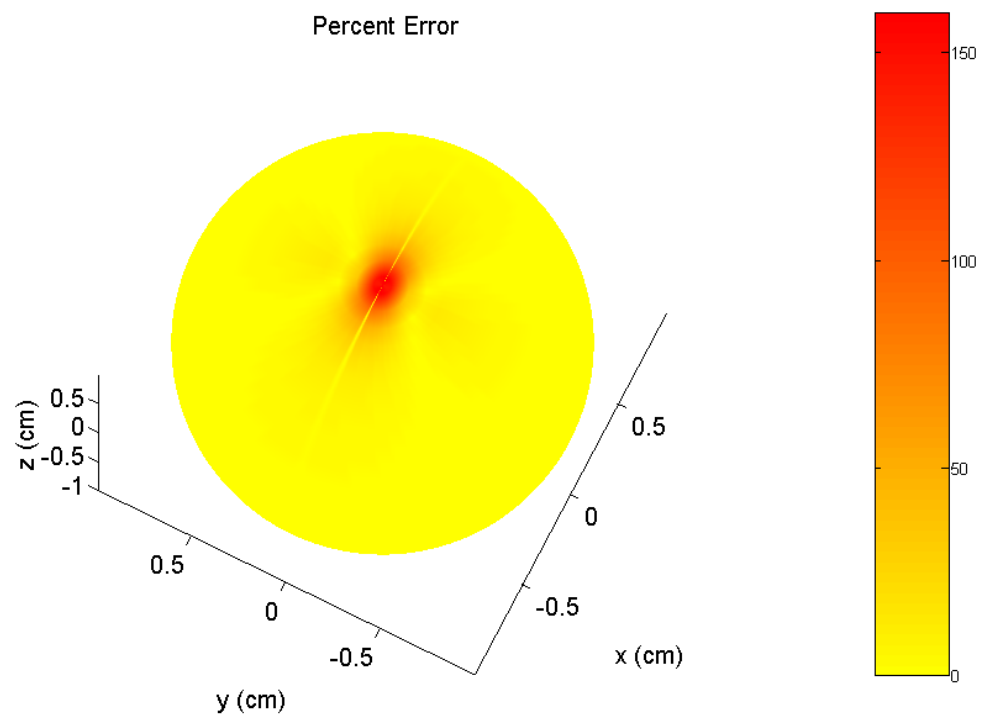

Figure 9: Shaded plot showing the percent error for two sets of dipoles as compared to one nonideal dipole. The error is concentrated in the region between the antenna elements as the approximation breaks down in the near-field (Appendix .8).

the program still holds value as it can be used to compare an ideal dipole arrangement of current sources and sinks on the antenna elements to FEM four shell results. A Mex function was written to interface this code with MatLab script (Appendix .4).

\subsubsection{Graphical User Interface}

Initially it was difficult to visualize how the antenna was to be placed inside the head and how the antenna elements would be simulated. It was decided that a graphical user interface (Figure 10) would aid in this process. The total current traversing from the source to sink was set to be $1 \mathrm{~mA}$. Because it was hypothesized that the shape of the antenna would affect the signal strength in both the near and far field, it was decided that conic sections would provide a good framework to test the shapes of the antennas. Figure 11 shows a semicircle, parabola, ellipse, and hyperbola.

Because the shapes have subtle differences, care was taken so that the dipoles were oriented perpendicularly to the surface of the antenna elements. Further, the dipoles were 

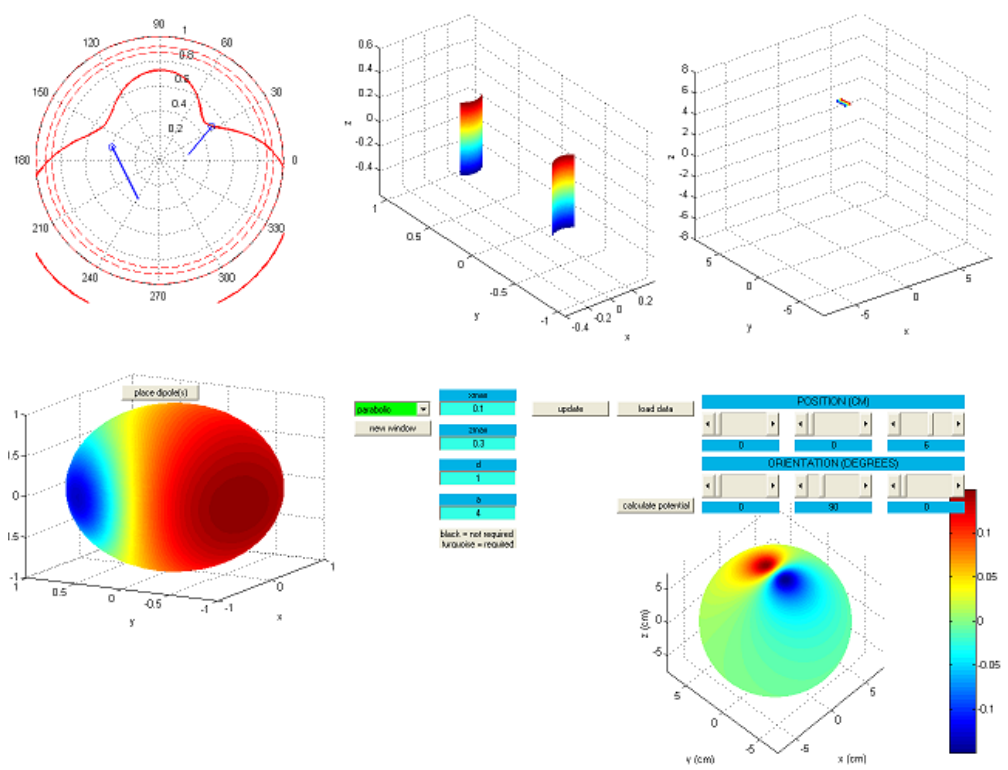

Figure 10: Graphical User Interface allowing user to specify antenna element shape, size, position, and orientation. It is also capable of simulating one or two dipoles alone by user positioning in polar coordinates (Appendix .1).
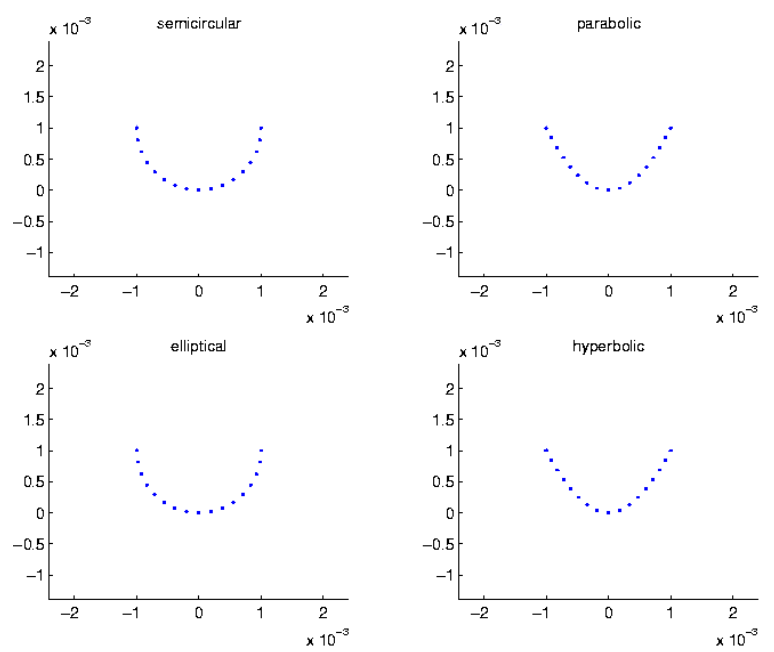

Figure 11: Various conic sections. Top left: Semicircular; Top right: Parabolic; Bottom left: Elliptical; Bottom right: Hyperbolic. 
placed so that the current density would be constant. Figure 4 shows how these dipoles were placed. For the semicircle the increment along the arc length could be calculated easily, but for the parabola, ellipse and hyperbola an iterative method was used to specify dipole placement. One interesting question is how many points is one required to seed on the shape so that the results will reflect the different shapes. This aspect of the model is a problem of sampling. The fewer the samples, the less computation needed to arrive at a solution. A suitable solution to this problem has not been worked out at this point. A frequency domain representation of the curves may lead to insights into the minium number of points required to characterize the shape. To circumvent this problem, the number of monopoles was simply made very dense so that this would not be a factor.

\section{$2.3 \quad$ NUMERICAL METHODS}

Numerical methods offer a way to simulate more complex geometries. Rarely can a closedform equation be derived to represent a real system. The numerical analysis was carried out in FEMLab (Finite Element Method Laboratory). This program runs together with MatLab and uses many similar features.

\subsubsection{FEMLab}

FEMLab has many modules for finite element solving (FEMLab standard package, Electromagnetics, Structural Mechanics, and Chemical Engineering). Initially it was thought that the Electromagnetics module would be required but it was found that the module did not have any increased functionality but only compiled commonly used finite element techniques from the FEMLab standard package. The FEMLab module contains an application mode called Conductive Media DC, which gives the user the ability to solve (1.2) for current sources. If $\sigma$ is a tensor, (1.2) is written as:

$$
\vec{\nabla} \cdot \overleftrightarrow{\sigma} \vec{\nabla} \Phi=-\gamma
$$

Equations (1.2) and (2.29) mean that the divergence of the electric field is nonzero where 


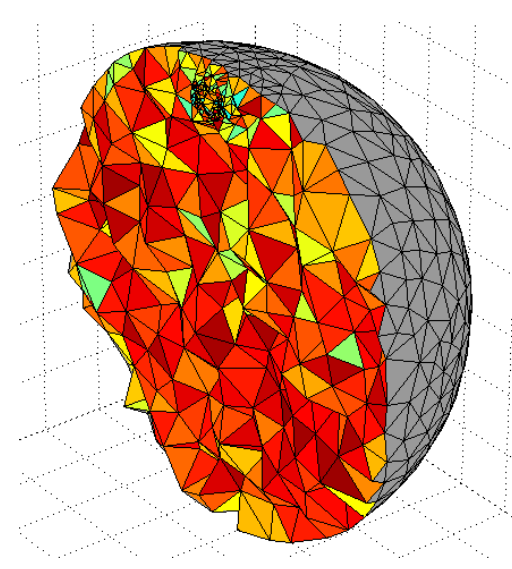

Figure 12: An example of a finite element mesh

there are current sources. After discretizing the Poisson equation over a tetrahedral mesh domain, the divergence of the electric field is approximated by (1.21) where $\overleftrightarrow{K}$ is typically called the stiffness matrix, $\vec{U}$ is a vector composed of voltages at the nodes, and $\vec{F}$ is a source vector indicating the flux through the nodes where it is only nonzero for nodes that are corners of elements containing a current source.

For the boundary conditions, equation (1.10) is utilized by FEMLab. The sphere and the geometry within the sphere is separated into subdomains. For each subdomain a conductivity and source current density can be specified. For the sphere and the epoxy the source current density is set equal to zero. For the antenna elements the source current density is set such that $1 \mathrm{~mA}$ will be sent and received. The values of the conductivities for the subdomains was set according to Table 1 . The geometry is meshed using a seven step process which is transparent to the user [14]. Figure 12 shows an example of the resulting mesh.

For this problem the Good Broyden iterative solver was used to solve (1.21). I will not go into the details of this, but normally these solvers are used to solve linear systems with a positive definite matrix $\stackrel{\leftrightarrow}{K}$, however for this condition to be true, the matrix must be preconditioned. Incomplete LU factorization was used in this case to give $\stackrel{\leftrightarrow}{K}$ the appropriate properties to be solved effectively by the Good Broyden method [14]. 


\subsection{RESULTS}

\subsection{NONIDEAL DIPOLES IN A SPHERICAL MEDIUM: ANALYTICAL INVESTIGATION}

The following results were obtained using Equations (2.6)-(2.12) to specify the antenna element points. These points were then plugged into (2.28). The z-axis shows the maximum potential found on the surface of the sphere, the y-axis the orientation of the antenna, and the $\mathrm{x}$-axis an index of the relative curvature of the shape. To describe how the curvature index is measured, Figure 3 shows five control points, therefore, in 1the curvature has an index from 1 to 5 . Notice that the circle only has 6 curvature indices, whereas the others have 10. This is because the curvature is uniform over the surface of the circle and is proportional

to $\frac{1}{R}$. Therefore, as the curvature is increased, the circle becomes smaller and smaller. For indices higher than 6, the shape defined was too small to cross through the control points so these points were not simulated. The more indices used to describe the curvature, the higher the resolution of the results, however since the results are not changing quickly, it was found that 10 control points was sufficient for the results of Figure 13.

\subsubsection{Investigation of Optimum Angle}

From the previous section one can see that the optimum angle seems to be around 45 degrees. Further, it makes sense that this angle would be a function of the distance that the antenna is from the surface. For instance, if the antenna was at the middle, it would make sense that the optimum angle is 0 degrees. Further, if the antenna was near the surface, the optimum angle should be steeper because the antenna elements would have to rotate to point at the 

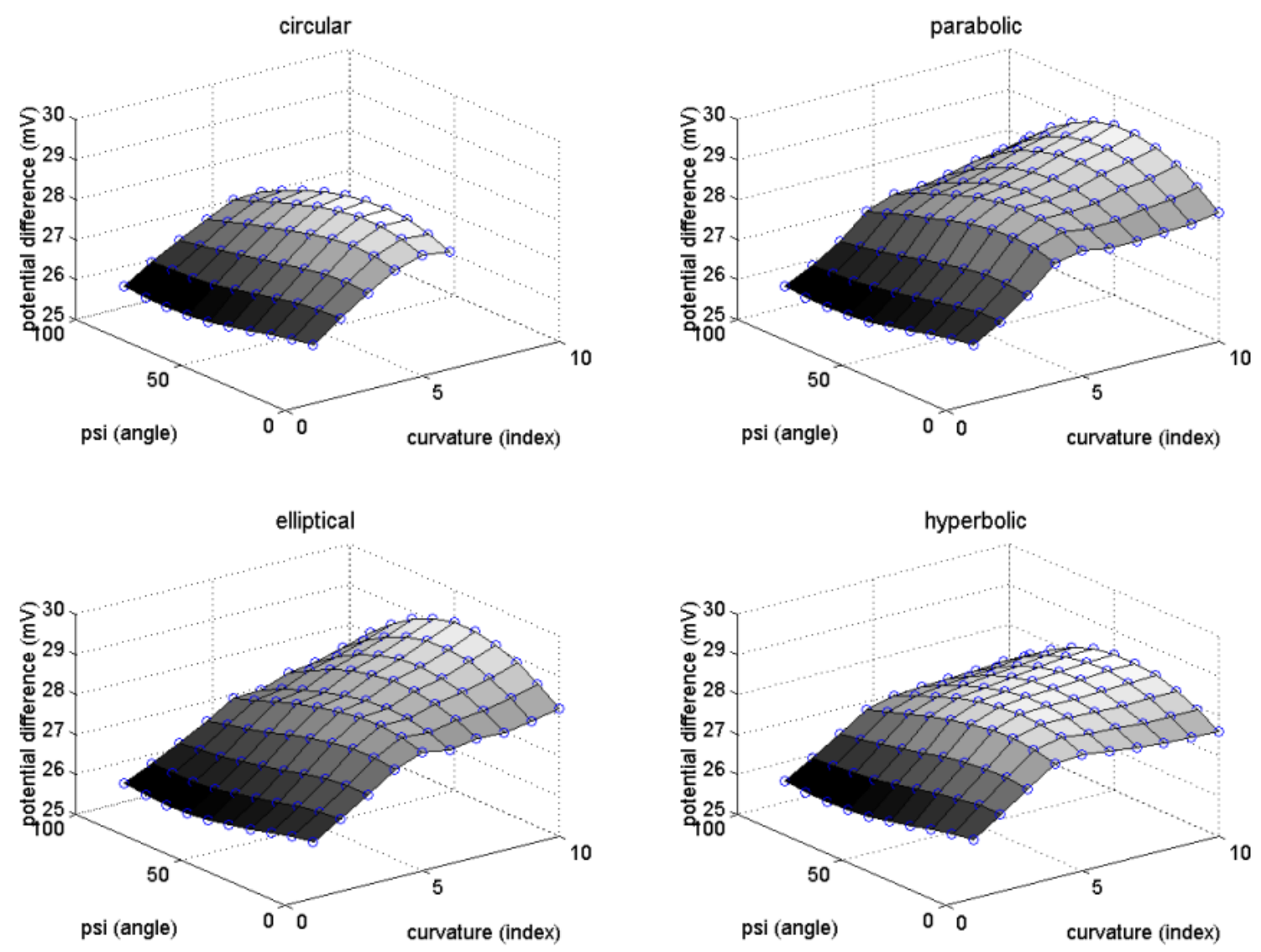

Figure 13: Top left: Semicircular; Top right: Parabolical; Bottom left: Elliptical; Bottom right: Hyperbolic (Appendix .2) 
surface. A hypothetical optimum angle was derived purely based on distance:

$$
\Psi_{\text {hypothetical }}=\arctan \left(\frac{y}{d / 2}\right)
$$

and a plot is shown in Figure 15. The results in Figure 15 agree with (3.1) in the sense that the angle does increase, however the actual curve is concave up. This indicates that the antenna elements destructively interfere with the generated surface voltages, requiring the antenna elements to be rotated away from each other.

\subsection{FINITE ELEMENT RESULTS: NUMERICAL INVESTIGATION}

\subsubsection{Proof of Concept in 2D}

To show proof of concept that the far field can be affected by near field changes, a 2D numerical analysis was done comparing:

1. Antenna elements without epoxy (Figure 16).

2. Antenna elements with epoxy (Figure 17).

3. Antenna elements with epoxy and a reflector below (Figure 18).

Equipotential lines are plotted for all three with polar plots adjacent showing the potential on the surface. Note that the absolute value of the voltage was taken so that it could be plotted in polar coordinates. Notice the voltage on the surface is lower for the case without epoxy between the elements. Therefore, an insulator between the elements is important for higher transmission efficiency. Notice also that without the reflector, the equipotential lines are nearly symmetric from the top to the bottom of the antenna, however, with the reflector, the equipotential lines are skewed towards the surface, thus improving directivity of the antenna, proving that the reflector can interact with the near field and thus alter the far field. This also translates into a higher surface potential. 


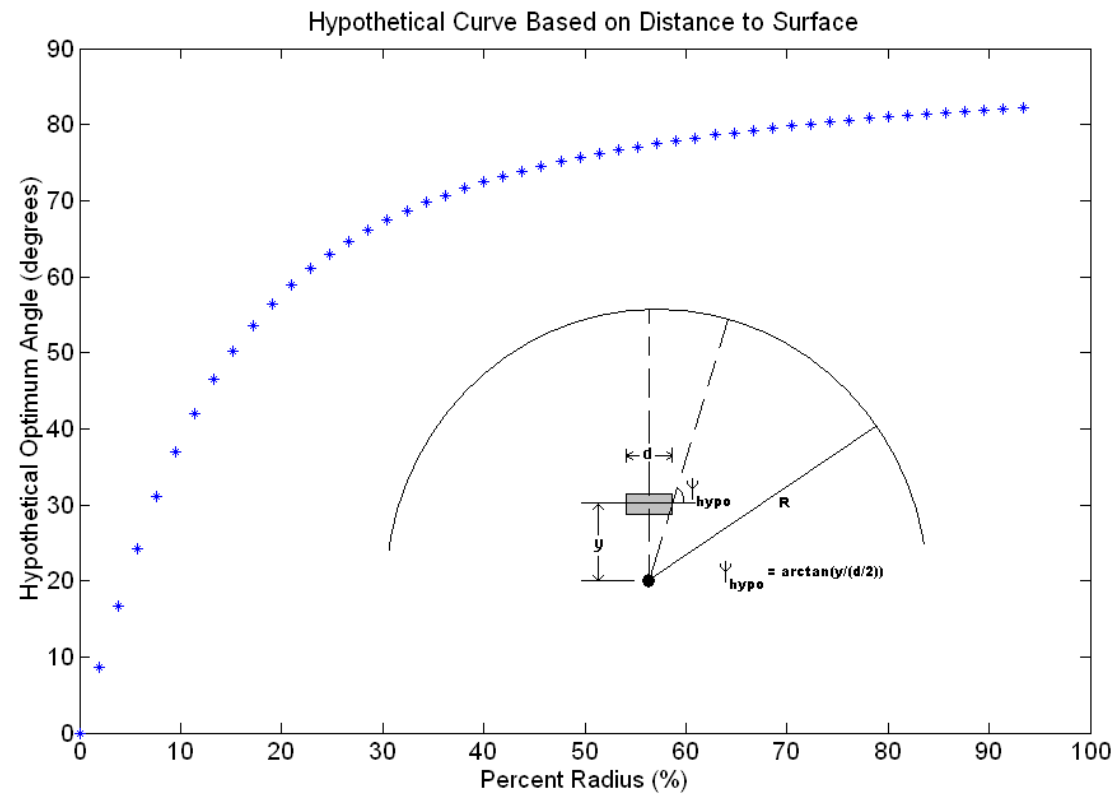

Figure 14: As the antenna moves towards the surface, the optimum angle increases and is concave down. This graph is based on (3.1) and code is found in Appendix .9. 

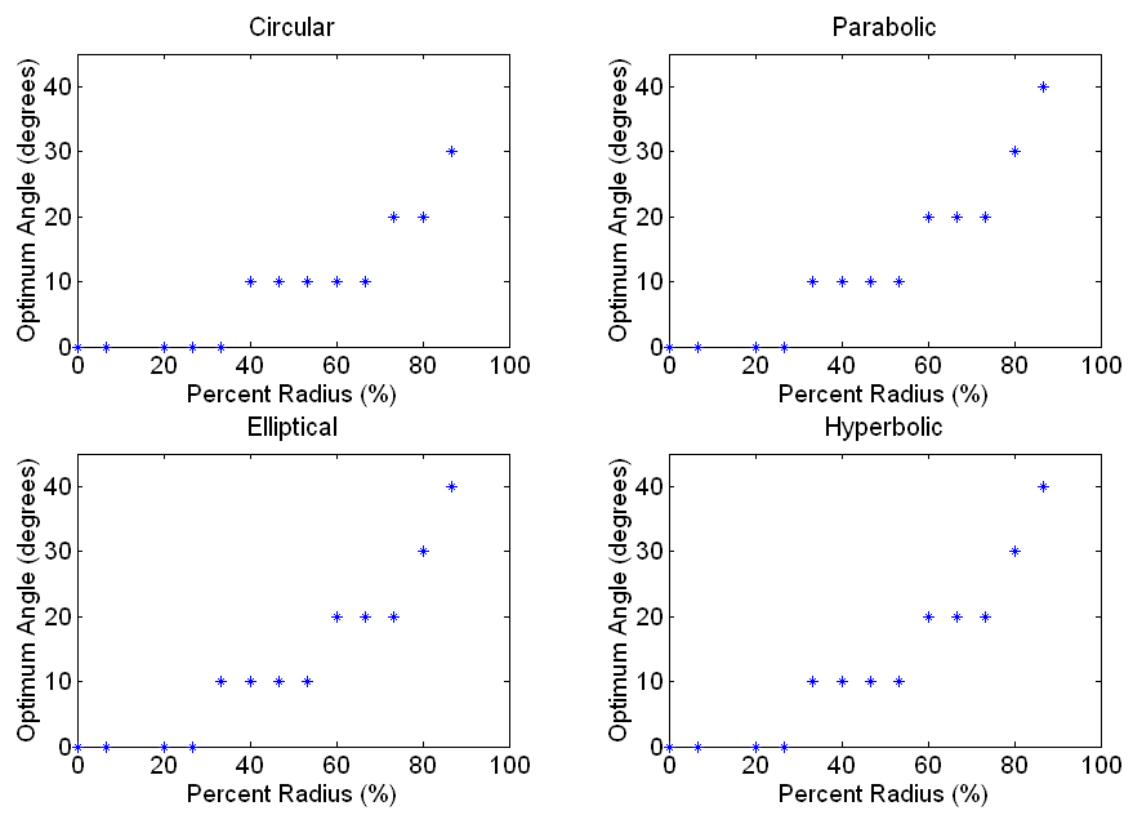

Figure 15: Shows that as the antenna is moved toward the surface, the optimum angle rotates so that the antenna elements will point towards the surface (Appendix .9).

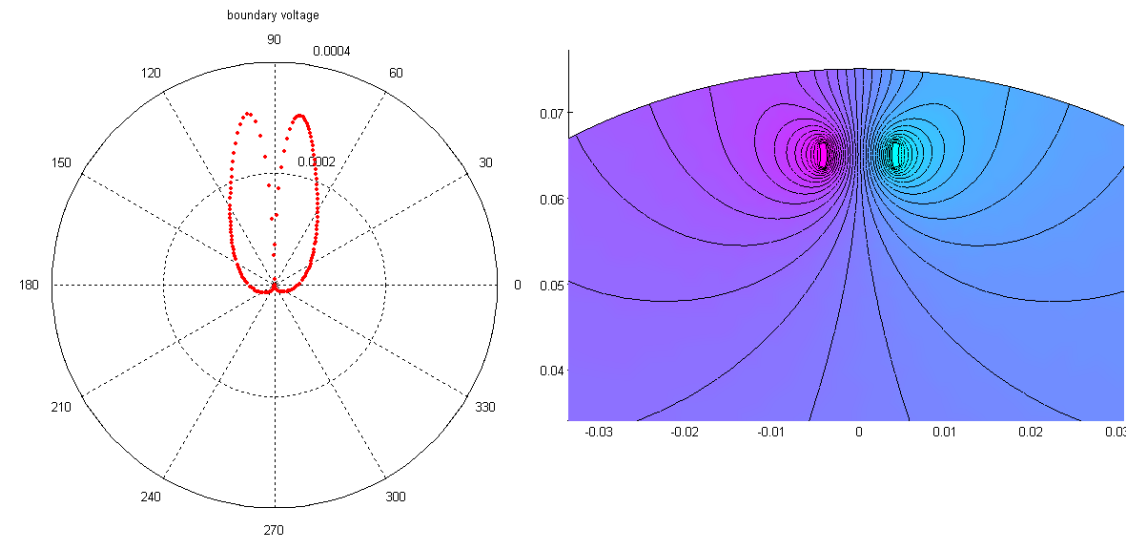

Figure 16: The antenna elements are simulated without an insulator between the elements. The polar plot displays the surface voltage. The right plot shows equipotential lines surrounding the antenna elements. The polar plot has units of Volts (Appendix .12). 

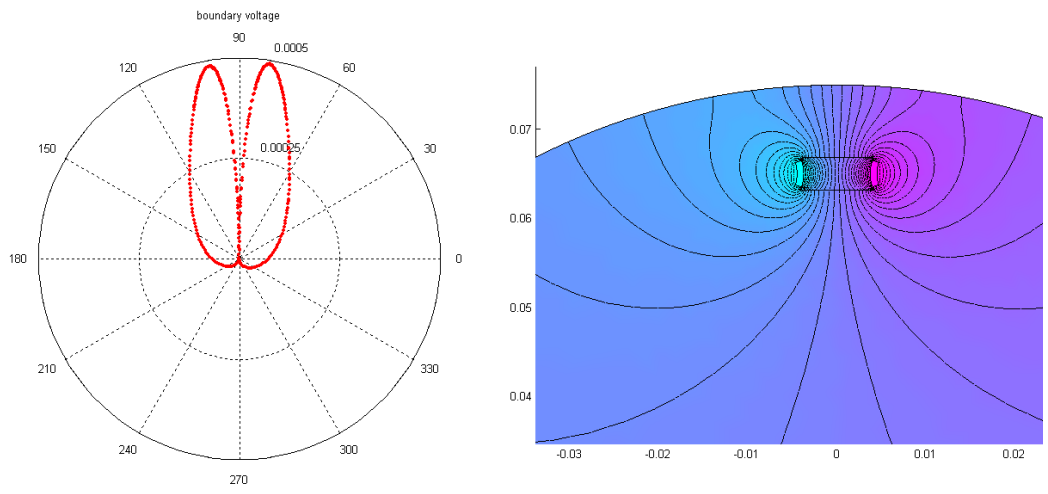

Figure 17: The antenna elements are simulated only with an insulator between the elements. The polar plot displays the surface voltage. The right plot shows equipotential lines surrounding the antenna elements. The polar plot has units of Volts (Appendix .11).
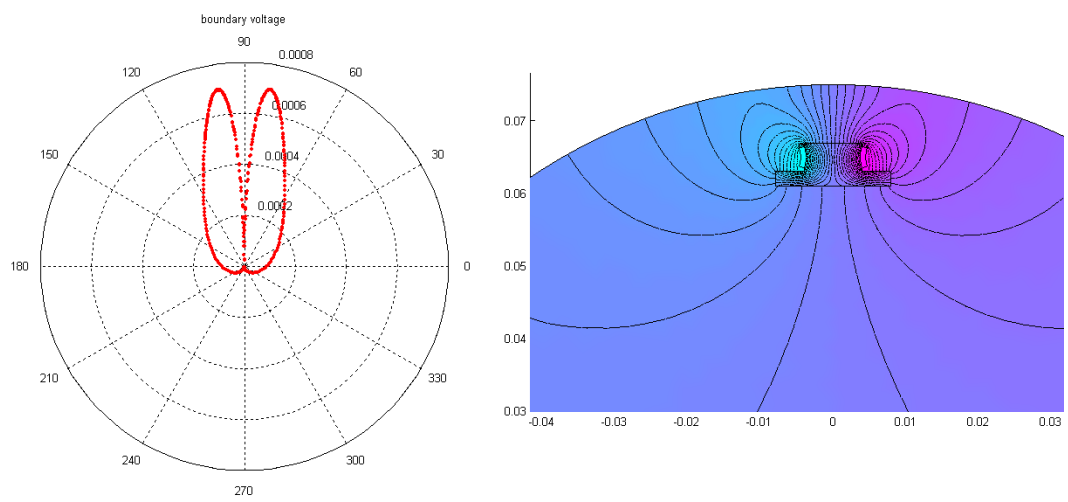

Figure 18: An insulating material is placed below the antenna elements to act as a current reflector. The polar plot displays the surface voltage. The right plot shows equipotential lines surrounding the antenna elements. The polar plot has units of Volts (Appendix .13). 
Table 3: Results for Proofs of Concept

\begin{tabular}{|c|c|}
\hline Analysis & Max. Surface Voltage $(\mathrm{mV})$ \\
\hline No Epoxy (2D) & 0.3 \\
\hline Epoxy (2D) & 0.5 \\
\hline Reflector (2D) & 0.7 \\
\hline No Epoxy (3D) & 12 \\
\hline Epoxy (3D) & 16 \\
\hline
\end{tabular}

\subsubsection{Proof of Concept in $3 \mathrm{D}$}

Again, to show proof of concept that the far field can be affected by near field changes, a 3D numerical analysis was done comparing:

1. Antenna elements without epoxy (Figures 20 and 21).

2. Antenna elements with epoxy (Figures 22 and 23 ).

For the three-dimensional analysis, two slices were taken. These slices are shown in Figure 19. Again, as in the 2D case, note that the absolute value of the voltage was taken so that polar plots could be made. It is clear that the simulations with epoxy between the elements have higher surface voltages. Although no 3D simulations were done with a reflector, from the given $2 \mathrm{D}$ and $3 \mathrm{D}$ results, it is evident that one can use an insulator between the elements to increase the surface voltage. Table 3 compiles the results for the proofs of concept.

\subsubsection{Finite Element Analysis in 3D}

The results of the 3D FEA are shown in Figure 24. These results incorporate the epoxy into the simulation. Fewer points were simulated due to the large amount of time and user input required to carry out the simulation. Although the shape of these results is much like Figure 13, the FE results are approximately 30-35 percent larger than the analytical results. 


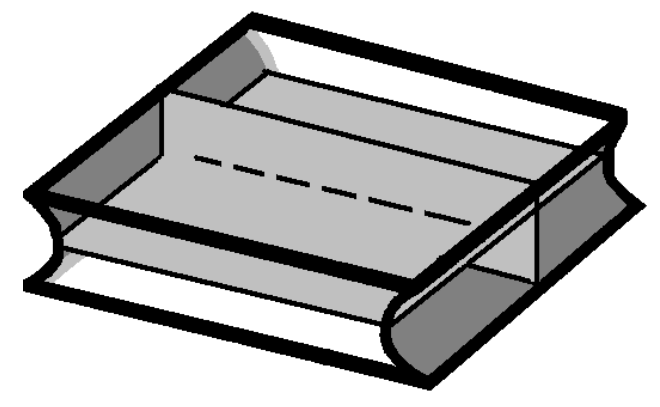

Figure 19: Depicts the 2 planes where the slices were taken.
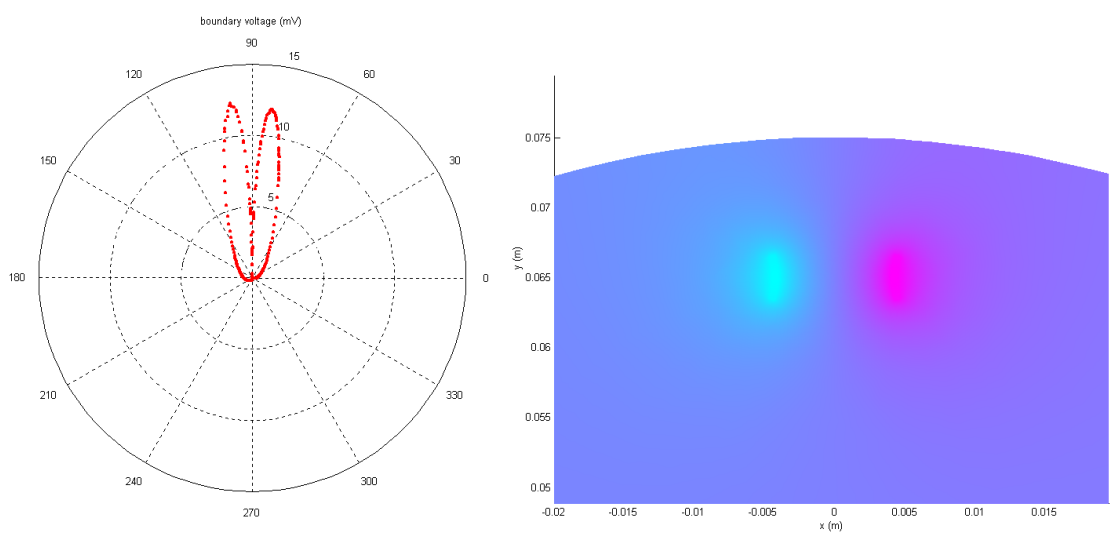

Figure 20: The antenna elements are simulated without an insulator between the elements. The polar plot displays the surface voltage (Code to extract slices from FEMLab in Appendix $.10)$. 

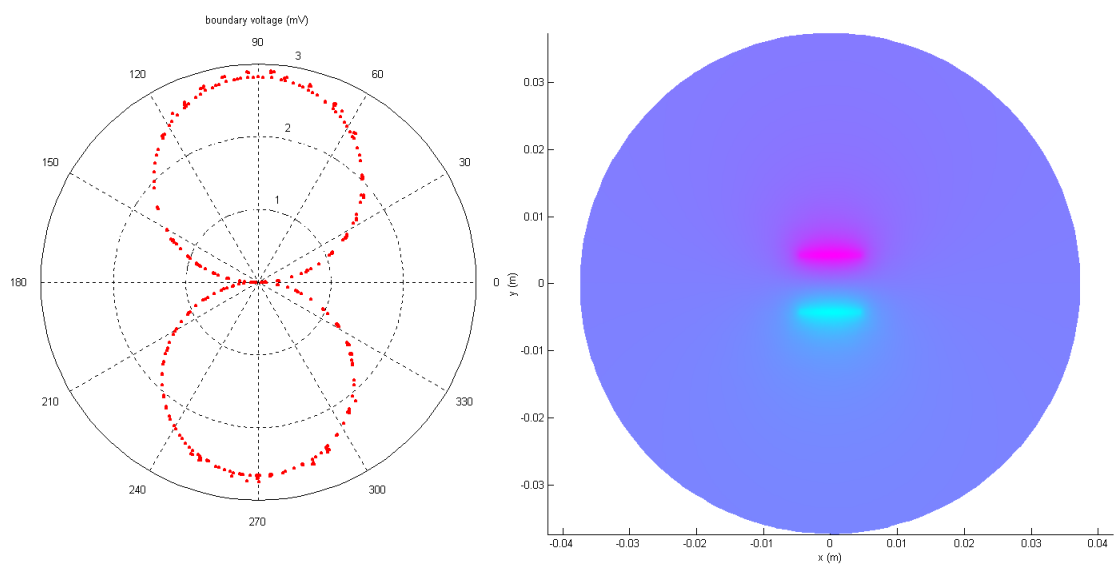

Figure 21: The antenna elements are simulated without an insulator between the elements. The polar plot displays the surface voltage.
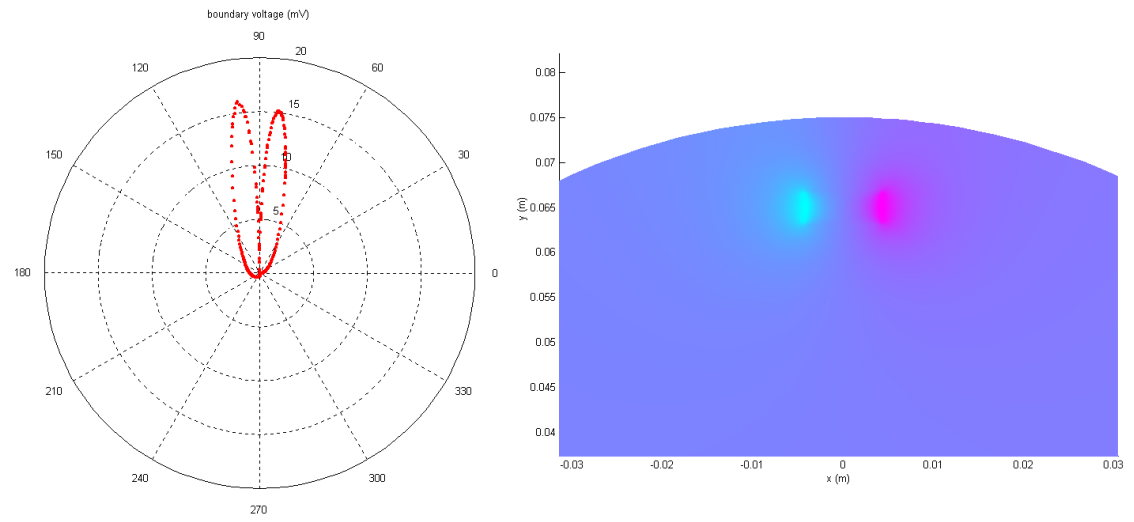

Figure 22: The antenna elements are simulated with an insulator between the elements. The polar plot displays the surface voltage. 

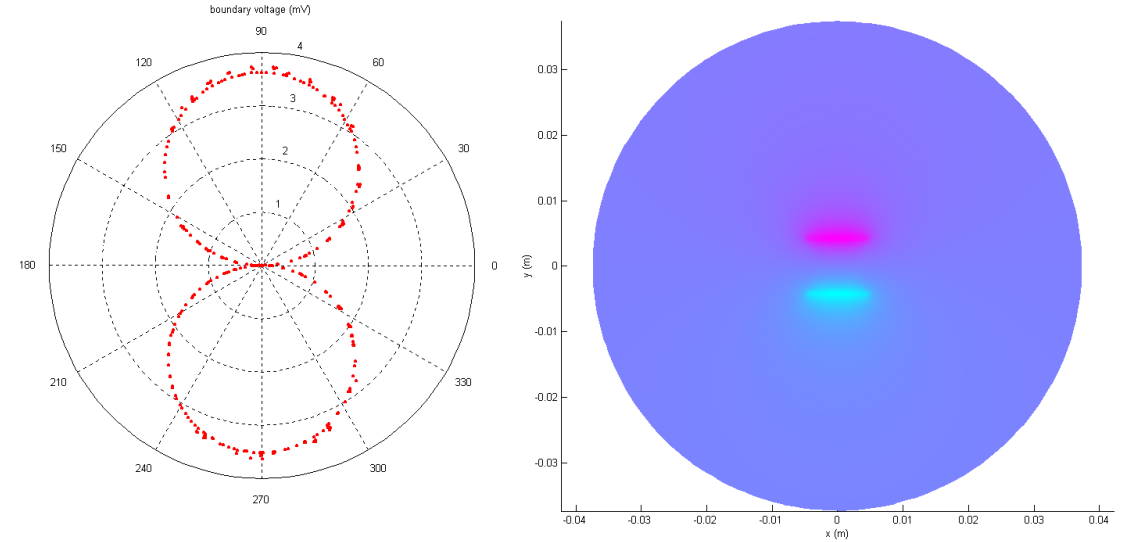

Figure 23: The antenna elements are simulated with an insulator between the elements. The polar plot displays the surface voltage.
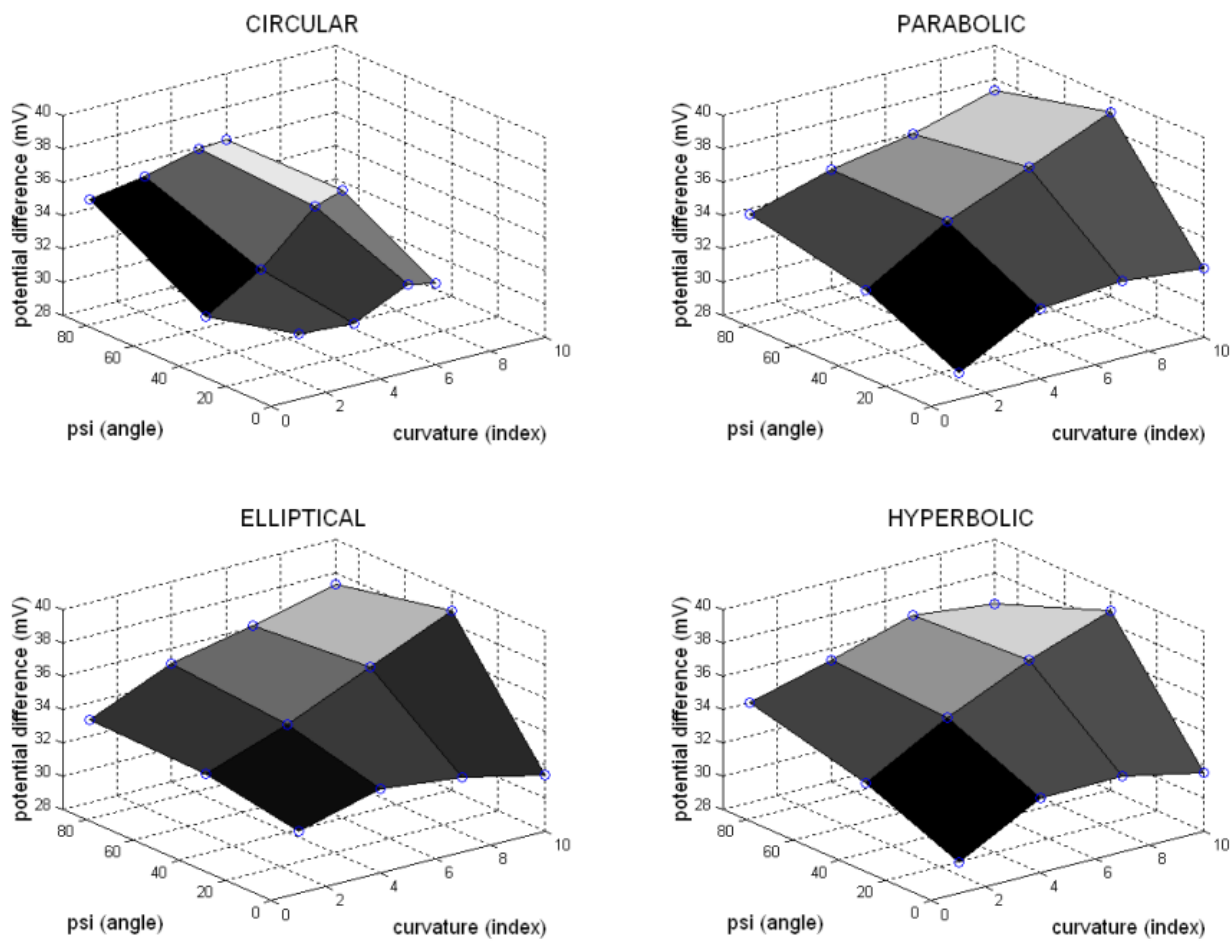

Figure 24: Results for the FEA with epoxy between the antenna elements. Top left: Semicircular Top right: Parabolical Bottom left: Elliptical Bottom right: Hyperbolic 


\subsection{DISCUSSION}

Several issues will be explored in the discussion. The first will be the verification of the finite element results by creating similar geometries and source conditions in the analytical domain. Second will be the parameters used in creating the finite element results. Specifi-

cally, convergence of the solution will be tested for increasing difference between the epoxy and antenna element conductivities. Ideally, the different conductivities could be explicitly expressed in the FEA but numerical instability does not allow this. Thus, as long as the conductivities are sufficiently different, the solution should not vary to a significant degree.

\subsection{VERIFYING ANALYTICAL AND NUMERICAL RESULTS FOR THE 1-SHELL MODEL}

For comparability, it is important that similar geometries give similar results for the analytical and numerical cases. Further, it also proves that the FEA is being set up correctly. To this end, FE models were made which were similar to closed-form models already created. The following models were compared:

1. Nonideal dipole around the center of a sphere (Figure 25).

2. Nonideal dipole near the surface of a sphere (Figure 26).

3. Antenna elements at the surface (Figure 27).

4. All shapes, curvatures and angles (Figure 28).

From Figures 25-27 one can see that the magnitudes are the same for all three types of simulated shapes. For these cases it was easy to recreate the geometries from the analytical model to the $\mathrm{FE}$ one, however this proved to be a more difficult task for the fourth case, which 

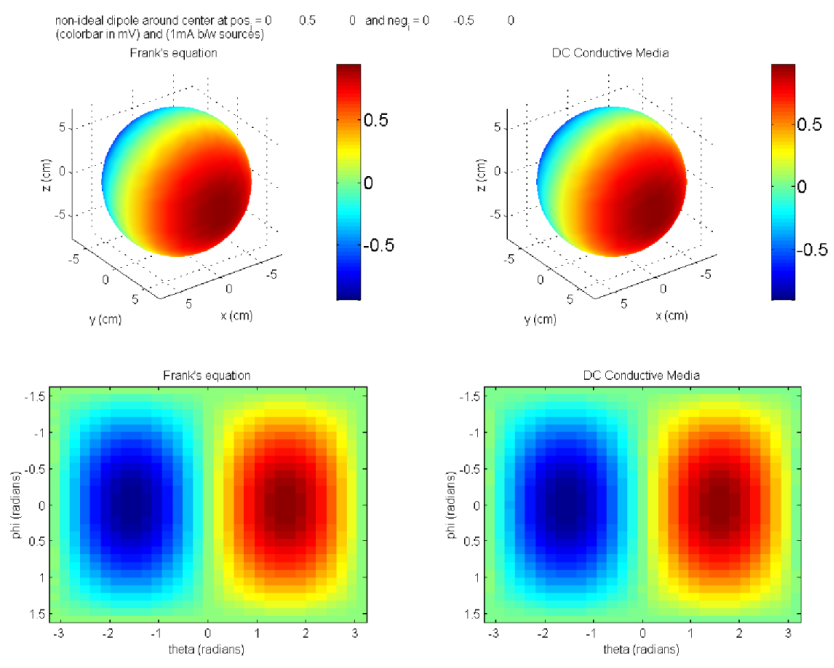

Figure 25: Comparison of Finite Element and Frank equation solution for (nonideal dipoles) current sources around the center. The top plots show the potential plotted on the surface of a spherical model of the head. The bottom plots show the same voltage distribution plotted on a grid (Appendix .5).

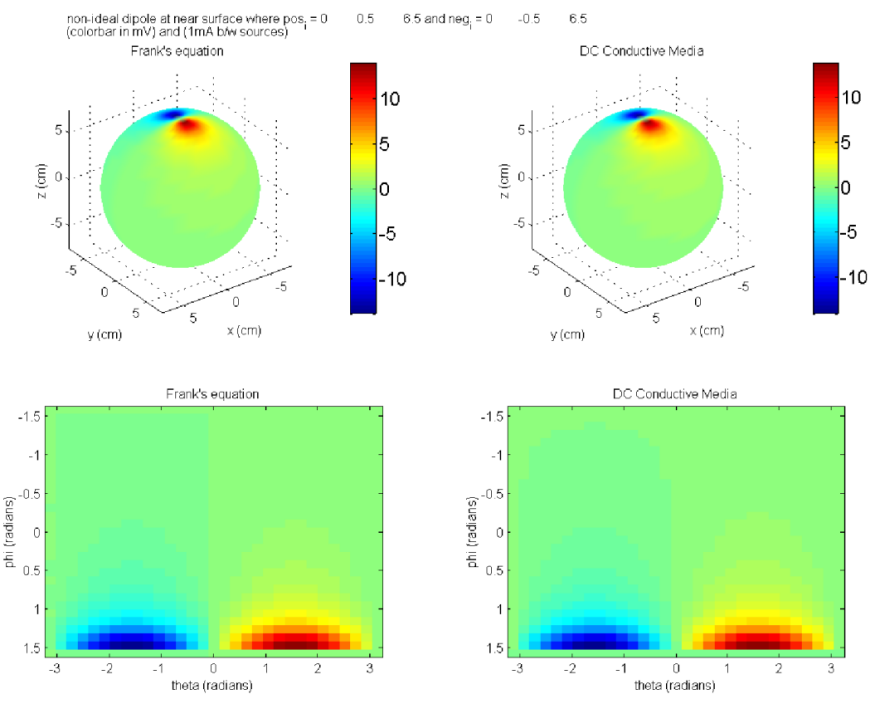

Figure 26: Comparison of FEM and Frank equation solution for current sources near the surface. The top plots show the potential plotted on the surface of a spherical model of the head. The bottom plots show the same voltage distribution plotted on a grid. 

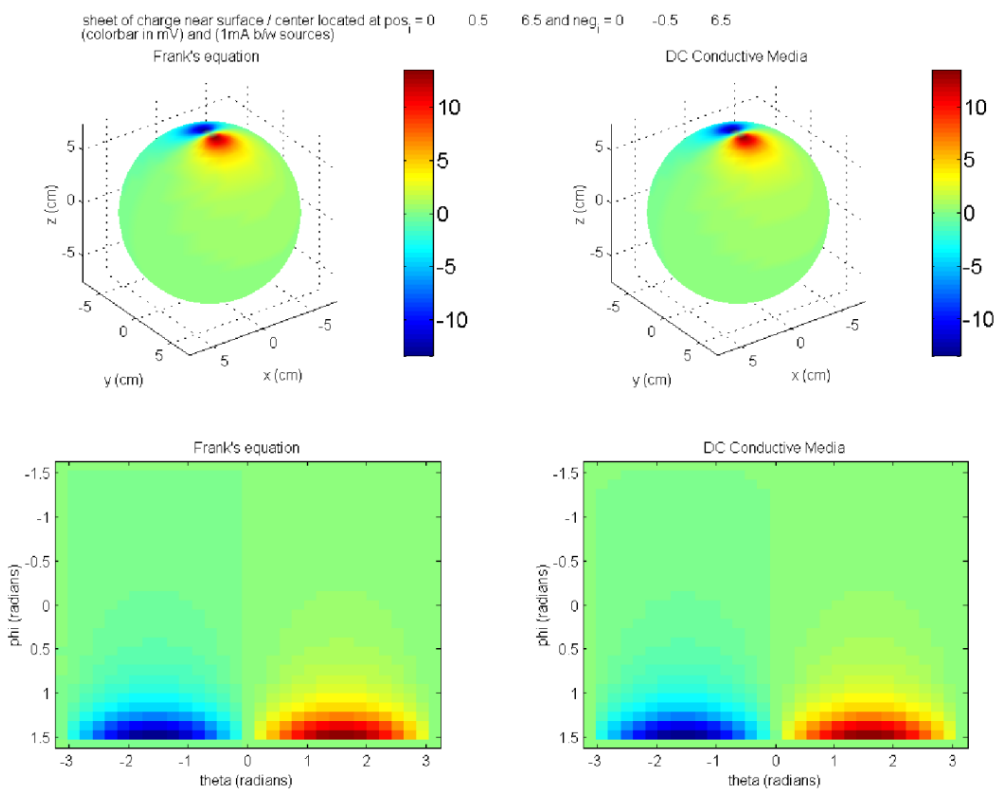

Figure 27: Comparison of FEM and Frank equation solution for current sheets near the surface.
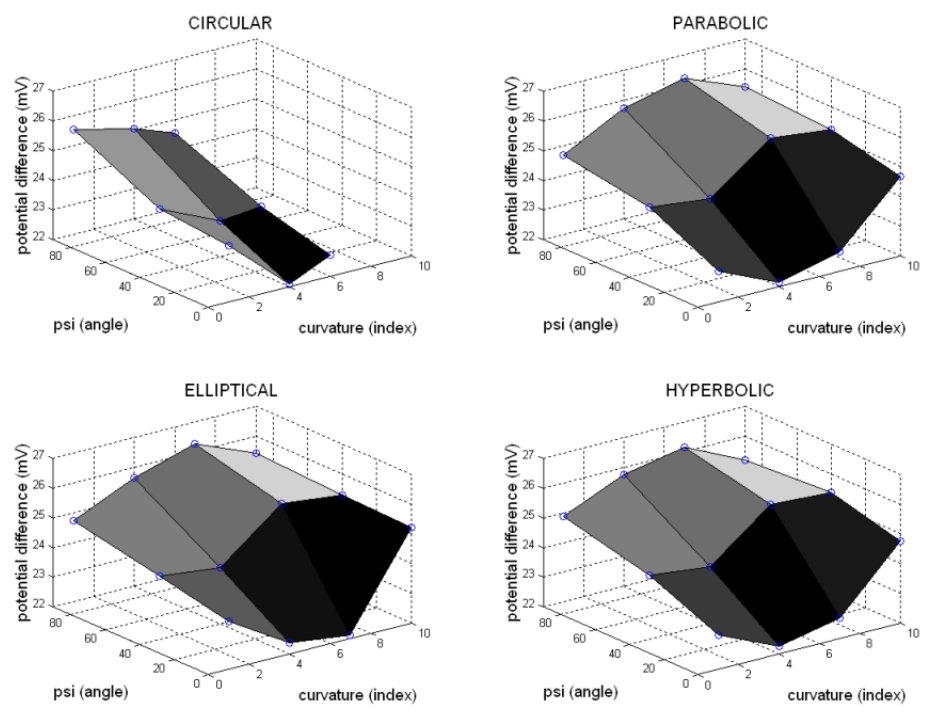

Figure 28: Finite element solution for all shapes, angles and curvatures where there is no epoxy between the elements. These results should compare well with Figure 13. The error is shown in Figure 29 (Code found in Appendix .16). 

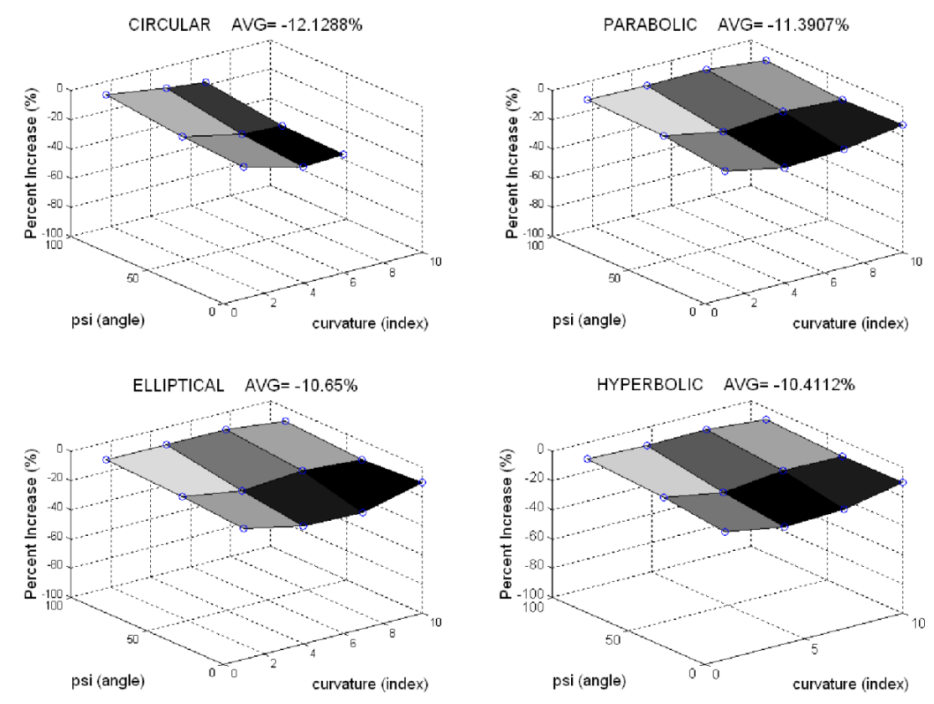

Figure 29: Comparison of FEM and Frank equation solution for all shapes, angles and curvatures. The percent increase represents how different the finite element solution is compared to the analytical.

is shown Figure 28. In the analytical model the antenna elements were modeled as sheets and thus had an infinitesimal thickness. This cannot be done in a FE domain because if the antenna element thickness was made too small, the solution did not converge. Therefore, Figure 29 describes this error. The typical error value is about $-11 \%$, meaning the FE results were lower in voltage. The percent error was calculated by the following equation:

$$
\% \text { error }=\frac{\text { FiniteElement }- \text { Analytical }}{\text { Analytical }} * 100
$$

\subsubsection{Comparing FE with Epoxy to Analytical Solutions}

Now that it has been established that the analytical can be compared to the FEM, it is important to see how simulating the epoxy alters the result. Therefore, the percent increase can be calculated using the results from (4.1) and Figures 13 and 24. Therefore, one can see that the epoxy increases the surface voltage between 30 and 35 percent. 

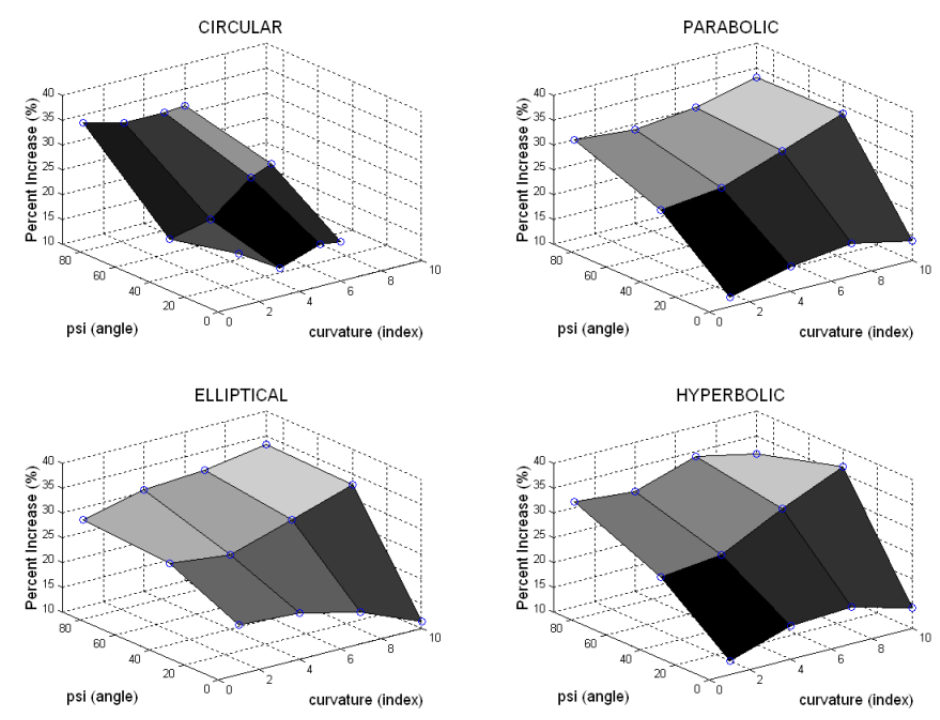

Figure 30: Comparison of FEM and Frank equation solution for all shapes, angles and curvatures with epoxy. The percent increase represents how the epoxy does not allow current to short between the antenna elements and thus forces it to go around the antenna and give better surface voltages (Appendix .15). 

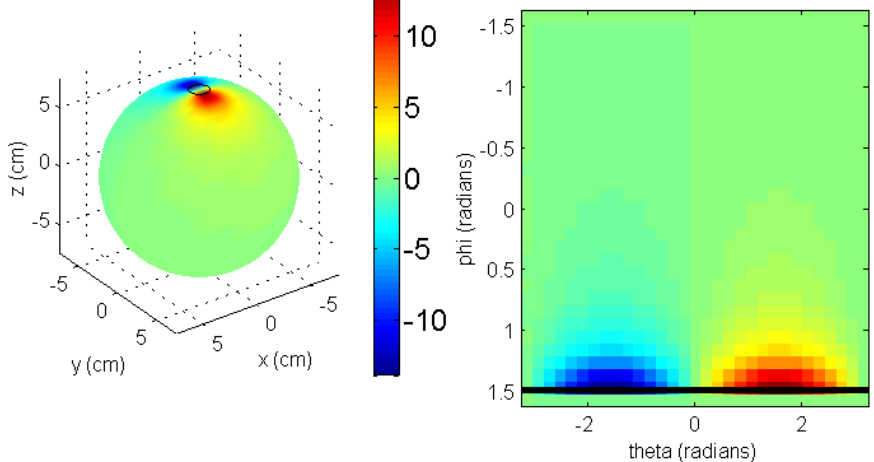

Figure 31: The black line shows the maximum value for each column which is confined to only one row or in other words, one value of angle $\phi$ (Appendix .5).

\subsubsection{Basis Functions}

Originally, the supplied volume for the current source and sink had been incorrectly specified. This caused the volume current density to be underestimated. This error was quantified by taking the maximum values for each column of the $\phi$ vs $\theta$ array. Because of the symmetry of the dipoles, this turned out simply to be one row i.e., a constant value of $\phi$. This can be seen in Figure 31.

After finding the maximum values from the Frank equation (2.28) and FEA, they were subtracted to find the error which resulted in the top plot of Figure 32.

From the bottom plot of Figure 32 it is easy to assume that the error is a sinusoid. At first it was thought a mistake had been made because the error was sinusoidal, but it was soon realized that if both plots were sinusoids with different amplitudes of the same frequency then their difference (error) is also a sinusoid. Thus, it was found that at the maximum, the voltage is sinusoidal. But was this true across all constant values of $\phi$ ? For insight the voltage was plotted for all values of $\phi$ and visually inspected. Figure 33 shows all rows together from several views. These are indeed all sinusoids of varying amplitude.

Thus, for this configuration of the current source the amplitude of the voltage on the surface of the sphere is simply a modulated sine function. It would be interesting to know why this occurs so a closed-form equation for an ideal dipole was manipulated to see how 

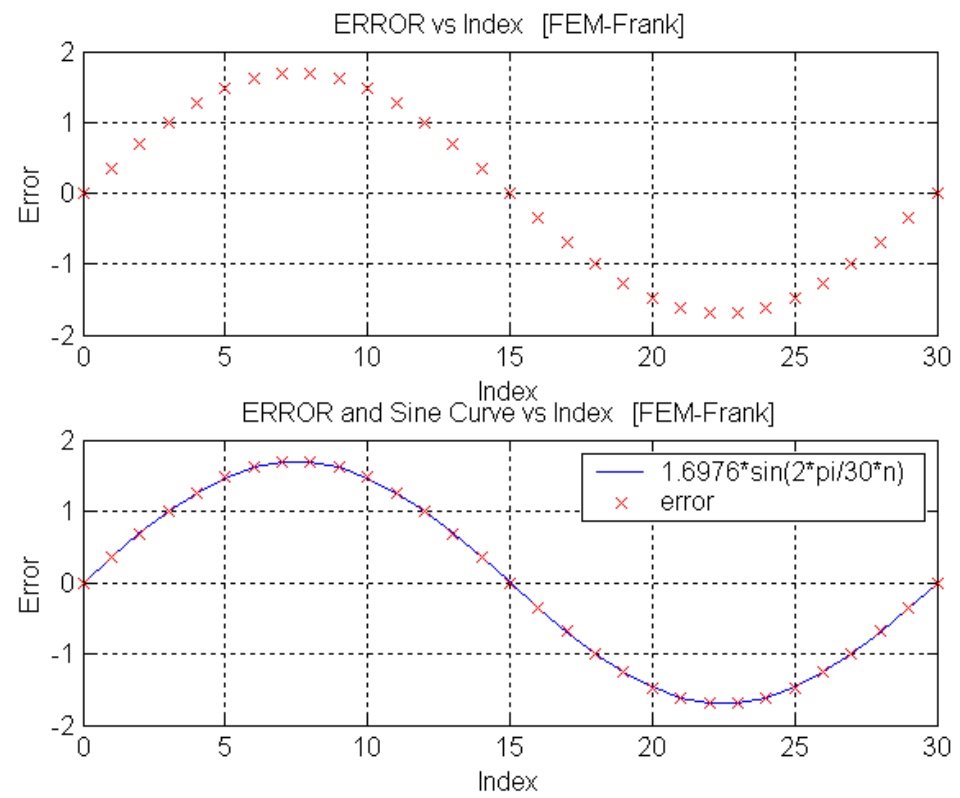

Figure 32: Top Plot: Error. Bottom Plot: Error with a sine curve overlaid (Appendix .5).

the sine curve emerges. The equation for a unit-radius spherical volume conductor with a homogeneous conductivity and an ideal dipole inside has the form in (2.27). The dipole whose surface voltage had been found had the following simplifications:

$\vec{m}=\left(0, m_{y}, 0\right)$ i.e., the dipole moment is in (or opposite to) the direction of the y-axis

$\vec{d}=\left(0,0, d_{z}\right)$ i.e., the dipole was placed on the z-axis

$\vec{e}=(\cos \phi \cos \theta, \cos \phi \sin \theta, \sin \phi)$

$\mathrm{q}=|\mathrm{e}-\mathrm{d}|=\left|\left(\cos \phi \cos \theta, \cos \phi \sin \theta, \sin \phi-d_{z}\right)\right|$

$$
=\left(1-2 d_{z} \sin \phi+d_{z}^{2}\right)^{1 / 2}
$$

$\mathrm{s}=\mathrm{e} \cdot \mathrm{d}=d_{z} \sin \phi$

Plugging this back into the equation we get 

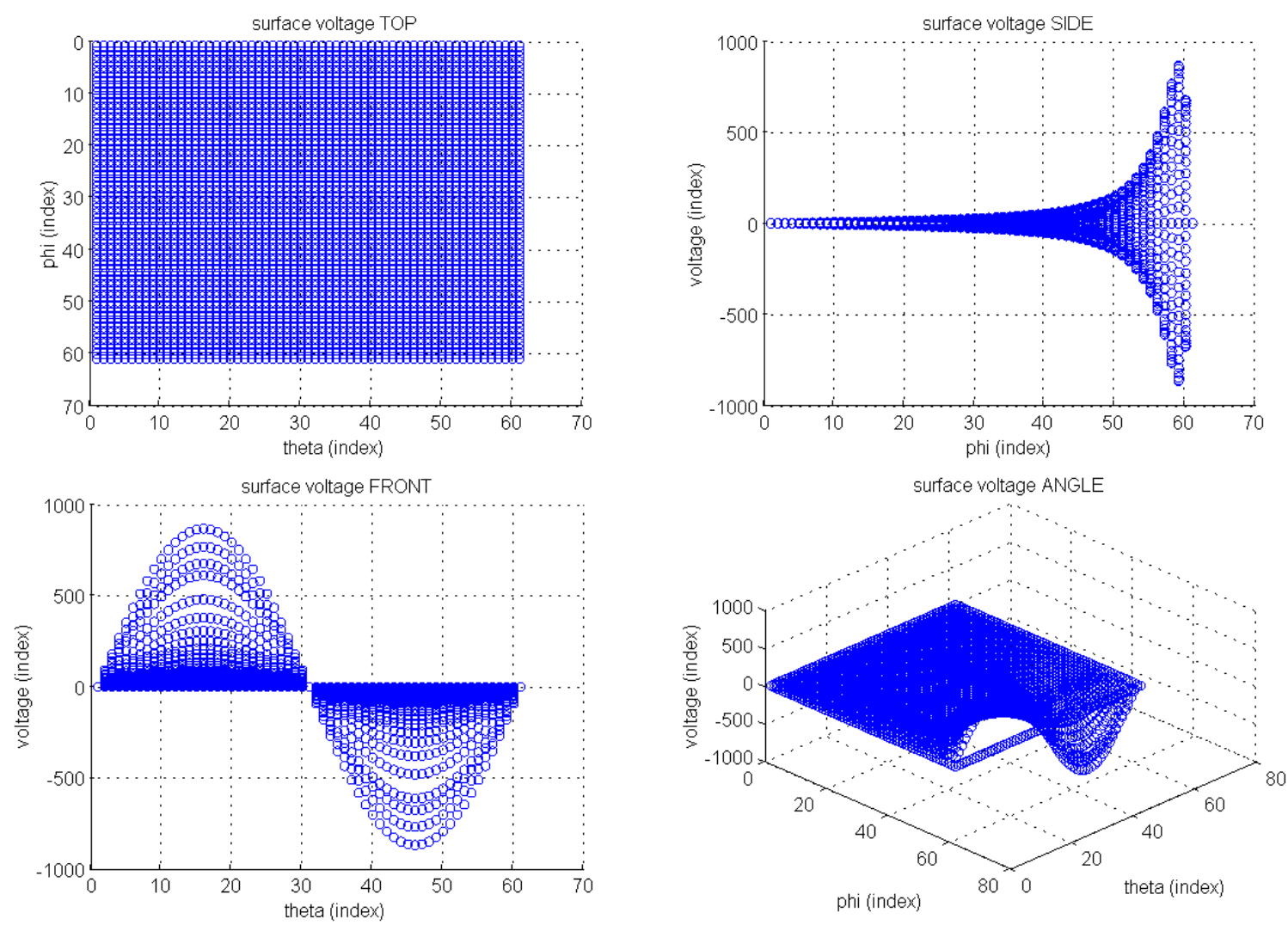

Figure 33: Different angles of the voltages plotted on a rectangular grid (Appendix .5). 


$$
\begin{aligned}
& \Phi=\frac{1}{4 \pi \sigma q} m_{y}\left\{\frac{2 \cos \phi \sin \theta}{\left(1-2 d_{z} \sin \phi+d_{z}{ }^{2}\right)^{3 / 2}}+\frac{\cos \phi \sin \theta}{\left(1-2 d_{z} \sin \phi+d_{z}{ }^{2}\right)^{1 / 2}}+\right. \\
& \left.\frac{\cos \phi \sin \theta \sin \phi d_{z}}{\left(1-2 d_{z} \sin \phi+d_{z}{ }^{2}\right)^{1 / 2}\left[\left(1-2 d_{z} \sin \phi+d_{z}\right)^{1 / 2}+1-d_{z} \sin \phi\right]}\right\}
\end{aligned} .
$$

Pulling out the $\sin \theta$ term:

$$
\begin{aligned}
\Phi= & \frac{1}{4 \pi \sigma q} m_{y} \sin \theta\left\{\frac{2 \cos \phi}{\left(1-2 d_{z} \sin \phi+d_{z}{ }^{2}\right)^{3 / 2}}+\frac{\cos \phi}{\left(1-2 d_{z} \sin \phi+d_{z}{ }^{2}\right)^{1 / 2}}+\right. \\
& \left.\frac{\cos \phi \sin \phi d_{z}}{\left(1-2 d_{z} \sin \phi+d_{z}\right)^{1 / 2}\left[\left(1-2 d_{z} \sin \phi+d_{z}{ }^{2}\right)^{1 / 2}+1-d_{z} \sin \phi\right]}\right\}
\end{aligned} .
$$

From the equation above, one can see that when $\phi$ is constant, the voltage $\Phi$ is a sine wave that is only a function of $\theta$. So we can break $\Phi$ into two parts:

$$
\Phi=\Phi_{\theta} \Phi_{\phi} \quad
$$

where,

$$
\Phi_{\theta}=\sin \theta \quad,
$$

and

$$
\begin{gathered}
\Phi_{\phi}=\frac{1}{4 \pi \sigma q} m_{y}\left\{\frac{2 \cos \phi}{\left(1-2 d_{z} \sin \phi+d_{z}^{2}\right)^{3 / 2}}+\frac{\cos \phi}{\left(1-2 d_{z} \sin \phi+d_{z}^{2}\right)^{1 / 2}}+\right. \\
\left.\frac{\cos \phi \sin \phi d_{z}}{\left(1-2 d_{z} \sin \phi+d_{z}\right)^{1 / 2}\left[\left(1-2 d_{z} \sin \phi+d_{z}\right)^{1 / 2}+1-d_{z} \sin \phi\right]}\right\}
\end{gathered} .
$$

Plugging $q$ and s back in:

$$
\Phi_{\theta}=\sin \theta \quad,
$$

and

$$
\Phi_{\phi}=\cos \phi\left\{\frac{1}{4 \pi \sigma} m_{y}\left[\frac{2}{q(\phi)^{3}}+\frac{1}{q(\phi)}+\frac{s}{q(\phi)(q(\phi)+1-s)}\right]\right\} \quad .
$$


Thus one can see more clearly that $\Phi_{\theta}$ is modulated by $\Phi_{\phi}$. These equations may be useful to better interpolate between known potentials gathered from the head. Following that the voltage on the head is created by a superposition of many current dipoles in the head, these two equations could be used to reduce the possible number of ways in which one can intelligently interpolate between measured voltages. Similar methods exist for MEG analysis [20].

\subsection{SAMPLING THE SURFACE OF THE SHAPES}

It is important to note that 100 monopoles per curve were evenly placed according to the arc length. This spacing was also used for the long axis. This is a measure of the density of sources and sinks used. As previously mentioned, it is important that enough sources and sinks be placed on the surface of the antenna elements so that the shape is captured. The values for 40 and 10 monopoles per curve were also calculated. The max deviation was 0.05 and $0.25 \mathrm{mV}$, respectively. This corresponds to a percent error of 0.23 and 1.1 percent. Thus, it does not seem necessary to have 100 monopoles per curve which is much more time consuming than the lower values.

\subsection{CONVERGENCE OF THE SOLUTION VS. CONDUCTIVITY DIFFERENCE}

This problem arose due to the disparity in conductivity values of the antenna elements and the epoxy but only needed to be addressed for the 3D analysis. From Table 1 it is evident that

these conductivity $\left(\frac{1}{\text { Resistivity }}\right)$ values are different by 20 orders of magnitude. When these two materials are juxtaposed together in the simulation it can cause numerical problems. Therefore, a graph was made as can be seen in Figure 34 that shows the point at which at which the solution converges. The curve seems to level off at a value of 4 . This means that the antenna element conductivity should be ten thousand times larger than the epoxy conductivity. These values are centered around the sphere conducitivity. Therefore, to obtain 


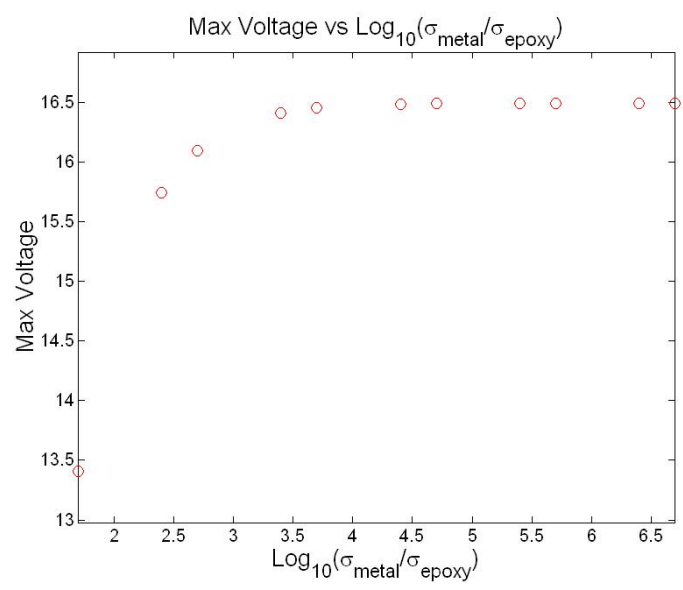

Figure 34: Graph shows that for an increasing conductivity difference, the solution does not change (Appendix .17) .

the antenna element conductivity, the sphere conductivity was multiplied by $\sqrt{10,000}$. To obtain the epoxy conductivity, the sphere conductivity was divided by $\sqrt{10,000}$. Thus, the antenna element conductivity is 10,000 times larger than the epoxy conductivity.

\subsection{IMPEDANCE}

From Figures 35 and 36 one can see that the average voltages required by the antenna are about 70 and $120 \mathrm{mV}$. For a constant current of $1 \mathrm{~mA}$, the distributed resistance seen by the antenna is about 140 and $240 \Omega$. These will be important parameters to be verified experimentally. Further, one will have to take these values into account when designing the power source and ciruit to drive signal transmission. It is also important to note that as the curvature is increased, the required source voltage increases. This is because as the antennas close (increase curvature) the current is forced through a smaller area. Therefore, to maintain a constant current of $1 \mathrm{~mA}$, the voltage must increase accordingly. 

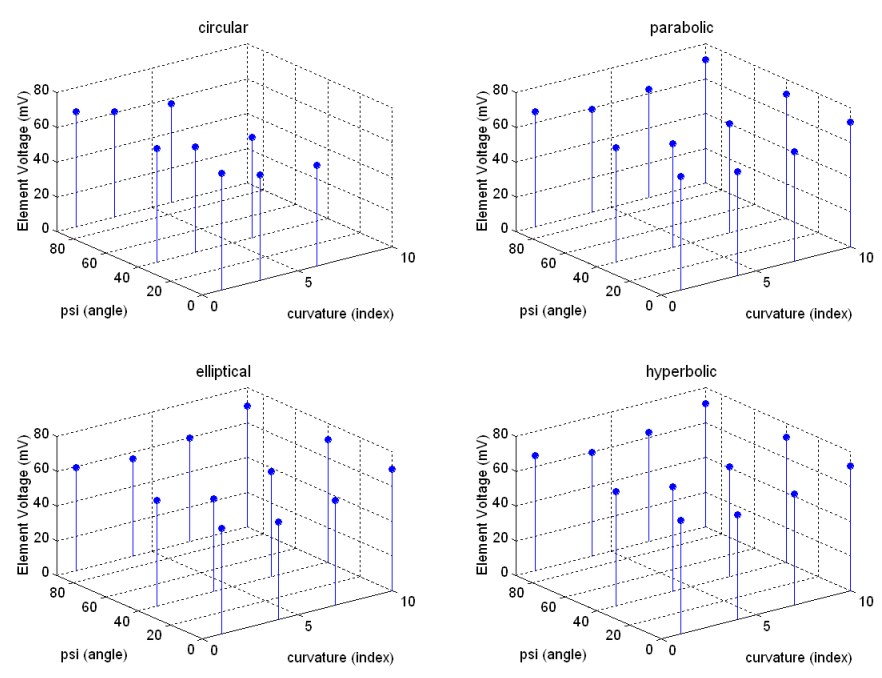

Figure 35: Maximum element voltage plotted versus angle and curvature with no epoxy between the antenna.
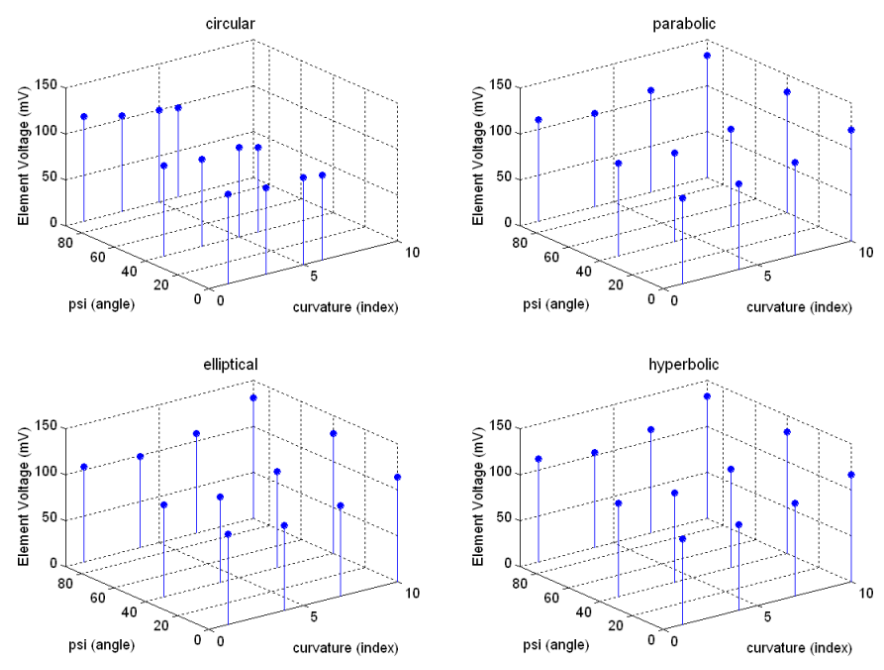

Figure 36: Maximum element voltage plotted versus angle and curvature with epoxy between the antenna. 


\subsection{CONCLUSIONS}

In conclusion, two- and three-dimensional finite element analyses show proof of concept that changes to the near field geometry and properties of the antenna can alter the far field results. It was determined that the correct model to analytically simulate the volume conduction antenna was a nonideal dipole. It was also established that the analytical and numerical results were in agreement for similar geometries, therefore the results could be compared and appropriate conclusions drawn when performance changed. The analytical and numerical analysis showed that building an antenna pointed at the surface but slightly away from the other antenna and having large curvature results in the most efficient signal transmission. Numerical results agree but are capable of simulating the antenna more realistically by integrating the epoxy into the analysis. This more realistic analysis yielded a $30-35 \%$ increase in surface potential. 


\section{APPENDIX}

MATLAB AND MEX CODE

\section{.1 GRAPHICAL USER INTERFACE}

Contains the following files:

- shapescreator.m

- shapescreator2d.m

- shapesfun.m

- shapesfundersq.m

- shapesgui.m

- shapesnext.m

- shapesnumerical.m

- shapesplt3d.m

- frankpotential.c

- potential.c 


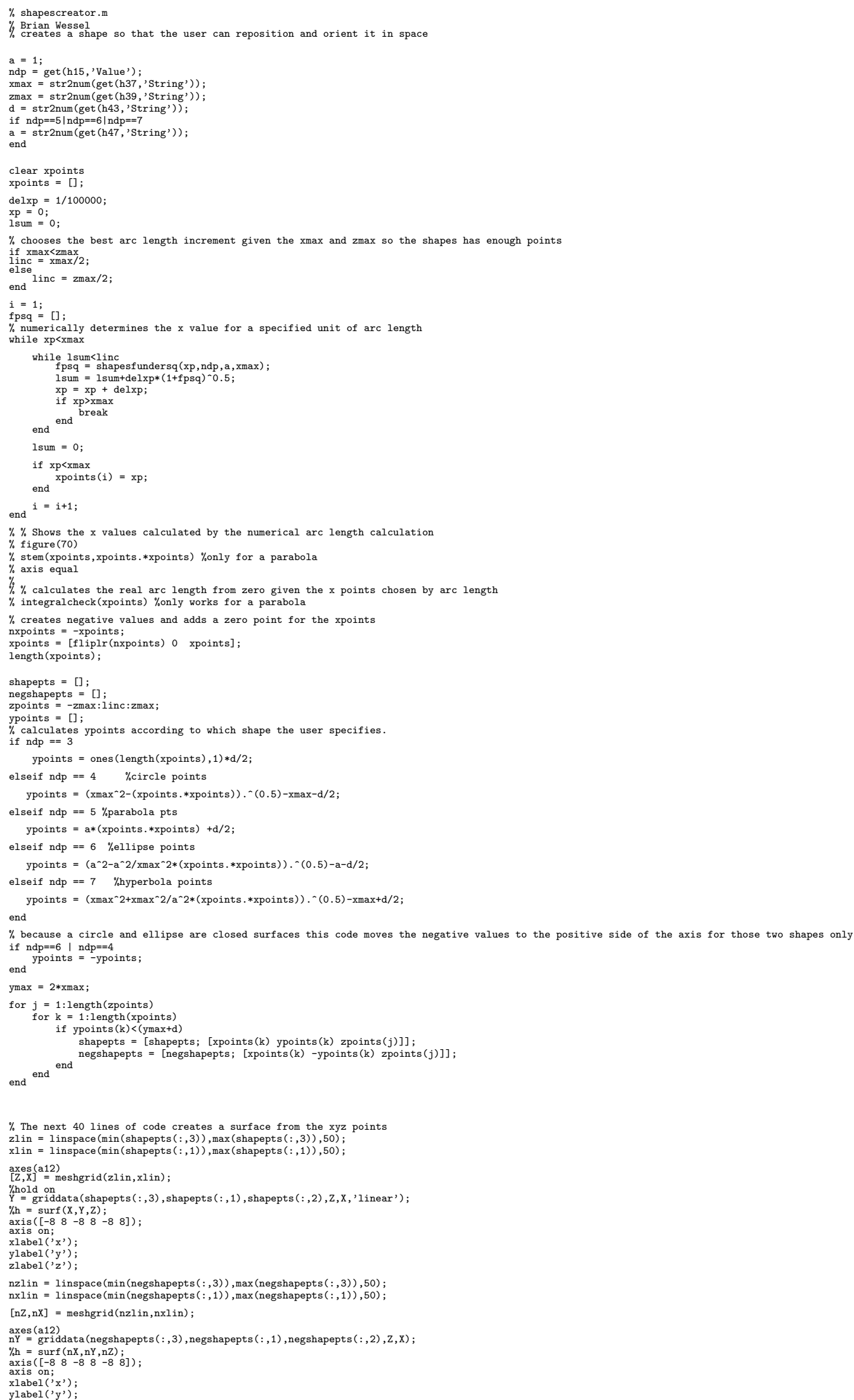


zlabel(' $z$ ');

$\mathrm{XX}=[\mathrm{X} ; \mathrm{nX}] ;$

$\mathrm{ZZ}=[\mathrm{Z} ; \mathrm{nZ}]$

$\mathrm{h}=\operatorname{surf}(\mathrm{XX}, \mathrm{YY}, \mathrm{ZZ})$;
shading interp

axis $\left.\left(\begin{array}{llllll}-8 & 8 & -8 & 8 & -8 & 8\end{array}\right]\right)$

xlabel(' $x$ ');

ylabel( $y$ ');

zlabel(' $z$ '); 


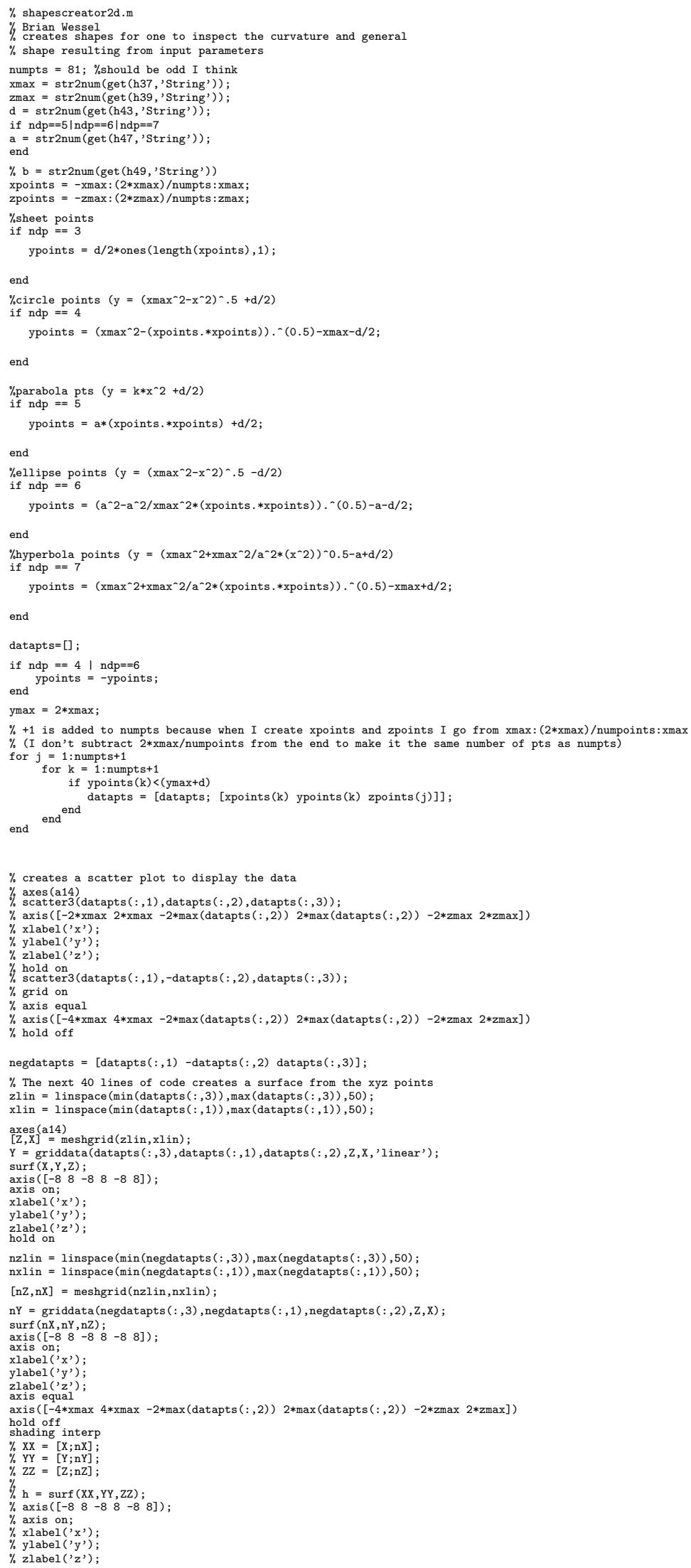


$\%$ shapesfun.m

$\%$ outputs a point for input parameters

function $f=\operatorname{shapesfun}(x x, n, a, x \max , d)$

if $\mathrm{n}==3 \%$ sheet points

$f=d / 2$;

elseif $n=4, \%$ circle points $\left(y=\left(\max ^{\wedge} 2-x^{\wedge} 2\right)^{\wedge} .5+d / 2\right)$

$f=(x \max \wedge 2-(x x \cdot * x x)))^{\wedge}(0.5)-x \max -d / 2 ;$

elseif $\mathrm{n}==5 \%$ parabola pts $\left(\mathrm{y}=\mathrm{k}^{*} \mathrm{x}^{\wedge} 2+\mathrm{d} / 2\right) \mathrm{n}==5$,

$f=a *(x x \cdot * x x)+d / 2 ;$

elseif $n==6, \quad \%$ ellipse points $\left(y=\left(x_{\max }^{\wedge} 2-x^{\wedge} 2\right)^{-} .5-d / 2\right)$

$f=\left(a^{\wedge} 2-a^{\wedge} 2 / x \max \wedge 2 *(x x \cdot * x x)\right) \cdot{ }^{\wedge}(0.5)-a-d / 2 ;$

elseif $\mathrm{n}==7, \%$ hyperbola points $\left(\mathrm{y}=\left(\mathrm{xmax}^{\wedge} 2+\mathrm{xmax} \wedge 2 / \mathrm{a}^{\wedge} 2 *\left(\mathrm{x}^{\wedge} 2\right)\right)-0.5-\mathrm{a}+\mathrm{d} / 2\right)$

$\left.f=\left(x \max \wedge 2+x \max \wedge 2 / a^{\wedge} 2 *(x x \cdot * x x)\right)\right)^{\wedge}(0.5)-x \max +d / 2 ;$

end 
$\%$ shapesfundersq.m

\% Crian Wessel
$\%$ of the futes the square root of the derivative

function $f=$ shapesfundersq ( $x x, n d p, a, x \max$ );

$\%$ sheet points

end

$\%$ circle points
if ndp $==4$

if $\underset{f}{\mathrm{xax}}=\mathrm{x}=\mathrm{xx}$

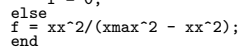

end

\%parabola pts
if ndp $=5$

$\begin{aligned} \operatorname{ndp} & ==5 \\ f & =a^{\wedge} 2 * 4 * x x^{\wedge} 2\end{aligned}$

end

$\%$ ellipse points
if ndp $==6$

$f=x x^{\wedge} 2 * x \max x^{\wedge} 2 / a^{\wedge} 2 *\left(a^{\wedge} 2-x x^{\wedge} 2\right)^{\wedge}-1 ;$

end

$\%$ hyperbola points
if ndp $==7$

$f=x x^{\wedge} 2 * x \max { }^{\wedge} 2 / a^{\wedge} 2 *\left(a^{\wedge} 2+x x^{\wedge} 2\right)^{\wedge}-1 ;$

end 


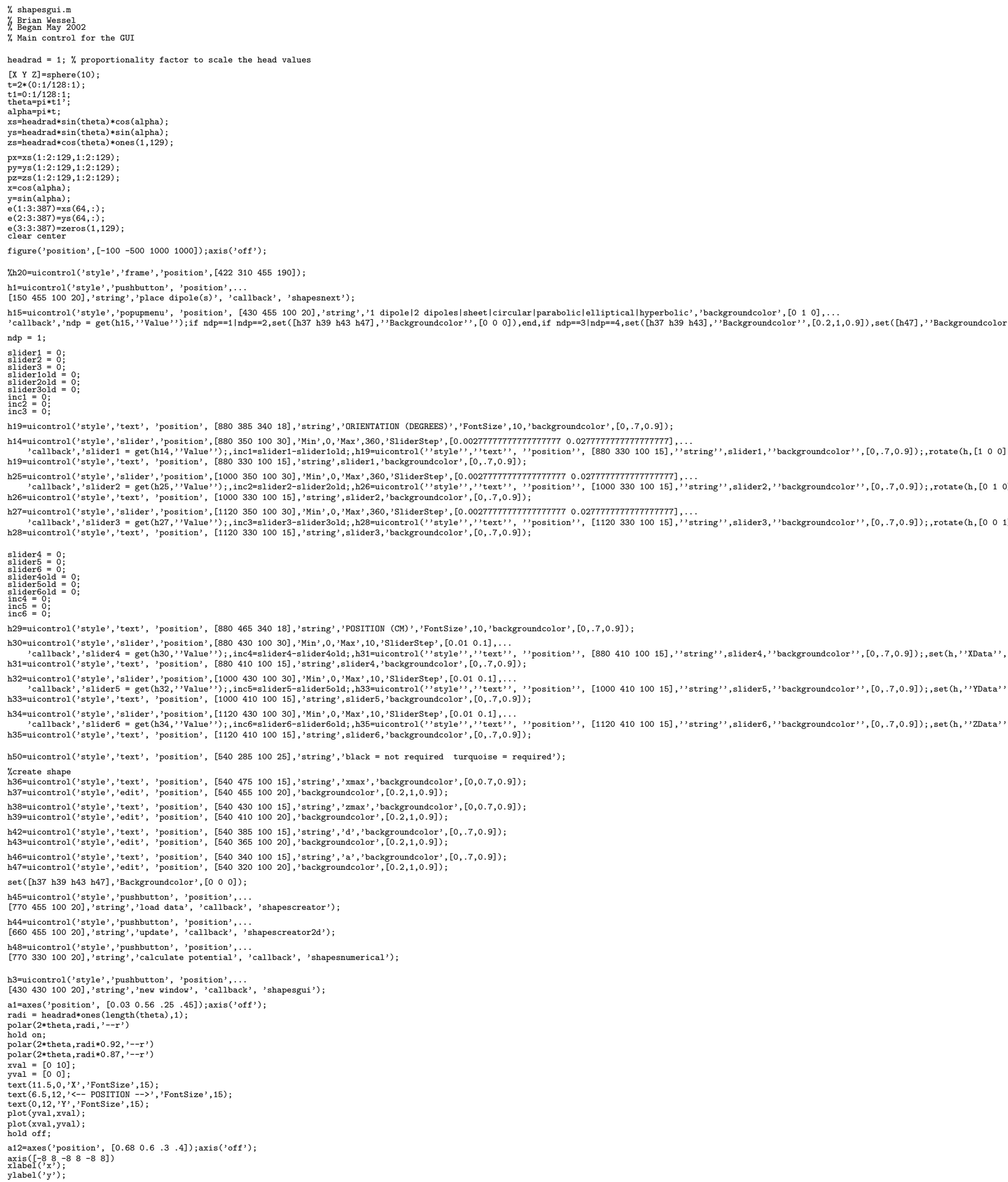


Zlabel ('z');
rotate3d;

a14=axes ('position', [0.35 0.6 .3.4]); axis ('off');

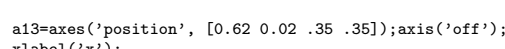
xlabel (' $x$ '):
ylabel ('y')

zlabel $z_{z}$

a18=axes('position', $\left[\begin{array}{llll}0.02 & 0.20 & .3 & 0.3\end{array}\right]$ ); $\operatorname{axis}($ 'off'), rotate3d 


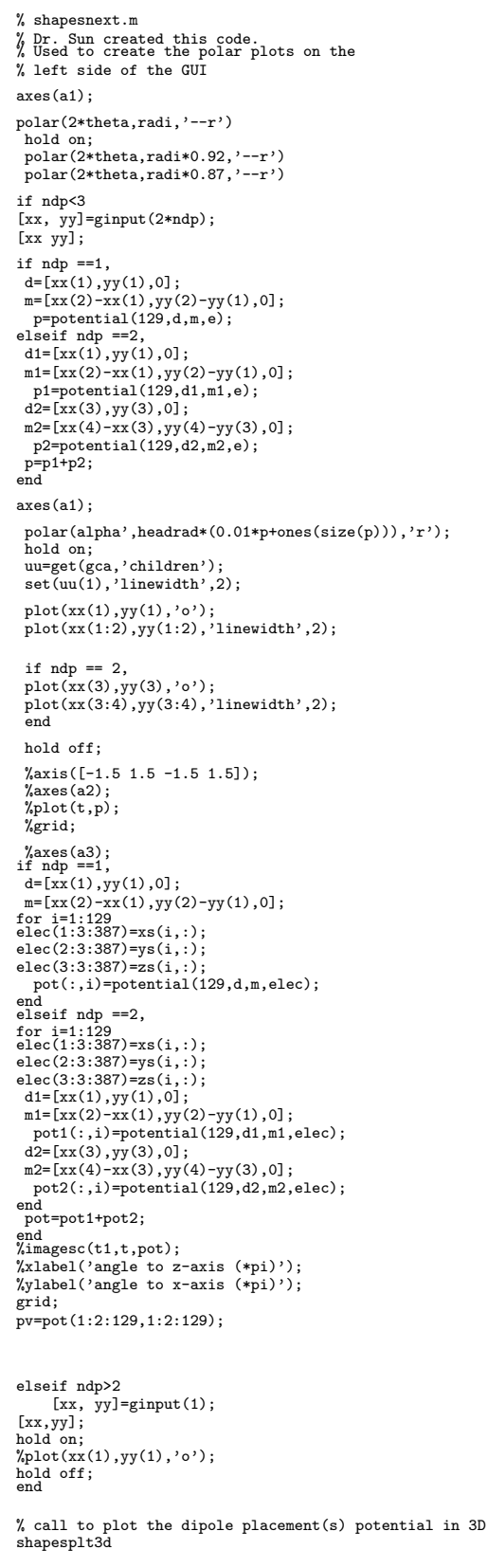




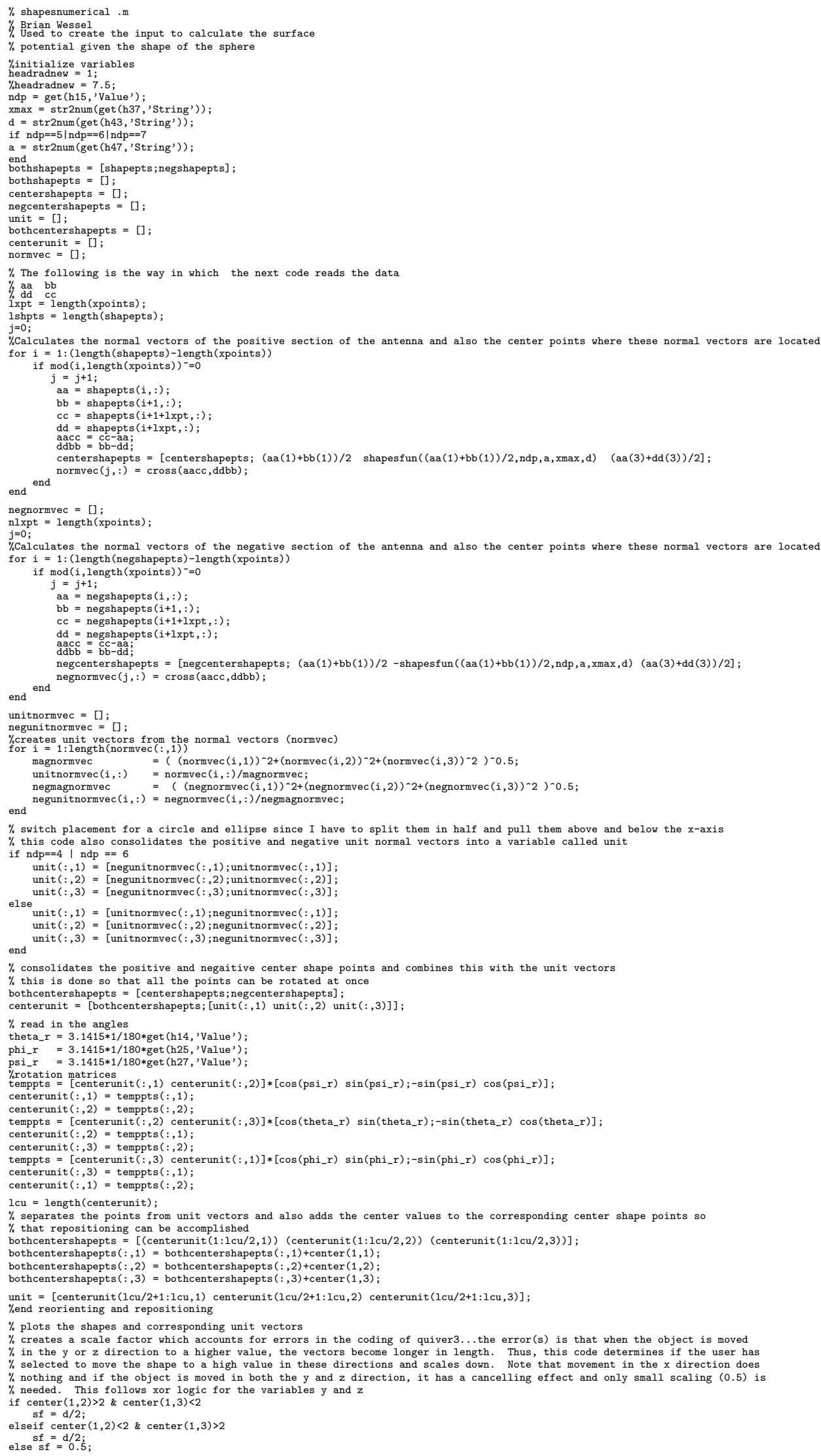


end

$\%$ don't need this now that I'm not using dipoles

\% axes (a14)

$\%$ axis equal

$\%$ sold on scatter (bothcentershapepts (: , 1), bothcentershapepts (: , 2), bothcentershapepts (: , 3), 15, '--r') ;

$\%$ xlabel('x');

$\%$ zlabel ('z');

$\% \mathrm{xmin}=\min ($ bothcentershapepts $(:, 1))$;

$\%$ max $=\max ($ bothcentershapepts $(:, 1))$

$\%$ ymax $=\max ($ bothcentershapepts $(, 2)) ;$

$\% \mathrm{zmin}=\min ($ bothcentershapepts $(:, 3))$;

$\% \operatorname{zmax}=\max ($ bothcentershapepts $(:, 3))$;

$\% \operatorname{axis}([(x \min -(x \max -x \min ) / 2)(x \max +(x \max -x \min ) / 2)(y \min -(y \max -y \min ) / 2)(y \max +(y \max -y \min ) / 2) \quad(z \min -(z \max -z \min ) / 2) \quad(z \max +(z \max -z \min ) / 2)])$

$\%$ hold off

$\% \%$ used this code to make a figure for a paper

$\%$ quiver3 (bothcentershapepts (: , 1), bothcentershapepts (: , 2), bothcentershapepts (: , 3), unit (: , 1), unit (: , 2), unit (: , 3), sf)

$\%$ axis equal

$\%$ hold on 3 scatter (bothcentershapepts (: , 1), bothcentershapepts (: , 2), bothcentershapepts (: , 3), 15, '--r') ;

$\%$ xlabel ('x $(\mathrm{cm})$ ');
$\%$ ylabel ('y $(\mathrm{cm})$ ');
$\%$ zlabel ('z $(\mathrm{cm})$ ');

$\%$ xminel $=2(\mathrm{~cm}))$;

$\%$ max $=\min ($ bothcentershapepts $(:, 1))$;

$\%$ ymin $=\min ($ bothcentershapepts $(:, 2)) ;$

$\%$ ymax $=\max ($ bothcentershapepts $(:, 2)) ;$

$\% \mathrm{zmax}=\max ($ bothcentershapepts $(:, 3))$.

$\% \% \operatorname{axis}([(x \min -(x \max -x \min ) / 2)(x \max +(x \max -x \min ) / 2) \quad(y \min -(y \max -y \min ) / 2)(y \max +(y \max -y \min ) / 2) \quad(z \min -(z \max -z \min ) / 2) \quad(z \max +(z \max -z \min ) / 2)])$

$\%$ hold off

\% code to calculate the normal vectors according to MatLab - doesn't do a very good job

$\%$ newxpts $=[]$

$\begin{array}{ll}\text { newypts } & =[] ; \\ \% & \text { newzpts }=[] ;\end{array}$

$\%$ for $i=0:$ (length (centershapepts)/(length $(x p t)-1)-1)$

$\%$ newxpts $=[$ newxpts, centershapepts $((i *($ length $(x p t)-1)+1):(i+1) *($ length $(x p t)-1), 1)]$

$\begin{aligned} \text { newypts } & =\text { [newypts, centershapepts }((i *(\text { length }(x p t)-1)+1):(i+1) *(\text { length }(x p t)-1), 2)] \\ \text { newzpts }=[\text { newzpts, } & \text { centershapepts }((i *(\text { length }(x p t)-1)+1):(i+1) *(\text { length }(x p t)-1), 3)]\end{aligned}$

$\%$ end

\% figure(5)

\% [Nx, Ny, Nz] $=$ surfnorm (newxpts', newypts', newzpts');

$\%$ surfnorm (newxpts', newypts', newzpts')

$\% \% \%$ UNCOMMENT THE NEXT 60 LINES OR SO TO USE POTENTIAL.C

$\% \% \%$ clear totpoten

$\% \% \%$ sets up parametric values to be read into potential.meglx

$\% \% \% \% t=2 *(0: 1 / 128:(1-1 / 128)) ; 128$ pts code

$\% \% \% \mathrm{t}=2 *(0: 1 / 128: 1)$;

$\% \% \% \%$ t1 $=0: 1 / 128: 0.99999 ; 128$ pts code

$\% \% \% \mathrm{t} 1=0: 1 / 128: 1$;

$\% \% \%$ theta=pi*t $1{ }^{\prime}$;

$\% \%$ alpha=pi*t;

\%

$\% \%$ ys=headradnew

$\% \% \%$ zs=headradnew*cos (theta) $*$ ones $(1,128) ; 128$ pts code

$\% \% \%$ zs=headradnew

$\% \% \%$ poten $=[] ;$

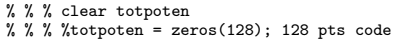

$\% \%$ totpoten $=$ zeros $(129)$

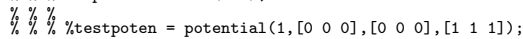

$\% \% \%$ for $j=1$ : length (unitnormvec)

$\% \% \%$ for $j=1: 1$

$\% \%$ for $\begin{aligned} & i=1: 129 \\ & \text { elec }(1: 3: 387)=x s(i,:)\end{aligned}$

$\% \% \% \quad$ elec $(2: 3: 387)=y s(i,:) ;$

$\% \% \quad \operatorname{poten}(:, i)=$ potential $(129$, bothcentershapepts $(j,:), u n i t(j,:), e l e c)$;

$\% \%$ end $\quad$ totpoten $=$ totpotentpoten;

$\% \% \%$ end

$\% \% \%$ create a dummy variable to be graphed so as to reinitialize axes a13

$\% \% \%$ testpoten $=$ zeros (129)

$\% \% \%$ axes (a13)

$\% \% \%$ px=xs $(1: 2: 129,1: 2: 129) ;$ py=ys $(1: 2: 129,1: 2: 129) ; p z=z s(1: 2: 129,1: 2: 129)$

$\% \% \%$ surface $(\mathrm{px}, \mathrm{py}, \mathrm{pz}, \mathrm{pv})$

$\% \% \%$ \% iaxes(a13)

$\% \% \% \mathrm{pv}=[] ;$

$\% \% \%$ \% imagesc (t1, t, totpoten)

$\% \% \%$ px=xs $(1: 2: 129,1: 2: 129) ; p y=y s(1: 2: 129,1: 2: 129) ; p z=z s(1: 2: 129,1: 2: 129)$;

$\% \%$ surface (px,py,pz,pv');

$\% \% \%$ rotate $3 d ;$

$\% \%$ shading interp

$\% \%$ colormap ('jet')

$\% \% \%$ \%colorbar (cm) ', 'FontSize', 15$)$;

$\% \% \%$ ylabel('y $(\mathrm{cm})$, ','FontSize,, 15$)$;

$\% \%$ set(gca,'Visible', 'On')

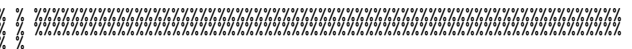

$\% \% \%$ Using Ernest Frank's arbitrary placed and spaced dipole (this code does not use the Mex function, thus it is slow)

$\% \%$ clear Vr ra rb theta phi Px Py Pz theta phi

$\% \% \%$ radius of the sphere 


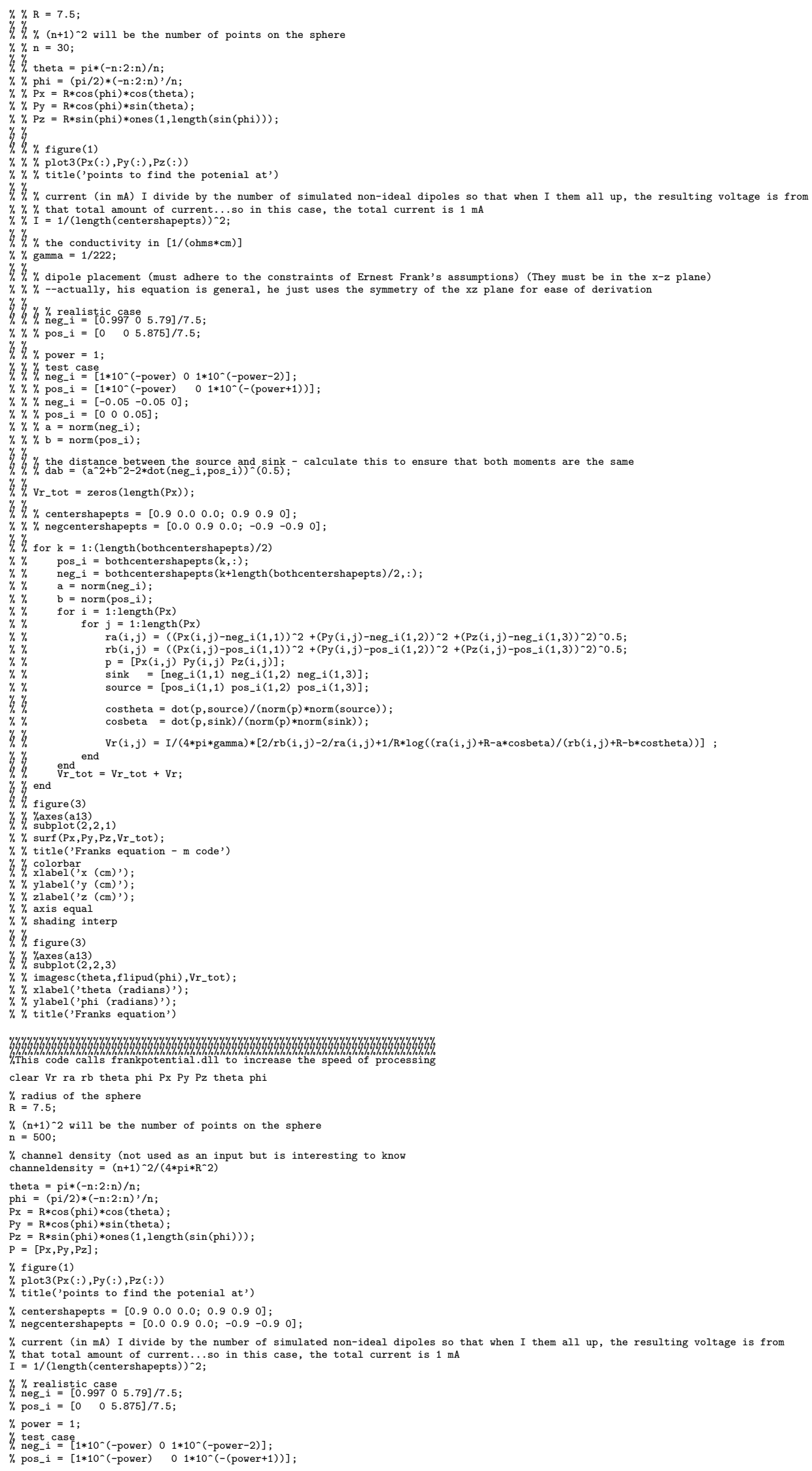


$\%$ neg_i $^{i}=\left[\begin{array}{lll}-0.05 & -0.05 & 0\end{array}\right] ;$
$\%$ pos_i

$\%$ a $=$ norm $\left(\right.$ neg_i $\left._{-1}\right)$

$\mathrm{k}=$ length (bothcentershapepts);

$\mathrm{Vr}=$ frankpotential $(\mathrm{I}$, bothcentershapepts $(\mathrm{k} / 2+1: \mathrm{k},:)$, bothcentershapepts $(1: \mathrm{k} / 2,:), \mathrm{P}, \mathrm{R})$;

$\%$ figure (3)

\%subplot $(2,2,2)$

$\%$

(ion - c code')

colorbar $\left.\left.{ }_{\text {xlabel('x }(\mathrm{cm})}\right)^{\prime}\right)$;

ylabel ('y $(\mathrm{cm})$ '):

axis equal $(\mathrm{cm})$ ')

shading interp

$\%$ figure (3)

$\%$ subplot $(2,2,4)$

$\%$ imagesc (theta,flipud (phi), $\mathrm{Vr}$ )

$\%$ xlabel ('theta (radians)')

$\%$ title('pranks equation')

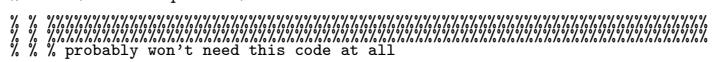

$\% \% \%$ new code to call inhomogeneous (4 shell) model

$\% \%$ clear poten

$\% \%$ clear totpoten

$\% \%$ MXPTS $=11$;

$=2 * 3.14 / \mathrm{MXPTS}$

$\% \%$ a = fix (MXPTS);

$\% \% x=[] ;$

$\% \% z=[]$

$\% \%$ for $t=0:($ MXPTS -1$)$
for $p=0:(a-1)$

$\begin{array}{ll}\% & \text { theta }=\text { increment } * t \\ \% & \text { phi }=\text { increment } * \text { p }\end{array}$

$\% \% \quad x=[x, \cos (p h i) * \sin ($ theta $)] ;$

$\begin{array}{ll}\% \% & y=[y, \sin (p h i)] ; \\ \% \% & z=[z, \cos (p h i) * \cos (\text { theta })]\end{array}$

$\% \%$ end end

$\% \%$ figure $(99)$

\% scatter $3(\mathrm{x}, \mathrm{y}, \mathrm{z})$;

$\% \% \% x=\left[\begin{array}{llllll}0 & 0 & 0 & 0 & 0 & 0\end{array}\right] ;$

$\% \% \% \mathrm{z}=\left[\begin{array}{llllll}2 & 2 & 2 & 2 & 2 & 2\end{array}\right] ;$

$\% \%$ totpoten $=\operatorname{zeros}(6,1)$;

$\% \%$ for $j=1:$ length(bothcentershapepts)

$\% \% \%$ for $j=1: 6$

$\% \%$ poten $=$ pot_vec_order $3\left(121, \operatorname{bothcentershapepts}(j,:)\right.$, unit $\left.(j,:),\left[x^{\prime} y^{\prime} z^{\prime}\right]\right)$;

$\%$ totpoten = totpoten + poten; 
$\%$ shapesplt3d.m

\% Usian wessel

$\%$ below the polar plot

axes (a18)
rotate3d

surface (px,py,pz,pv')

shading interp

xlabel(' $x$ ');

ylabel('y');

zlabel(' $z$ ')

set (gca,'Visible', 'on') 


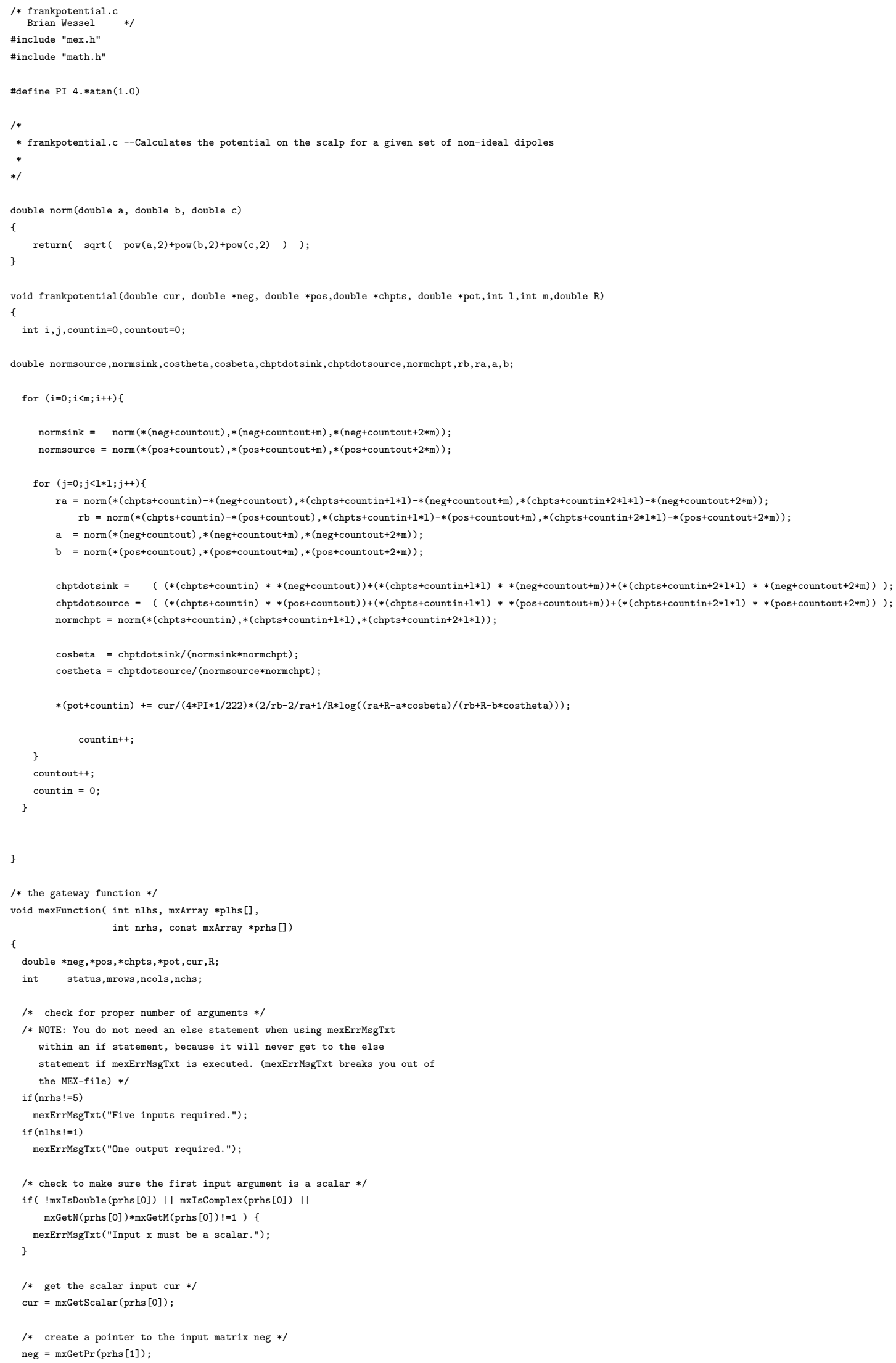




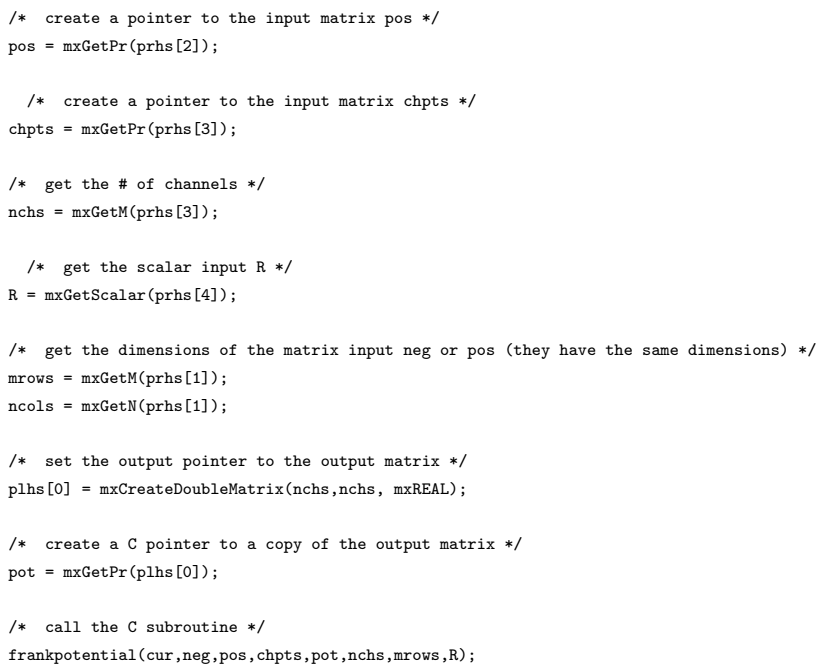




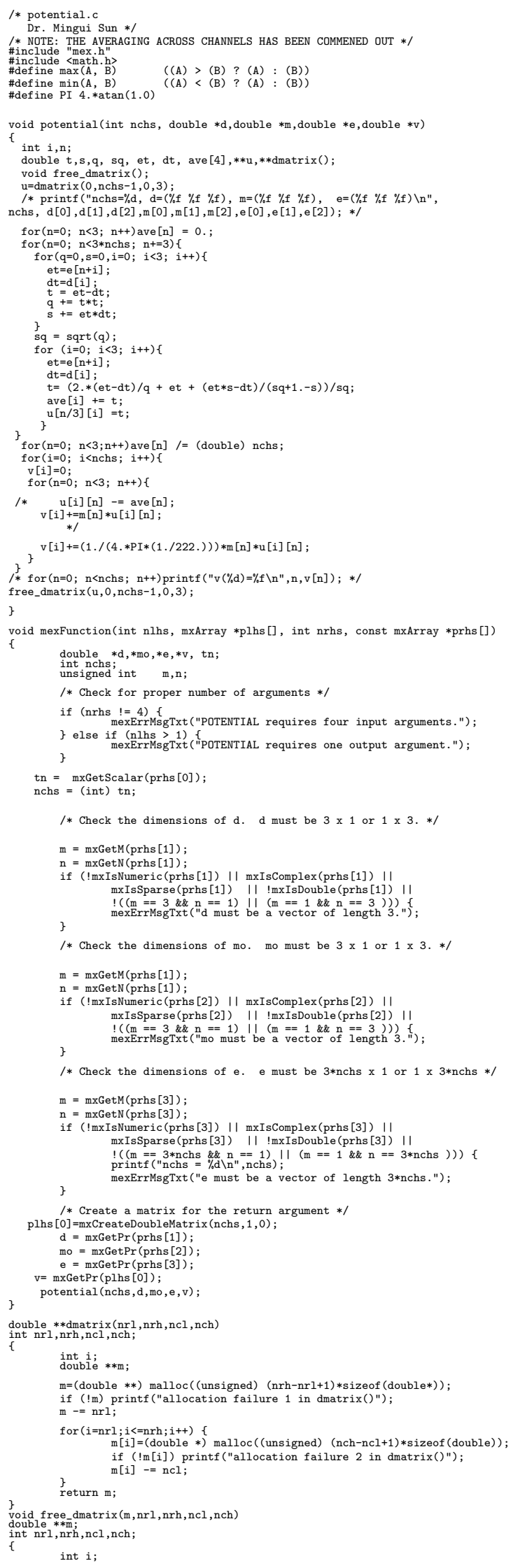


for (i=nrh;i>=nrl;i--) free((char*) (m[i]+ncl)); 


\section{.2 SHAPE OPTIMIZATION}

Contains the following files:

- shapesfundersq.m (refer to .1)

- shapesoptimize_sym.m

- shapesptsopt_v2.m

- shapestotarclength.m

- frankpotential.c (refer to .1) 


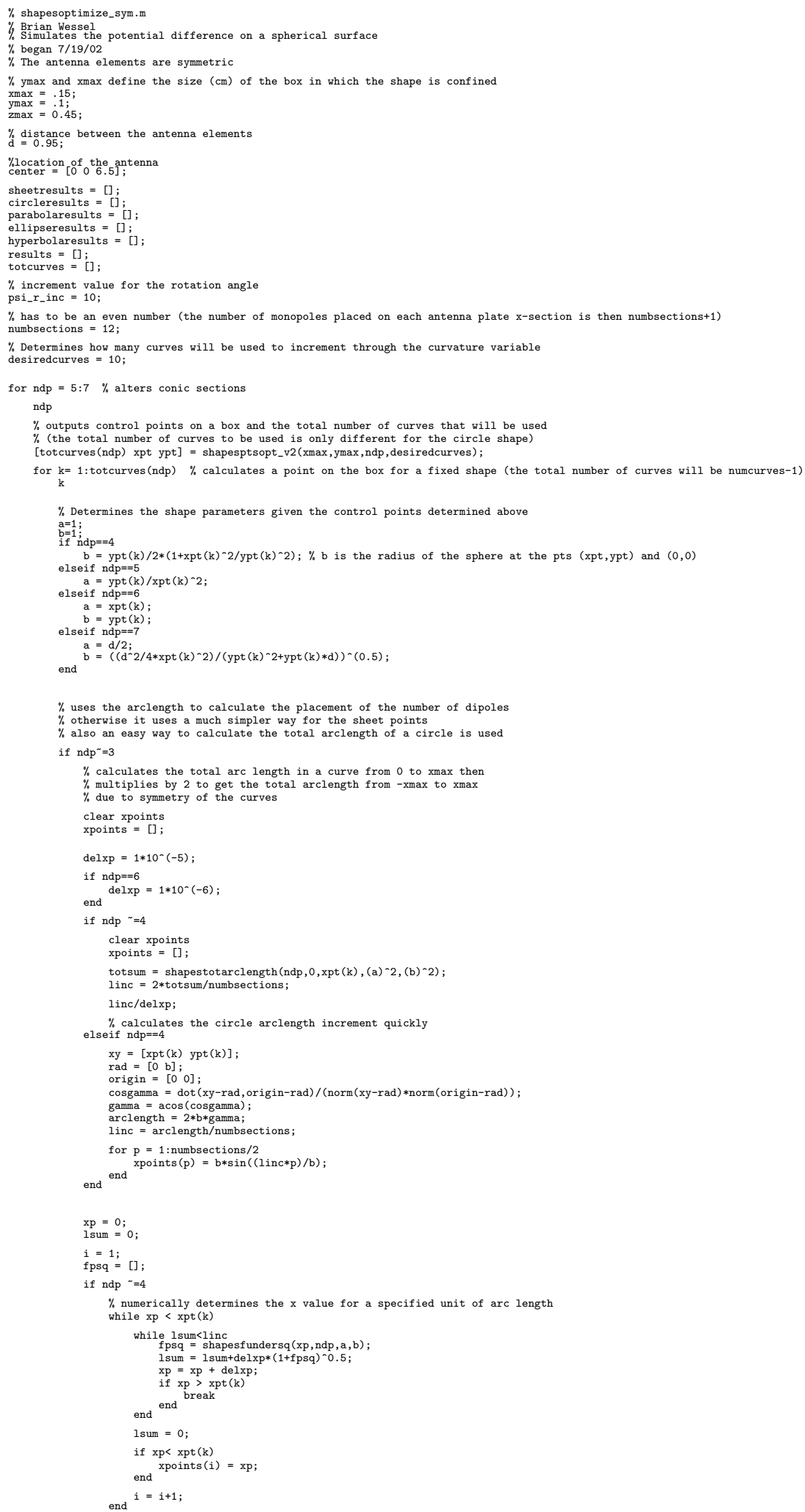




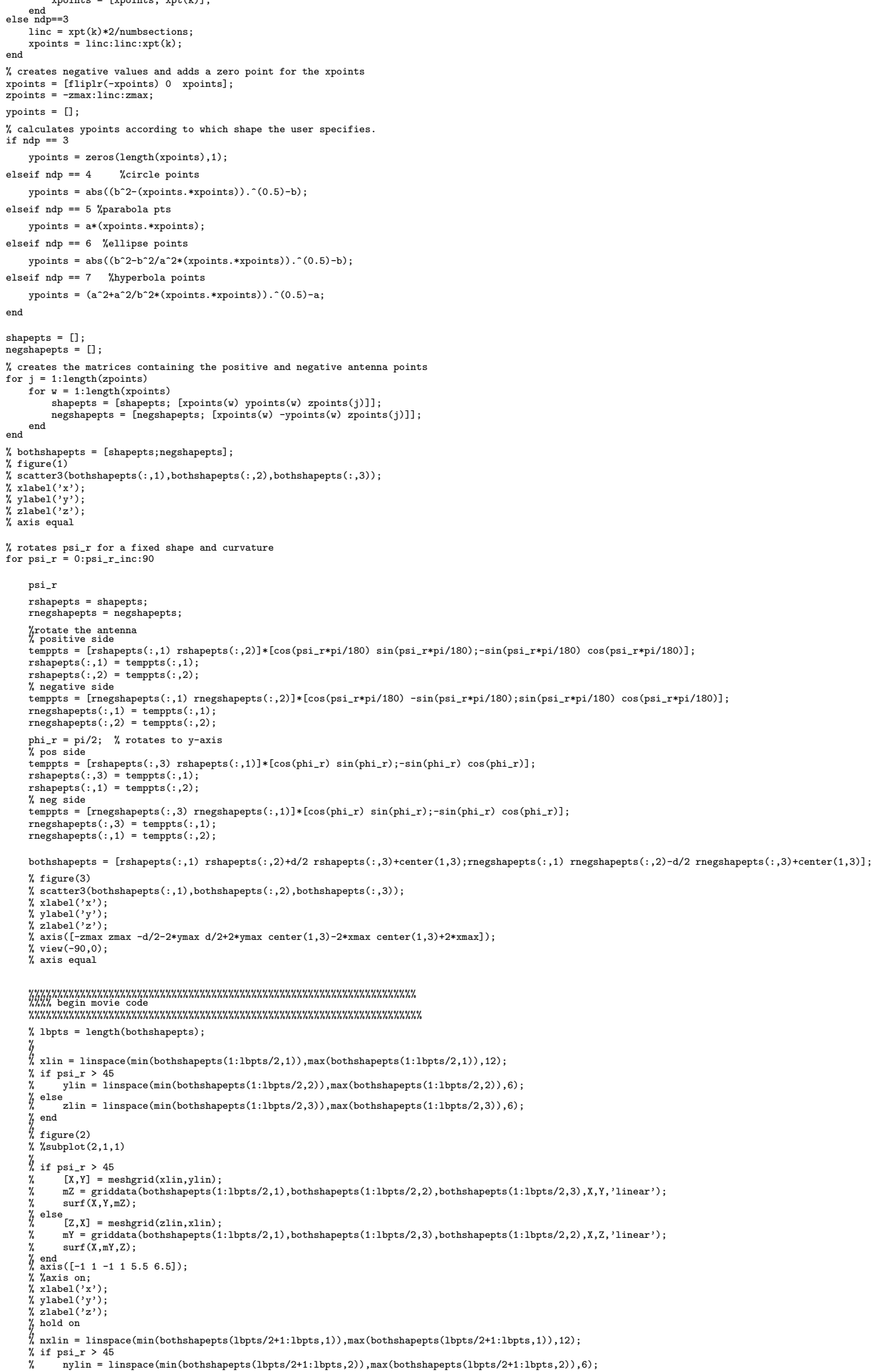




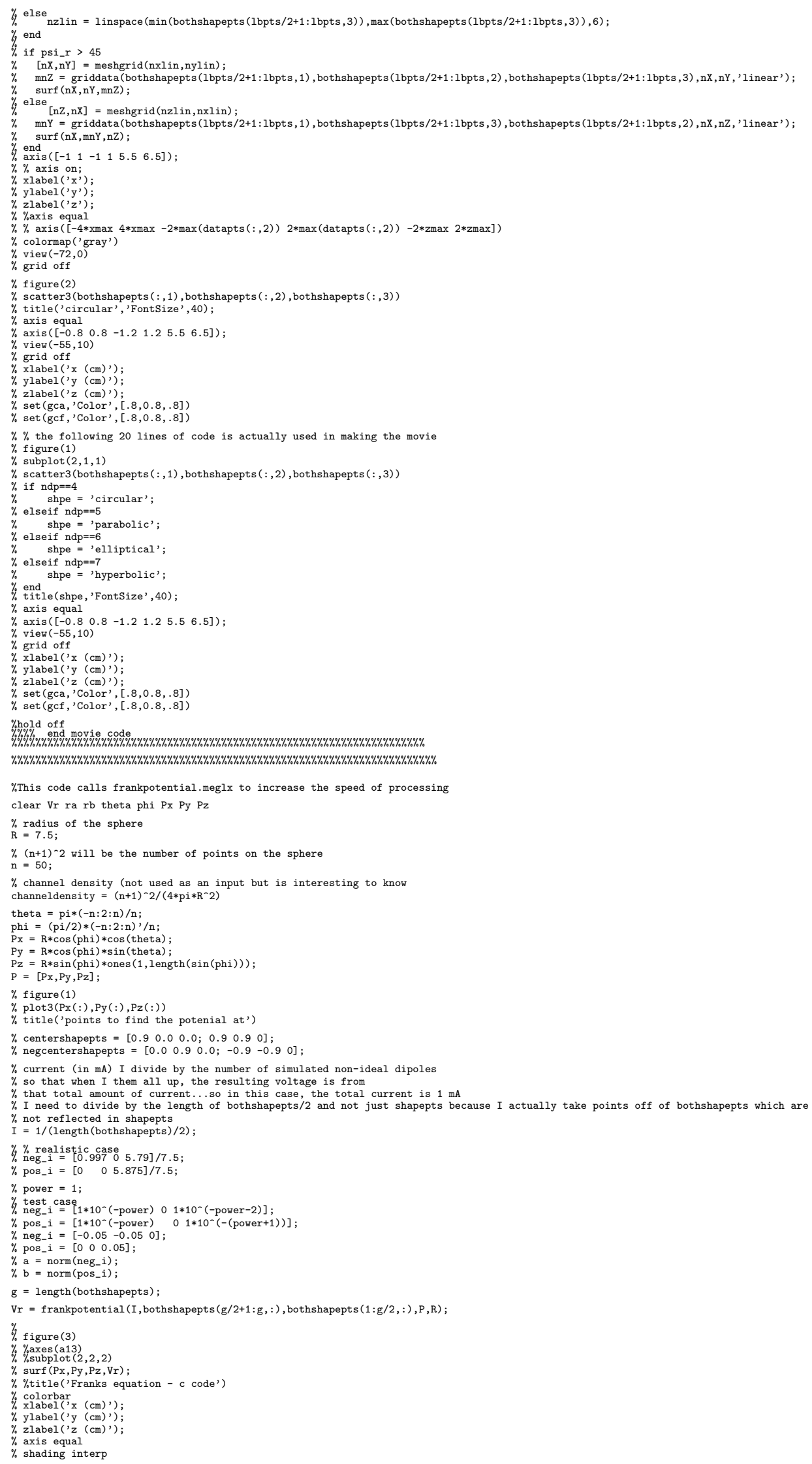




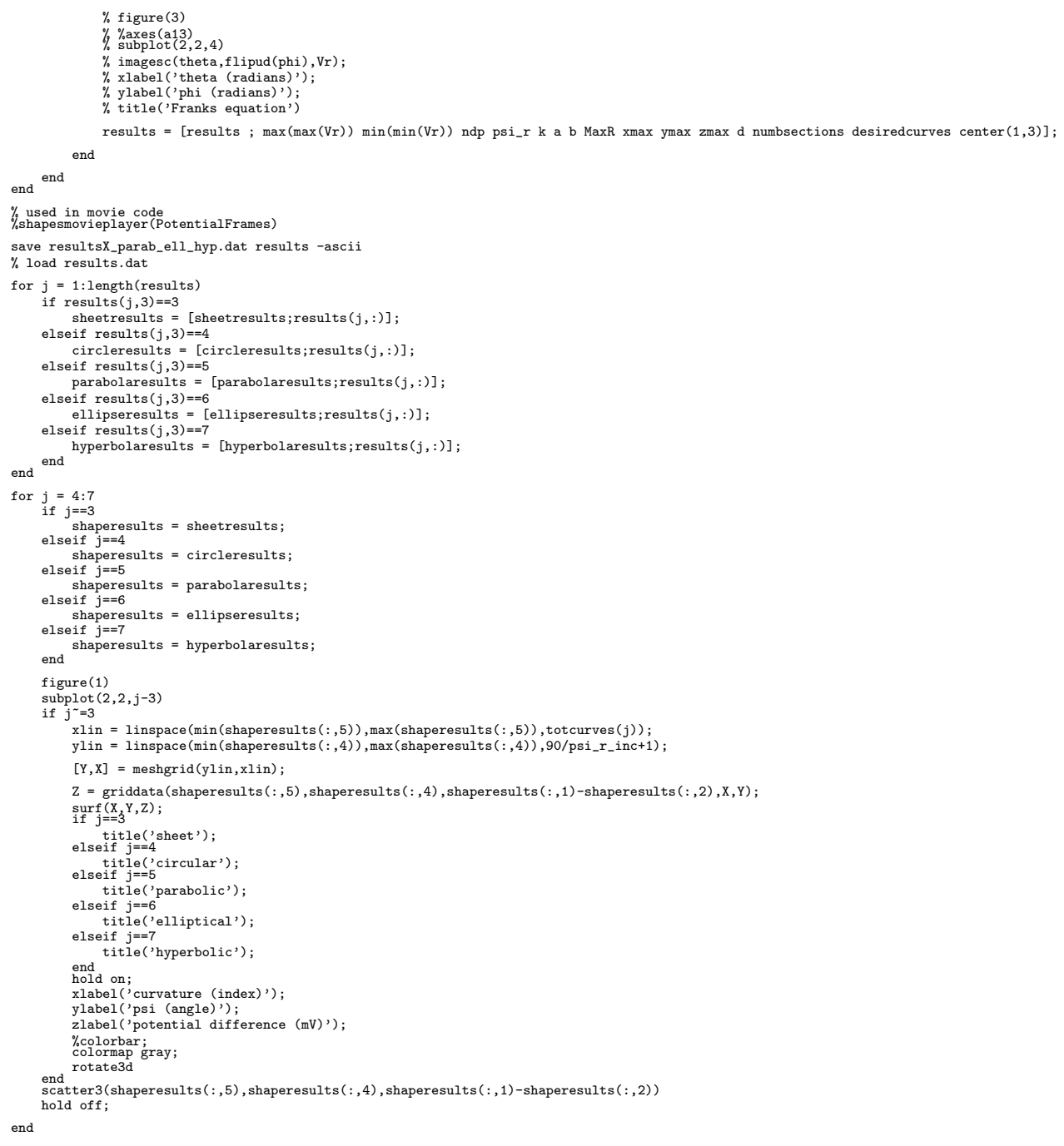$$
\begin{aligned}
& \text { \%colorbar; } \\
& \text { colormap gray }
\end{aligned}
$$$$
\text { rotate3d }
$$

end
scatter3 (shaperesults $(:, 5)$, shaperesults $(:, 4)$, shaperesults $(:, 1)$-shaperesults $(:, 2)$ )
hold off;

end 


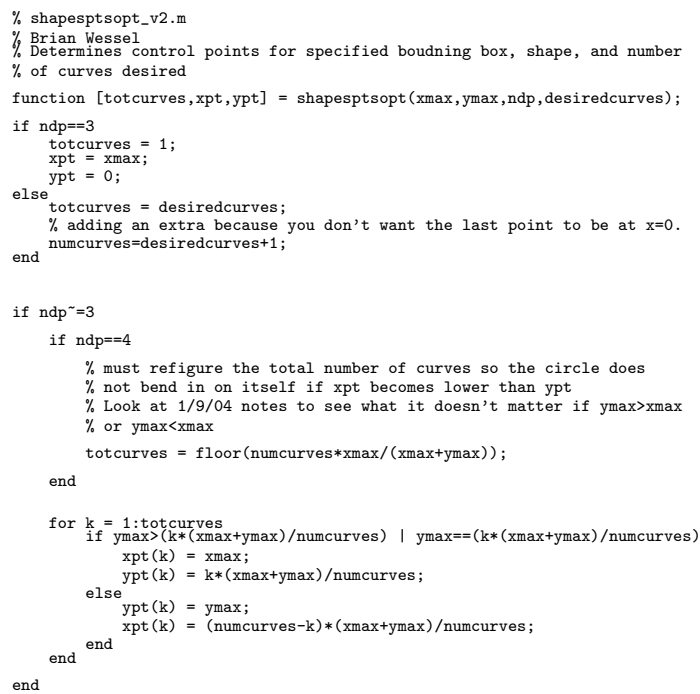

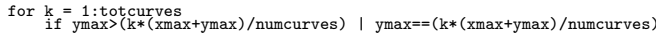
$\operatorname{xpt}(k)=x \max$

$\mathrm{t}(\mathrm{k})=\mathrm{k} *(\mathrm{xmax}+\mathrm{ymax}) /$ numcurves;

$\begin{aligned} \operatorname{ypt}(\mathrm{k}) & =\mathrm{ymax} ; \\ \mathrm{xpt}(\mathrm{k}) & =(\text { numcurves }-\mathrm{k}) *(\mathrm{xmax}+\mathrm{ymax}) / \text { numcurves; }\end{aligned}$

end

end 


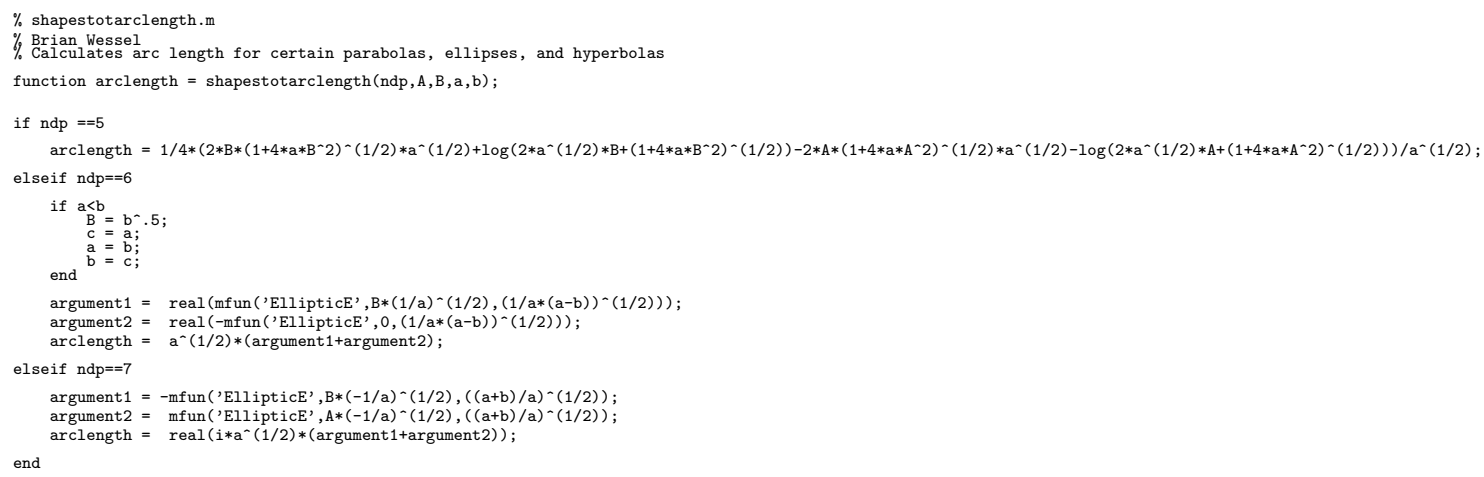




\section{.3 FE SHAPES CREATION}

Contains the following files:

- create_antennas_ptcontrol_v5.m

- normalpointsv2.m

- results_shapes.m

- shapesfundersq.m (refer to .1)

- shapesptsopt_v2.m (refer to .2)

- shapestotarclength.m (refer to .2)

- view_solution_Max.m 


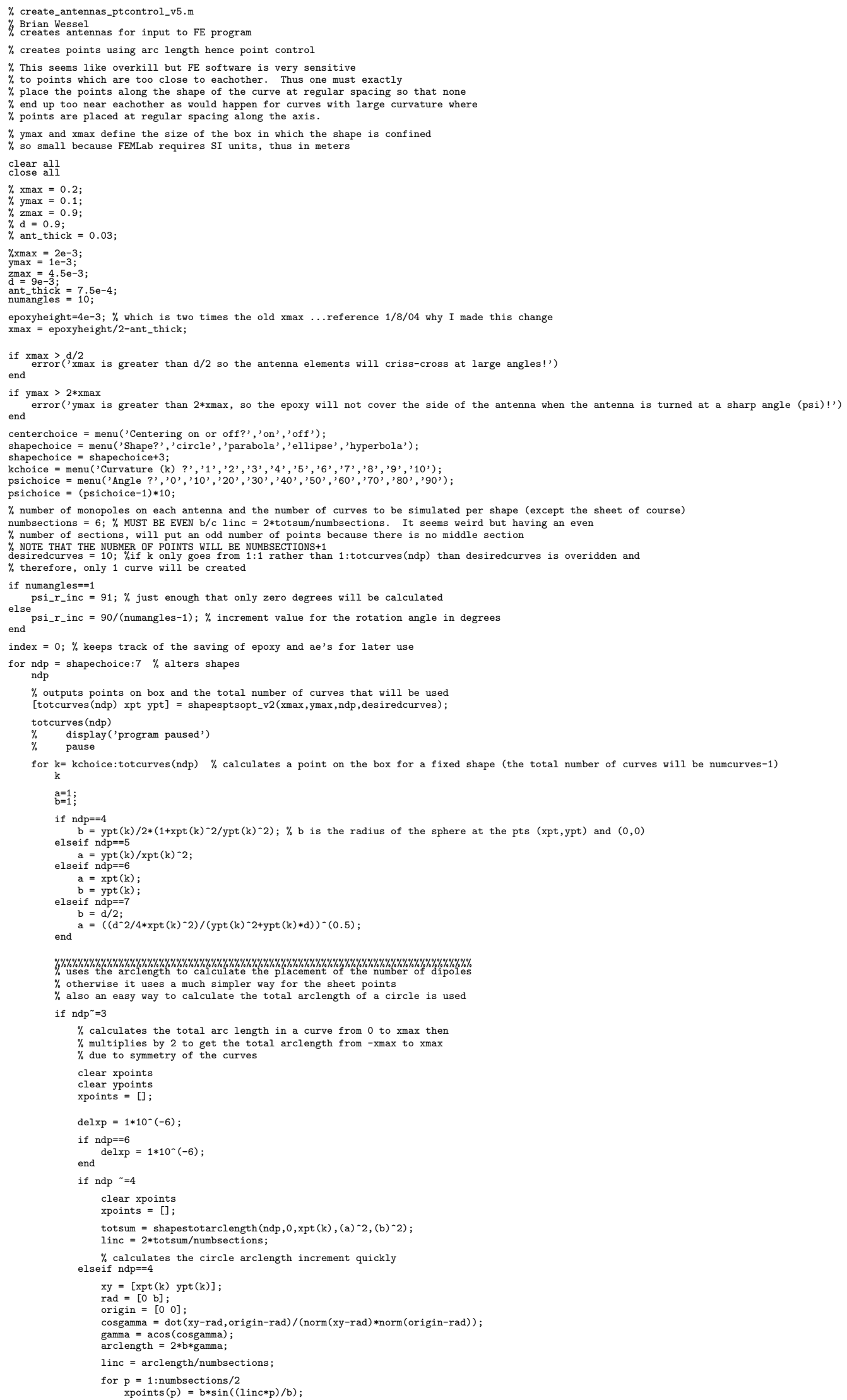




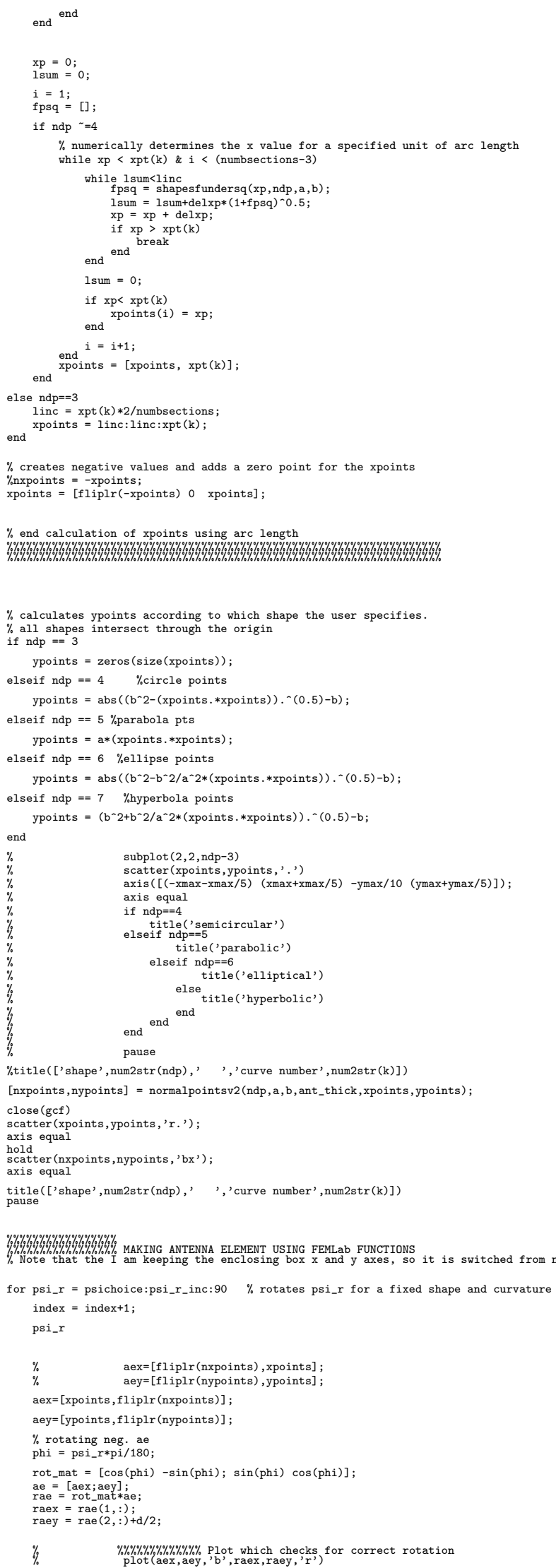




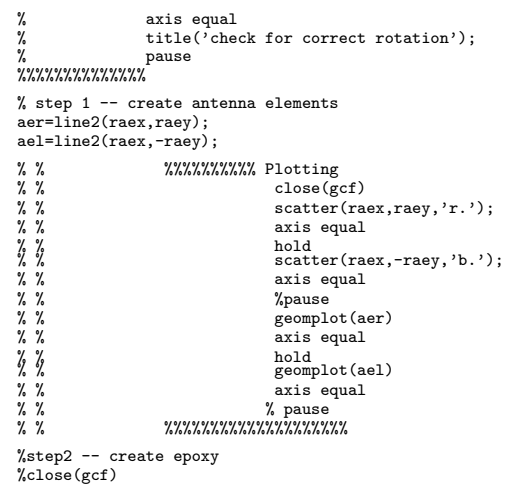

$\% \% \% \% \% \% \% \% \% \% \% \% \% \% \% \% \% \% \% \% \%$ code for creating outside antenna first . . uses output of normalptsv2.m $\% \% \% \% \% \% \% \% \% \% \% \% \% \% \% \% \% \% \% \% \% \%$ half $=$ raex (numbsections $+2:$ length $($ raex $)) ;$
halfy $=$ raey(numbsections $+2:$ length $($ raey));

halfy $=$ raey (numbsections +2 : length(raey));
if centerchoice $=1 \%$ This choice will center the antenna element within the epoxy

\% This regime is fundamentally different from noncentering because it should expose

$\%$ the difference between having a shietd of epoxy us free space and will give insight

$\%$ if psi_r $=0$, we don't want to add extra epoxy or it will cause an error because the points

$\%$ will be too close to connect with a line... however, I should add about half the antenna thickness

$\%$ elliptically shaped antenna elements

if $\mathrm{psi}_{-} \mathrm{r}==0$

xtra $=$ ant_thick $/ 2$

xtra $=$ ant_thick $* 4 / 5$

totx $=[$ halfx (halfx(length(halfx))-xtra) (halfx (length(halfx))-xtra) fliplr(halfx) (halfx(1)+xtra) (halfx(1)+xtra) ];

toty $=$ [halfy halfy (length
epoxy = line2(totx, toty);

else $\%$ (for old way \#1)this strange sin*blah is so that at large angles the epoxy does not overlap itself and

$\%$ therefore cause a problem

$\%$ old way $\# 1--$ xtra $=$ halfx $(1,1)+\sin ($ psi_r*pi/180 $) *($ ymaxtant_thick $)$

$\%$ xtra $=\min ($ raex $)+2 * x \max +$ ant_thick

half $=$ fliplr(halfx)

halfy $=$ fliplr(halfy);
widthant $=\max ($ halfx $)$-halfx (1)

widthant $=\max ($ half $\mathrm{x})$-halfx $(1)$
xout $=$ ant

xout $=$ ant_thick $* \cos ($ phi $) * 3 / 5$;

$\begin{aligned} \mathrm{xtra} & =(\text { epoxyheight-widthant }) / 2 \\ \mathrm{xtra} & =(\text { epoxyheight-widthant }) * 4 / 5\end{aligned}$

xtra $=$ (epoxyheight-widthant $) * 4 / 5$

yout $=$ ant_thick $* \sin (\mathrm{phi}) ;$
yout $=$ ant_thick $*$ sin $($ phi $) * 3 / 5 ;$

totx $=[$ half $\max ($ half $x)(\max ($ half $x)+x t r a)(\max ($ halfx $)+x t r a) \max ($ halfx $)$ fliplr(halfx) halfx(1) (halfx(1)-xtra) (halfx(1)-xtra) halfx(1)]

$\% \%$ Sophisticated Modeling which is memory intensive

totx $=$ half $x$
toty $=$ halfy

totx $=[\operatorname{totx}(\max ($ hall $\mathrm{x})+\mathrm{xout})]$

totx $=[$ totx (halfx (length(half $x))+$ xout) $)$
toty $=[$ toty (halfy(length(halfy))+yout) $]$

totx $=[$ totx (max (halfx) + xtra) $)]$
toty $=[$ toty (halfy $($ length (halfy) $)+$ yout $)]$

totx $=[\operatorname{tot} x(\max ($ half $\mathrm{x})+\mathrm{xtra})] ;$

(thalfy))+yout)]

tot $x=[\operatorname{tot} x(\max ($ half $x)+x$ out $)] ;$

$\begin{aligned} \text { totx } & =[\text { totx }(\text { half } x(\text { length }(\text { half } x))+\text { xout })] \\ \text { toty } & =[\text { toty }-(\text { halfy }(\text { length }(\text { halfy }))+\text { yout })]\end{aligned}$

$\begin{aligned} \text { totx } & =[\text { totx fliplr(half } x)] ; \\ \text { toty } & =[\text { toty -fliplr(halfy) }] ;\end{aligned}$

totx $=[$ totx $($ halfx $(1)-$ xout $)] ;$
toty $=[$ toty $-($ halfy $(1)-$ yout $)] ;$

totx $=[\operatorname{totx}($ halfx $(1)-x \operatorname{tra})] ;$

toty $=[$ toty $-($ halfy $(1)-$ yout $)] ;$

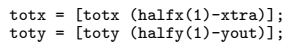

totx $=\left[\begin{array}{ll}\text { totx } & (\text { halfx }(1)-\text { xout })\end{array}\right] ;$
toty $=[\operatorname{toty}($ halfy $(1)-$ yout $)] ;$

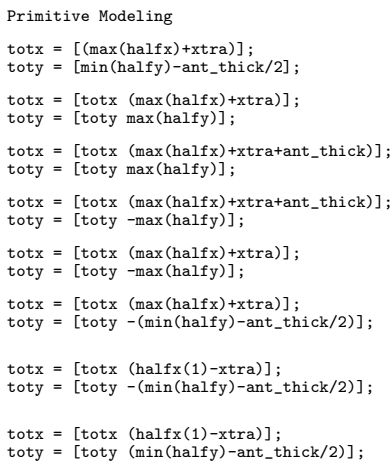




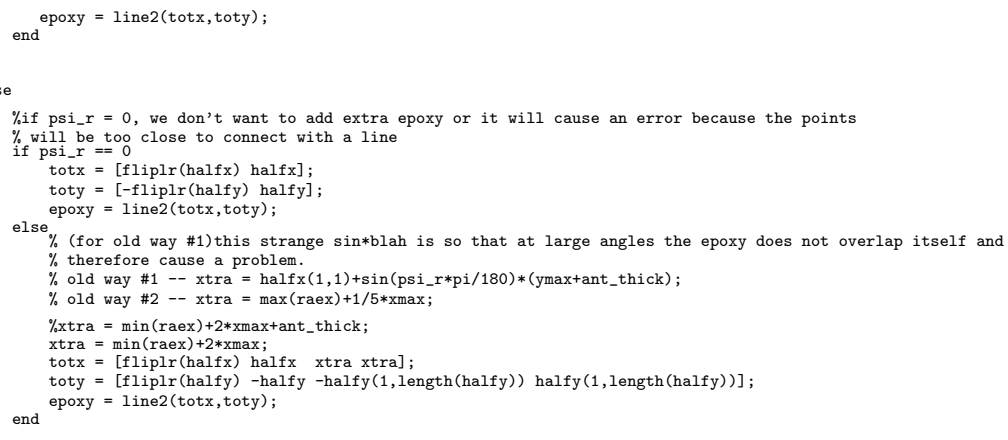

end $\% \% \% \% \% \% \% \% \% \% \% \% \% \% \% \% \% \% \% \% \%$ end create outside antenna first $\% \% \% \% \% \% \% \% \% \% \% \% \% \% \% \% \% \% \% \% \% \% \% \%$

$\% \% \% \% \% \% \% \% \% \% \% \% \% \% \% \% \% \% \% \% \% \% \% \% \% \% \% \% \%$ code for creating inside antenna first . . uses output of normalptsv4 m $\% \% \% \% \% \% \% \% \% \% \% \% \% \% \% \% \% \% \% \% \% \% \% \% \% \% \% \% \% \%$

$\% \% \quad$ halfx $=$ raex $(1:$ numbsections +1$) ;$

$\begin{array}{ll}\% \% & \text { half } x=\text { raex }(1: \text { numbsections }+1) ; \\ \% & \text { halfy }=\text { raey }(1: \text { numbsections }+1) ;\end{array}$

$\% \% \quad$ if centerchoice=1\% This choice will center the antenna element within the epoxy

$\%$ This regime is fundamentally different from noncentering because it should expose

$\%$ the difference between having a shield of epoxy vs free space and will give insight

$\%$ if psi_ $r=0$, we don't want to add extra epoxy or it will cause an error because the points

$\%$ will be too close to connect with a line

totx $=[f l i p l r($ half $x)$ half $x]$

toty $=[-f l i p l r(h a l f y)$ halfy $]$

epoxy $=1$ ine2 $($ tot $x$, toty $)$

$\%$ (for old way \#1)this strange sin*blah is so that at large angles the epoxy does not overlap itself and $\%$ therefore cause a problem.

$\%$ old way $\# 1--$ xtra $=$ half $x(1,1)+\sin ($ psi_r*pi/180 $) *(y \max +$ ant_thick $)$;

$\%$ xtra $=\min ($ raex $)+2 * x \max +$ ant_thick;

widthant $=\max ($ half $\mathrm{x})$-half $\mathrm{x}(1)$;

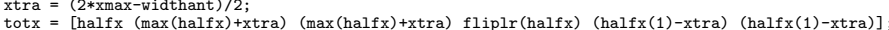

toty $=[$ halfy halfy(length(halfy)) -halfy(length(halfy)) -fliplr(halfy) -halfy(1) halfy(1)];

end

ety $=$ Thalfy halfy (length
epoxye2(tot, toty)

else

\%if psi_ $r=0$, we don't want to add extra epoxy or it will cause an error because the point

$\%$ will be too close to connect with a line

totx $=[f$ liplr(halfx) half $x ;$

toty $=[-f l i p l r(h a l f y)$ half $y]$

epoxy = line2(totx, toty);

else $_{\%}$ (for old way \#1) this strange sin*blah is so that at large angles the epoxy does not overlap itself and $\%$ therefore cause a problem.

for $(1,1)+\sin ($ psi_r $r *$ pi/180)* (ymax+ant_thick)

old way \#2 -- xtra $=\max (r a e x)+1 / 5 * x \max$;

$\%$ xtra $=\min ($ raex $)+2 * x \max +$ ant_thick;

xtra $=\min ($ raex $)+2 * x \max ;$

至

end

$\%$ \%\%\%\%\%\%\%\%\%\%\%\%\%\%\%\%\%\%\%\%\%\%\%\%\%\%\% end create inside antenna first $\% \% \% \% \% \% \% \% \% \% \% \% \% \% \% \% \% \% \% \% \% \% \% \% \% \% \%$

$\begin{array}{lc}\% & \% \% \% \% \% \% \% \% \% \% \% \% \% \% \% \text { Plotting } \\ \% & \text { geomplot (epoxy) } \\ \% & \text { title ( epoxy ') } \\ \% & \text { axis equal } \\ \% & \text { pause } \\ \% & \% \% \% \% \% \% \% \% \% \% \% \% \% \% \% \% \% \% \% \% \% \% \% \% \% \%\end{array}$

$\% \quad \% \% \% \% \% \% \% \% \% \% \% \% \% \% \% \% \% \% \% \% \% \% \% \% \% \% \% \% \% \% \% \% \% \% \% \% \% \% \% \% \% \% \% \% \% \% \% \% \%$ The following code should work but the epoxy cannot be extruded for some reason

$\begin{array}{ll}\% \% & \text { half }=\text { raex }(1 \text { : numbsections }+1) ; \\ \% \% & \text { width }=\text { half } 1,1) \text {-halfx }(1, \text { numbsections }+1) ;\end{array}$

if width $\begin{aligned} \text { totx } & =(2 * x \max \text {-xmax } / 10) \\ \text { thalf } & \text { fliplr(half } \mathrm{x})\end{aligned} ; \%$ the epoxy should be directly behind the ae's

toty $=[$ halfy -fliplr(halfy) $;$

else ${ }_{\text {extend }}=x \max -$ width $/ 2$;

leftx $=($-extend + half $x$ (numbsections +1$))$

en

[halfx(1) halfx fliplr(halfx) halfx(1) rightx rightx]

\%\%\%\%\%\%\%\%\%\%\%\%\%\%\%\%\%\%\%\%\%\%\%\%\%\%\%\%\%\%\%\%\%\%\%\%\%\%\%\%\%\%\%\%\%\%\%

$\%$ creating extrusion plane

C_wrkp=geomgetwrkpln ('quick', $\{$ 'xy', -zmax $\}$ )

$\%$ extruding epoxy and ant. elements

e_epoxy = extrude (epoxy, 'wrkpln', c_wrkp, 'distance', zmax*2);

e_aer = extrude(aer, 'wrkpln', ,c_wrkp, 'distance',, max $* 2)$
e_ael = extrude (ael, 'wrkpln', c_wrkp, 'distance', zmax $* 2$ );

$\%$ storing as positive and negative antenna elements and as insulation

pae $\left\{\right.$ index\} $=e_{-}$aer;

nae $\{$ index $\}=\mathrm{e}_{-}$ael;

$\% \% \% \% \% \% \% \% \% \% \% \% \% \% \% \% \%$ Plotting

$\% \% \quad$ close (gcf) 


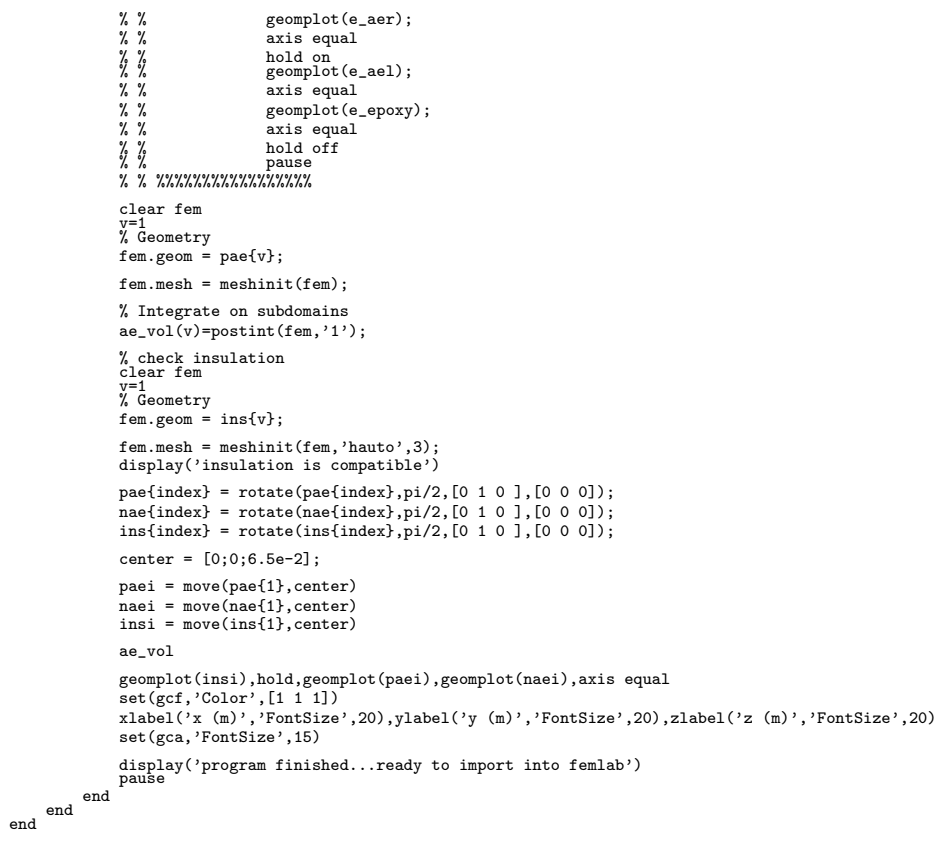


ylabel ('y (m)')
zlabel('z (m)')

hold

subindex $=$ subindex $x+1$

\% for $i=1$ : (numangles*totcurves (ndp)), geomplot (pae $\{i\})$, axis equal, pause, end 


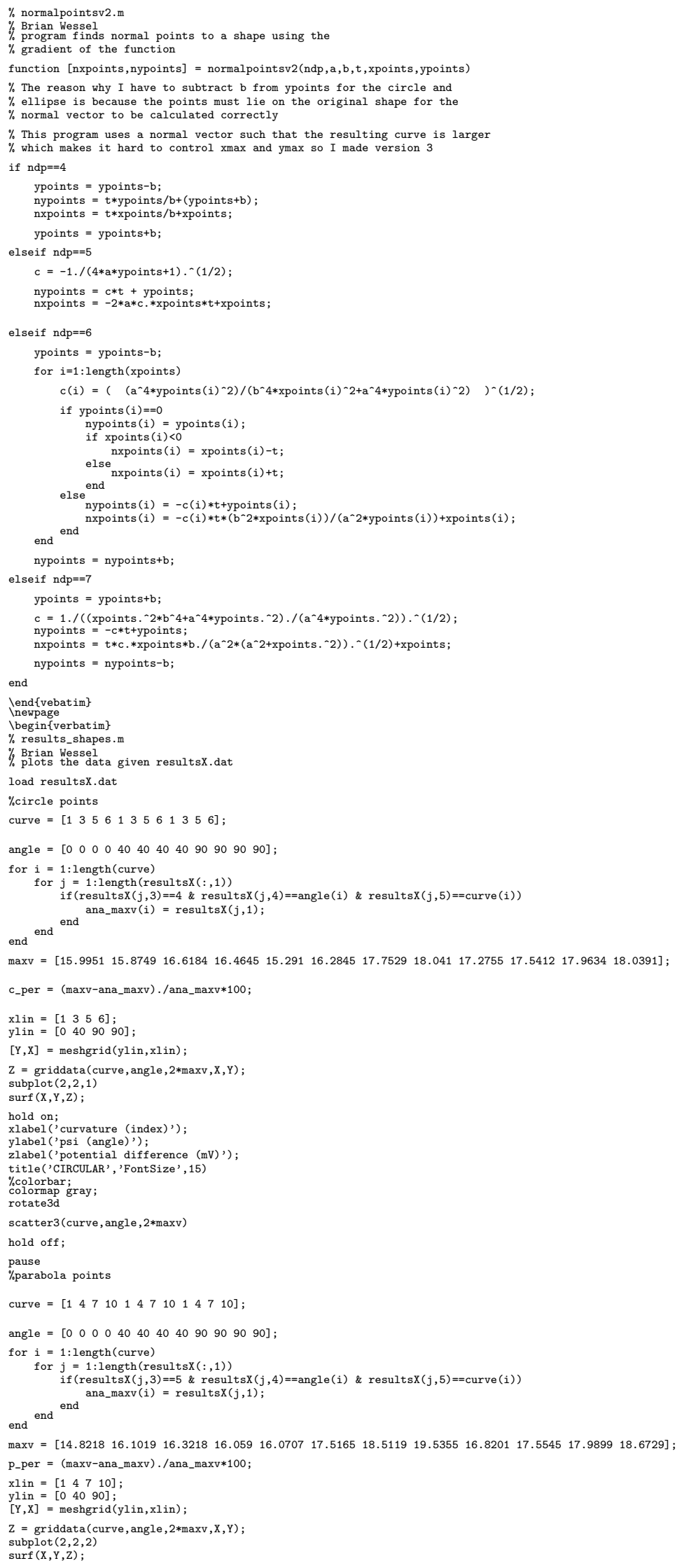




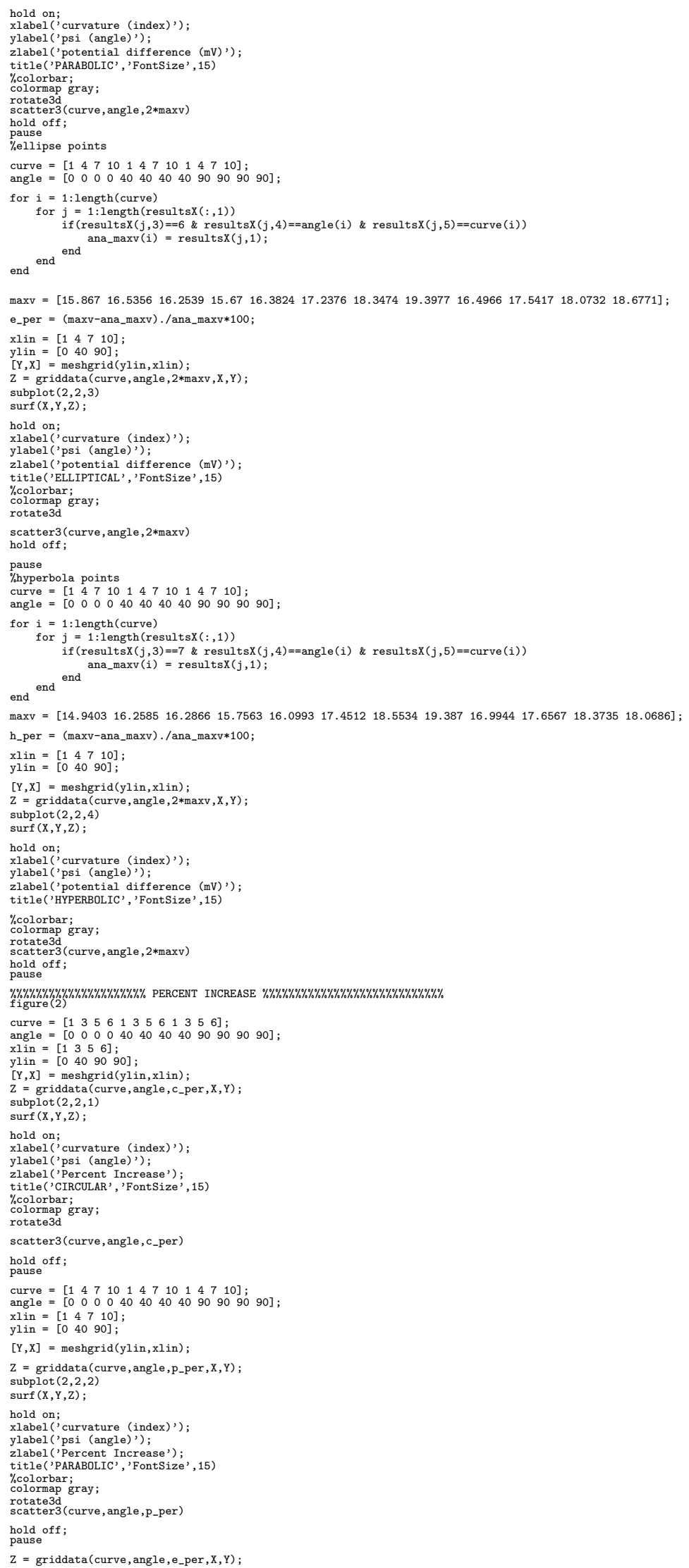


subplot $(2,2,3)$
$\operatorname{surf}(\mathrm{X}, \mathrm{Y}, \mathrm{Z})$

hold on

xlabel ('curvature (index)')

ylabel('psi (angle)');

zlabel ('Percent Increase');

\%colorbar;

rotate3d (curve, angle, e_per)

hold off

pause

subplot $(2,2,4)$

$\mathrm{Z}=$ griddata (curve, angle, $\mathrm{h}$ _per $, \mathrm{X}, \mathrm{Y}$ ) surf $(X, Y, Z)$

xlabel ('curvature (index)')
ylabel ('psi (angle)');

zlabel ('Percent Increase');
title('HYPERBOLIC','FontSize', 15 )

title ('HYPERBOLI

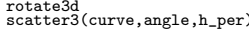

hold off; 


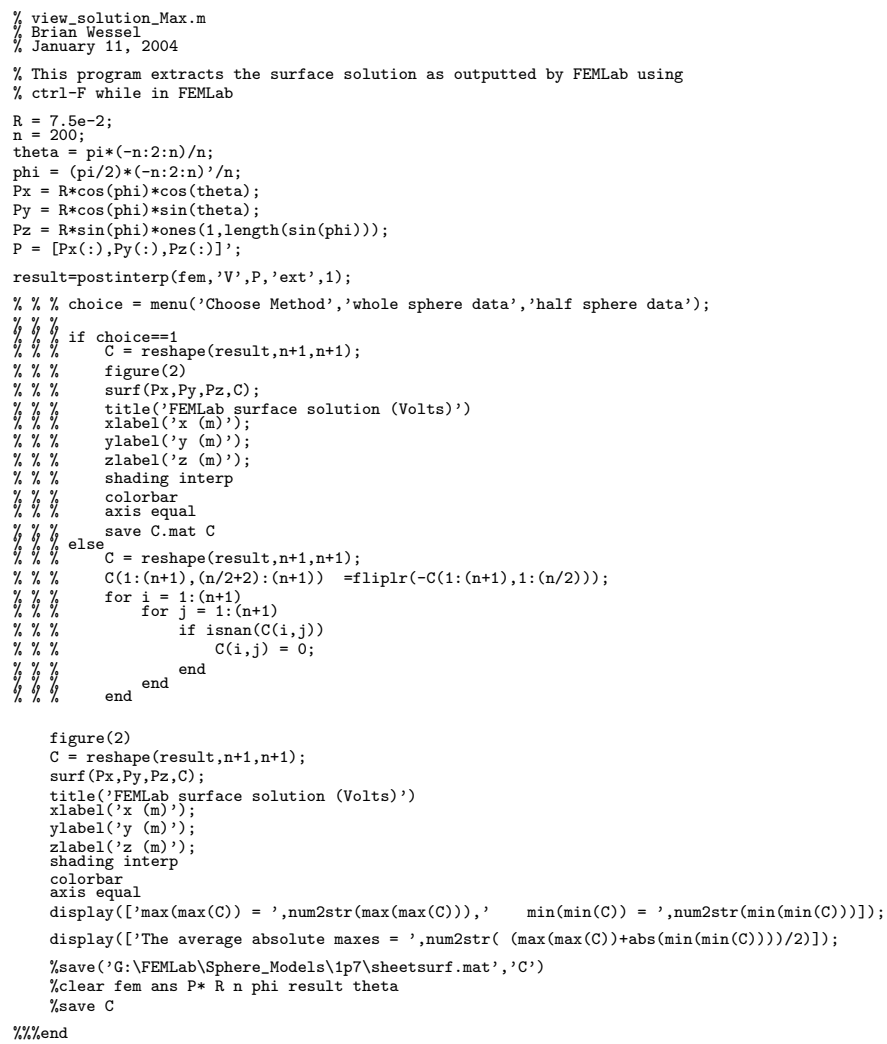




\section{.4 FOUR SHELL FILES}

Contains the following files:

- test_potential4shell_VII.m

- potential4shell.c 


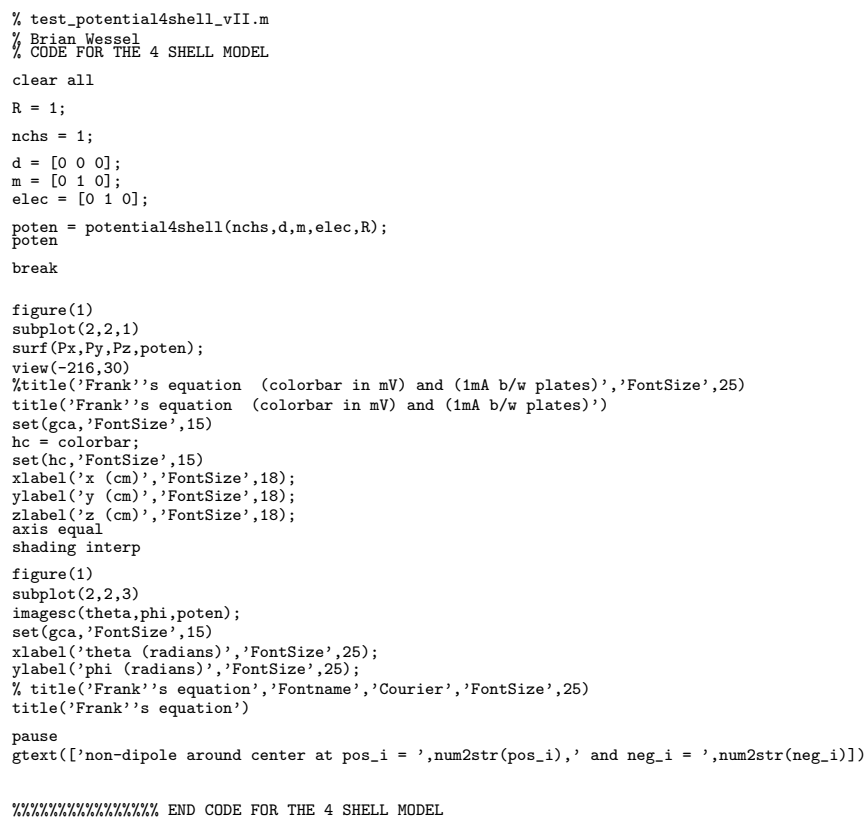




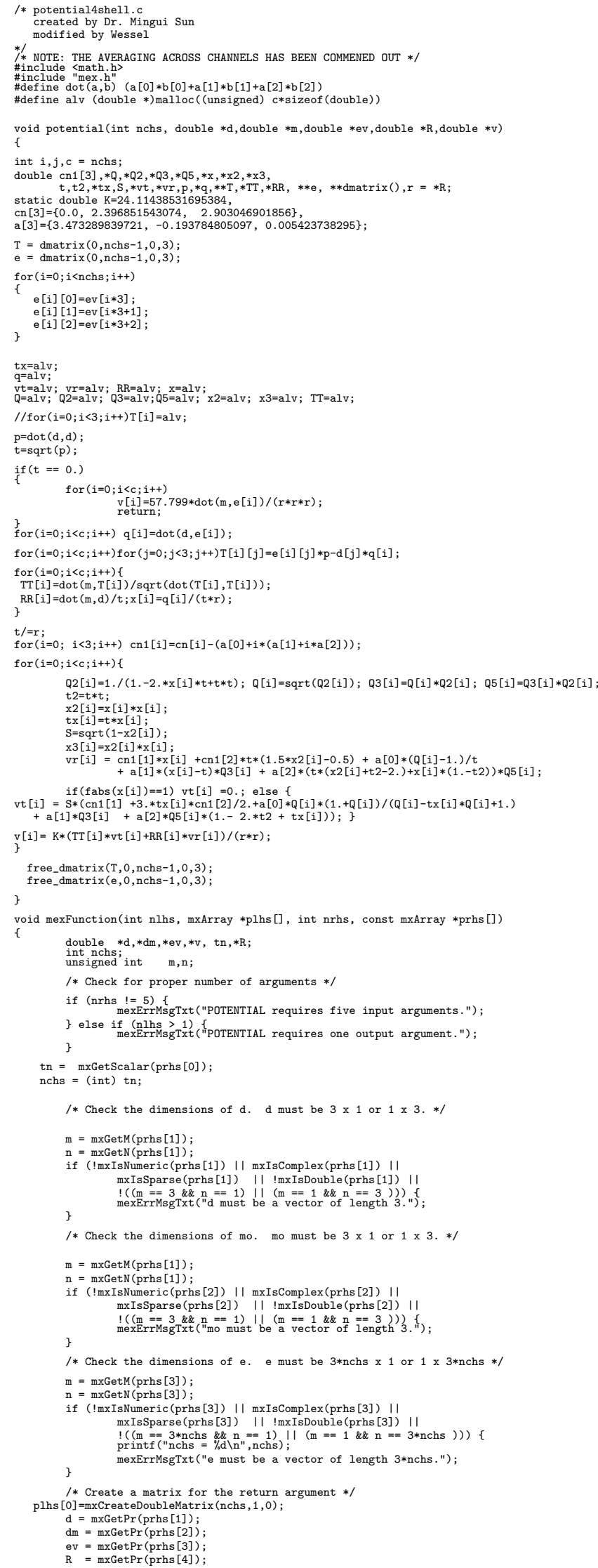




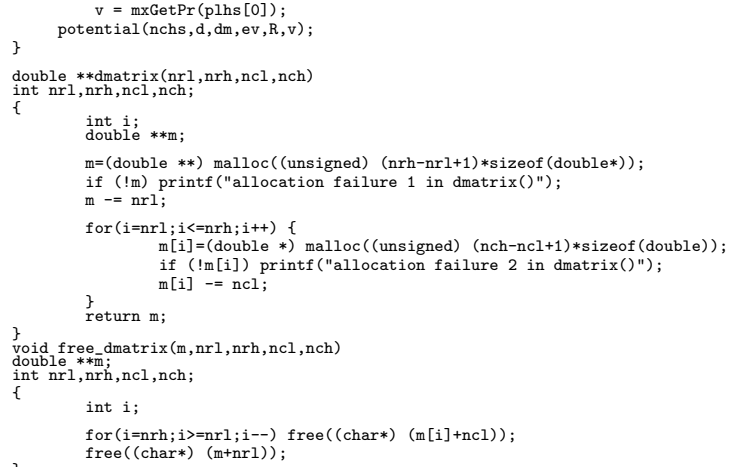




\section{.5 FE VS FRANK EQUATION}

Contains the following files:

- EF_FEM_Cond_DC_1shellmodelv3.m

- frankpotential.c (refer to .1) 


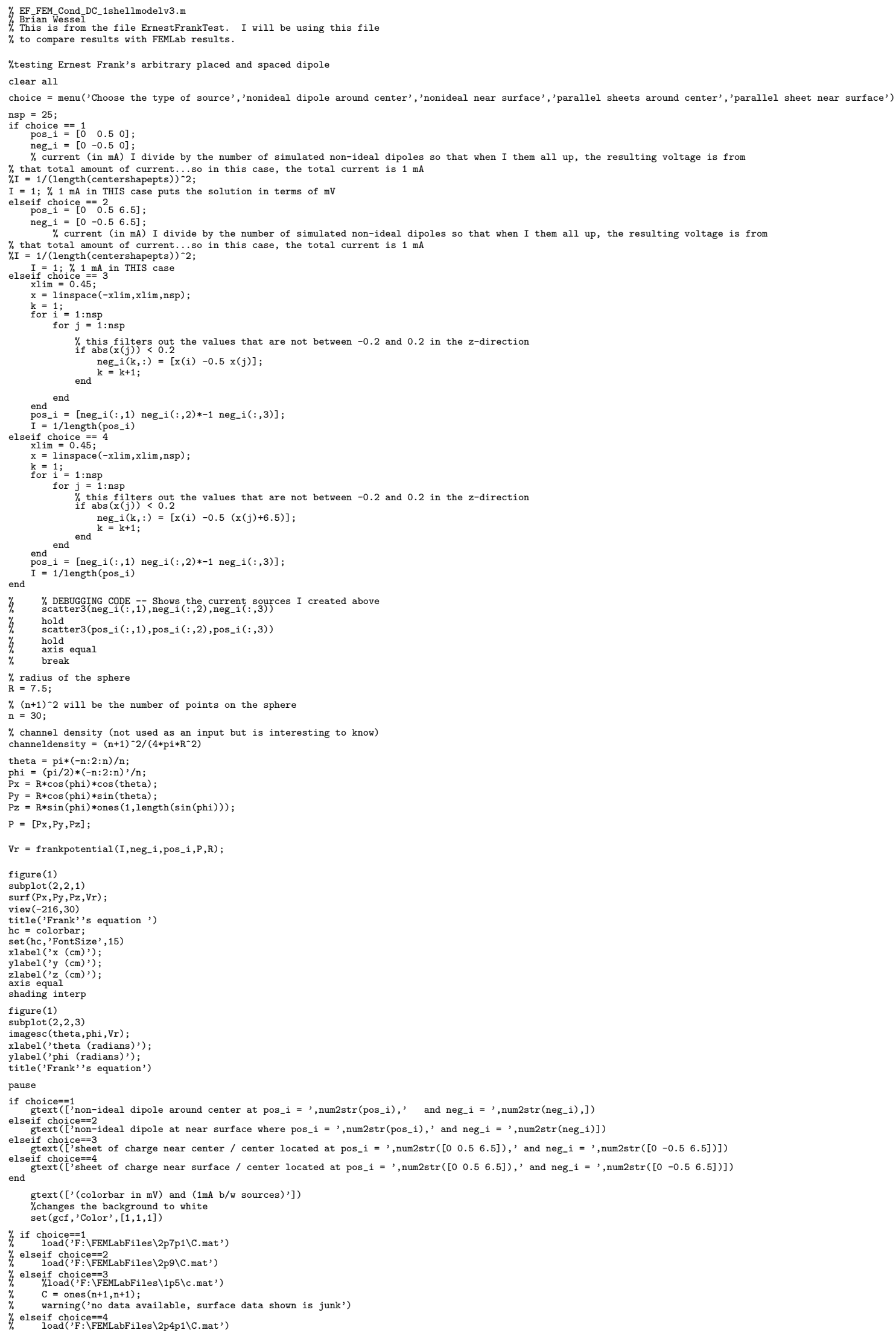




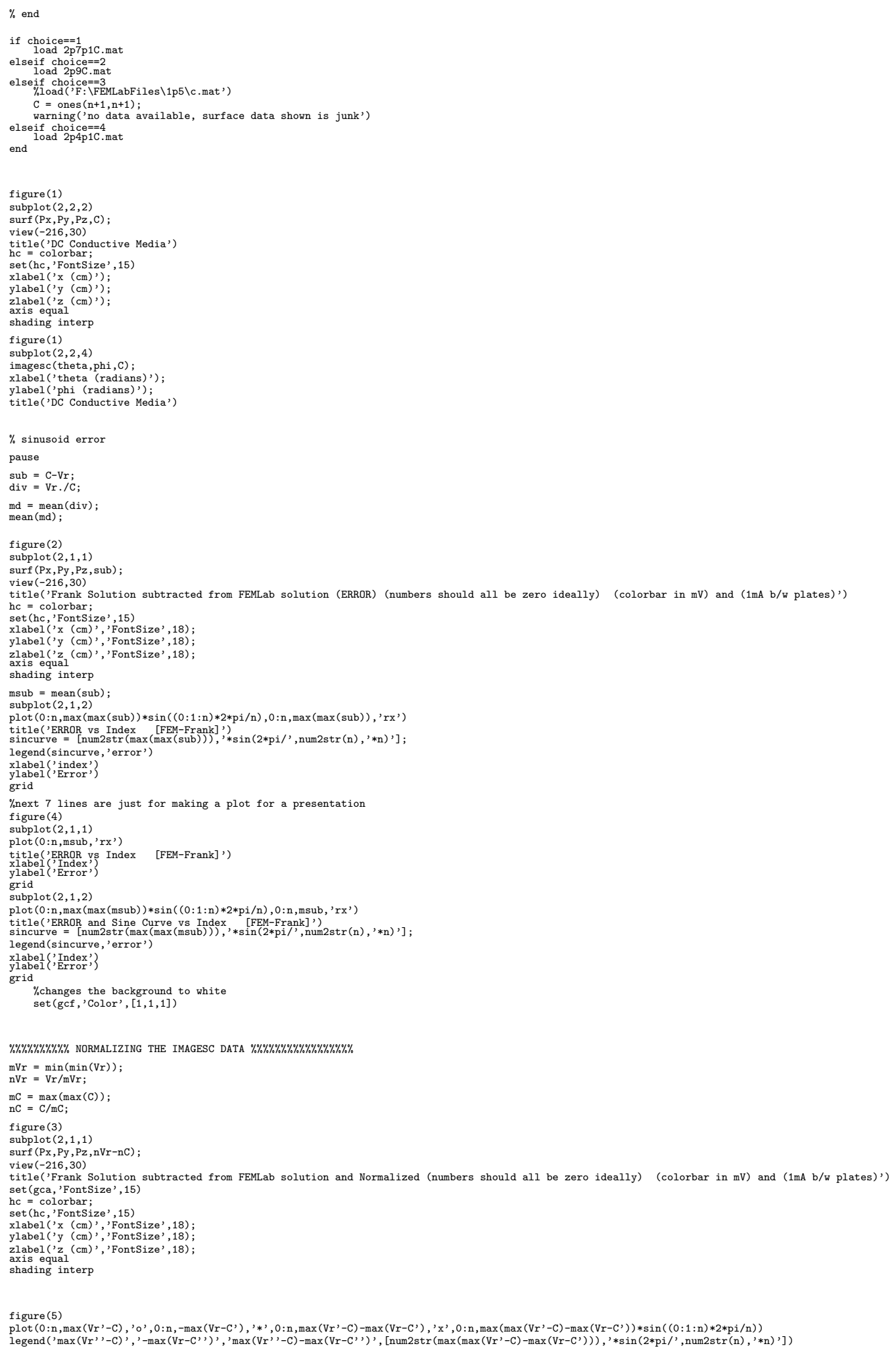




\section{.6 EXTRACTING POINTS FEMLAB OUTPUT (CTRL-F)}

Contains the following file:

- exps.m 


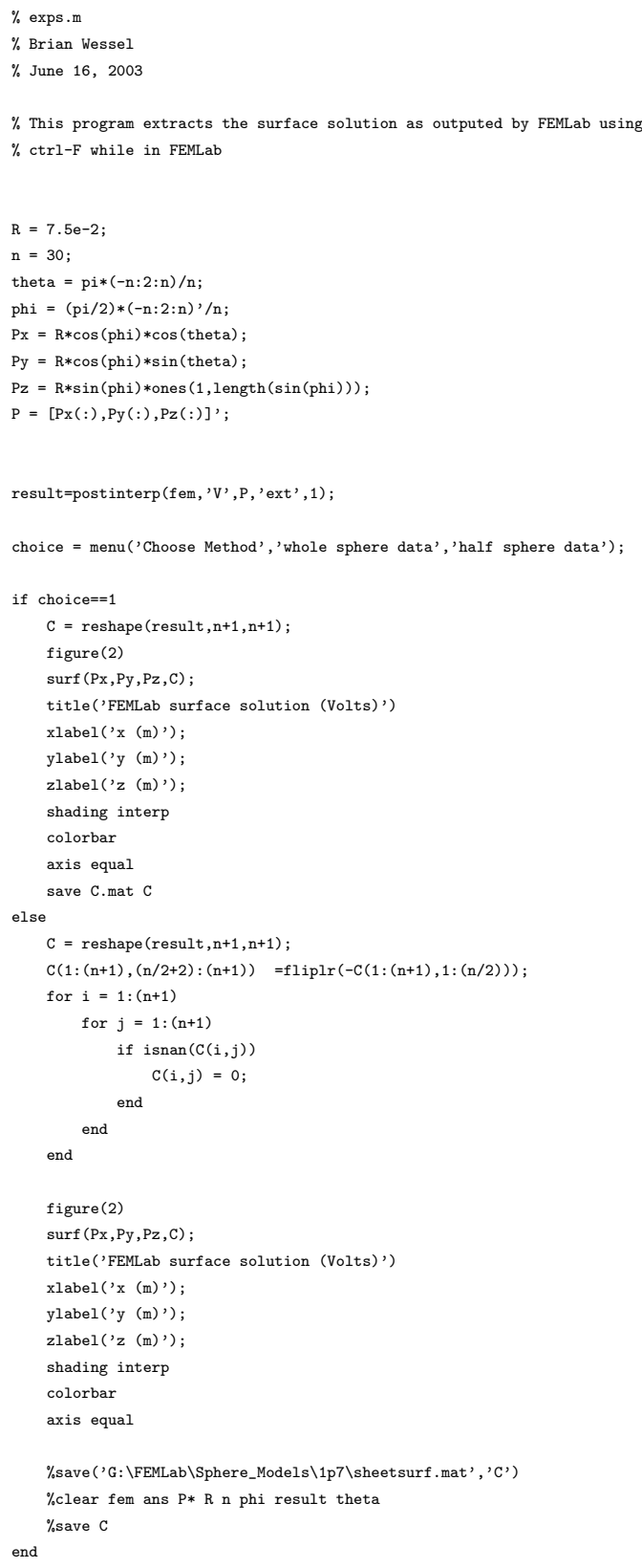




\section{.7 IDEAL VS NON-IDEAL DIPOLES $(2 \mathrm{D} \infty \mathrm{MEDIUM})$}

Contains the following file:

- ContourPlotsV2.m 


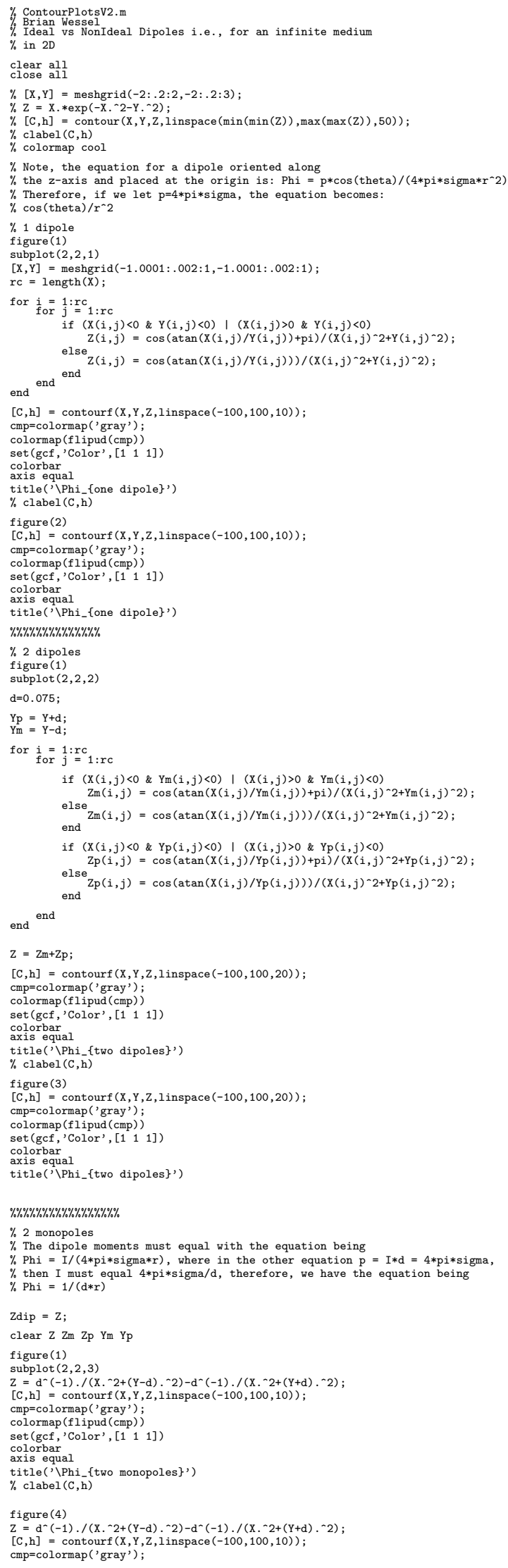


colormap (flipud (cmp))

set (gcf, 'Color', $\left.\left[\begin{array}{lll}1 & 1 & 1\end{array}\right]\right)$

colorbar

title('\Phi_\{two monopoles\}')

$\%$ error between 2 dipoles and two monopoles

Zerr $=$ Z-Zdip;

figure (1)

$[\mathrm{C}, \mathrm{h}]=\operatorname{contourf}(\mathrm{X}, \mathrm{Y}, \mathrm{Zerr}, \mathrm{linspace}(-100,100,10))$

cmp $=$ colormap ('gray')

colormap (flipud (cmp))
set (gcf, 'Color', [ $\left.\begin{array}{lll}1 & 1 & 1\end{array}\right]$ )

$\%$ clabel (C,h)

title('Error --> (\Phi_\{monopoles\}-〈Phi_\{dipoles\})')

figure (5)

$[\mathrm{C}, \mathrm{h}]=\operatorname{contourf}(\mathrm{X}, \mathrm{Y}, \mathrm{Zerr}$, linspace $(-100,100,10))$

cmp $=$ colormap ('gray');

colormap (flipud (cmp))

set (gcf, 'Color', [ [ [ 1111$]$ )

axis equal

title('Error --> (\Phi_\{monopoles\}--Phi_\{dipoles\})') 


\section{.8 IDEAL VS NON-IDEAL DIPOLES (3D SPHERICAL MEDIUM)}

Contains the following files:

- ErnestFrankTestv2.m

- frankpotential.c (refer to .1)

- potential.c (refer to .1) 


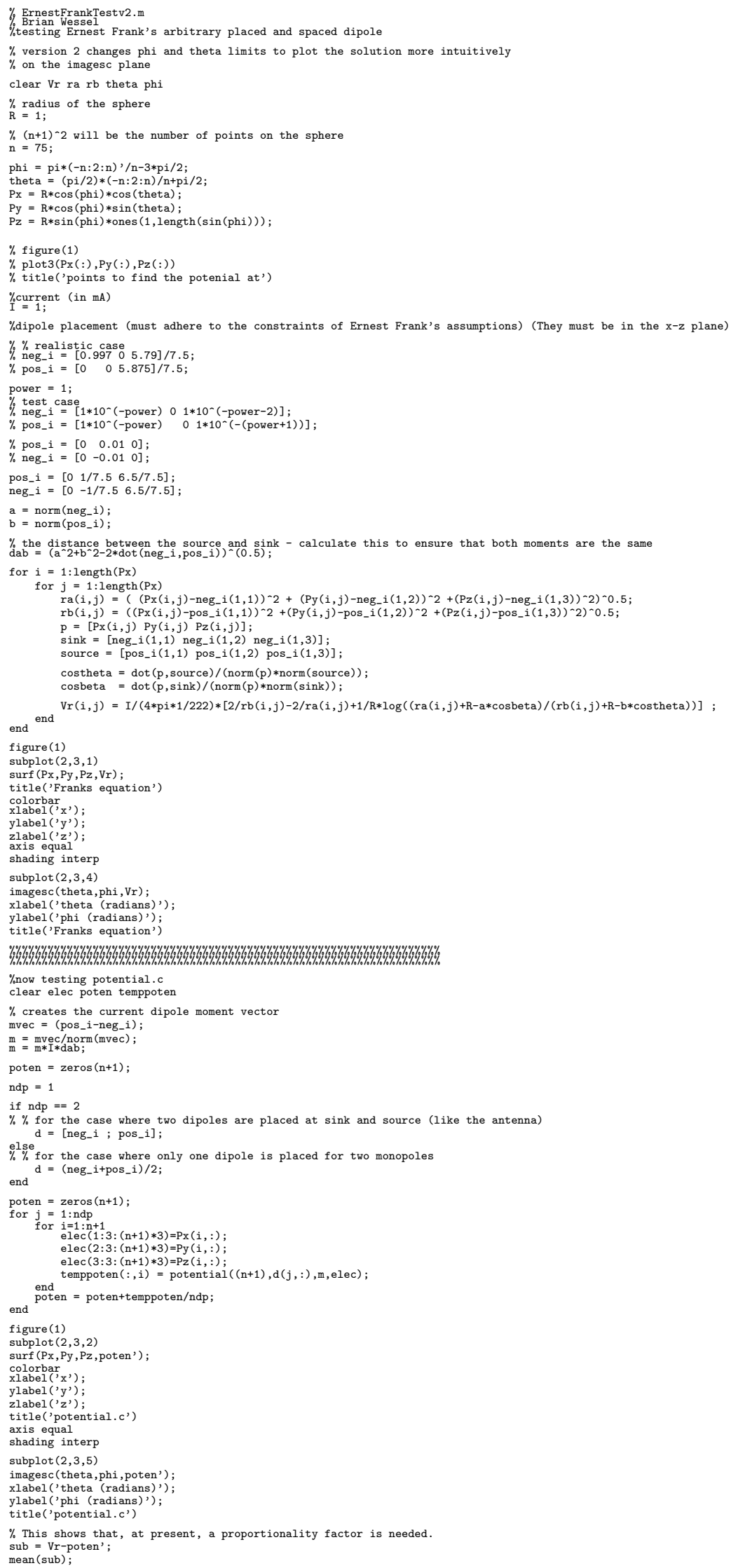


$\max ($ sub) ;

\% "format rat" ensures that if the numbers in sub are all approximately the same thing, it can display it as such and not just the differences $\% \%$ in the 10 th

\% format rat

surf (Px, Py, Pz, sub);

shading interp

xlabel (' $x$ ');

ylabel ('y')

title('Error (Vfrank-Videal)',

axis equal
colorbar

$\operatorname{subplot}(2,3,6)$

imagesc (theta, phi, sub);

xlabel ('theta (radians)');

ylabel('phi (radians)');

$\% \% \% \% \% \% \% \% \% \% \% \% \% \% \% \% \% \%$ Adding the dB interpretation $\% \% \% \% \% \% \% \% \% \% \% \% \% \% \% \% \% \% \% \%$

$\% \% \%$ This isn't working well, because I get $\log$ (zero) which is undefined

$\% \% \%$ so it might be a better idea to just use percent difference

$\mathrm{DB}=20 * \log 10(\operatorname{abs}(\operatorname{sub} / \max (\max (\mathrm{Vr}))))$

figure (2)

surf (Px, $\mathrm{Py}, \mathrm{Pz}, \mathrm{DB})$

shading interp

xlabel $\left(x^{\prime}\right)$;

ylabel('y');

zlabel(' $z$ ');
title('dB Error --> 20*1og $10(\mid$ Verror $\mid / \max (\max (\operatorname{Vr})))$ ')

title ('dB

axis equal
colorbar

subplot $(2,1,2)$

imagesc (theta, phi, DB);

ylabel('phi (radians)');

title('dB Error')

figure (3)

$\mathrm{DB}=20 * \log 10(\mathrm{abs}(\mathrm{sub} . / \mathrm{Vr}))$

figure (2)

subplot $(2,1,1)$

surf $(\mathrm{Px}, \mathrm{Py}, \mathrm{Pz}, \mathrm{DB})$

xlabel (', '

ylabel ('y');

title('dB Error --> 20*1og $10(\mid$ Verror $\mid . / V r)$ ')

axis equal

subplot $(2,1,2)$

imagesc (theta, phi, DB)

Ylabel('phi (radians)')'

title('dB Error')

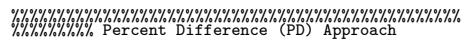

$\mathrm{PD}=\mathrm{abs}(\mathrm{sub} . /(\mathrm{Vr}+1 \mathrm{e}-8)) * 100 ;$

figure (4)

subplot $(2,1,1)$

surf (Px, Py, Pz, $P D)$
shading interp

xlabel (' $x$ ')

ylabel('y');

zlabel(' $z$ ');

title ('Percent Difference --> |Verror $\mid / \max (\max (\mathrm{Vr})) * 100$ ')

colorbar

subplot $(2,1,2)$

a,phi, PD)

ans)'

title('Percent Difference')

$\%$ just a separate plot so I can put in Thesis

figure (5)
$\operatorname{surf}(\mathrm{Px}, \mathrm{Py}, \mathrm{Pz}, \mathrm{PD})$

shading interp

xlabel ('x (cm)', 'FontSize', 15)

ylabel ('y (cm)', 'rontSize', 15)

\%title('Percent Difference --> Error./FrankSolution*100', 'FontSize', 15)

axis equal

colorbar
view (-55,
grid off

set (gca,'FontSize', 15)

cmp $=$ colormap (' autumn')

title('Percent Error') 


\section{.9 HYPOTHETICAL ANGLE}

Contains the following files:

- shapesoptimize_sym_TEST_PSI.m

- shapestotarclength.m (refer to .2)

- shapesfundersq.m (refer to .1)

- shapesptsopt_v2.m (refer to .2)

- frankpotential.c (refer to .1) 


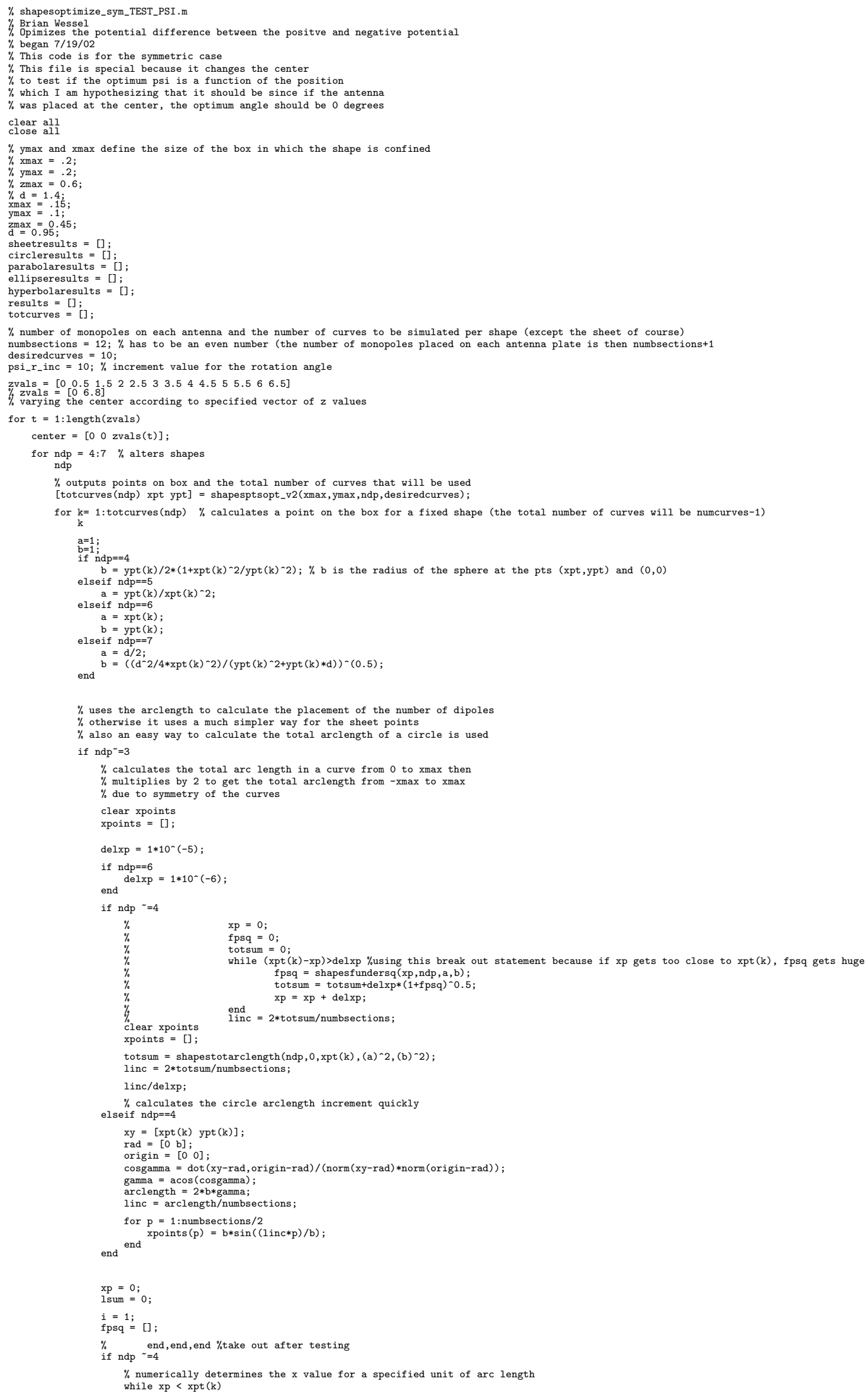


while 1 sum $<1$ inc
fpsq $=$ shapesfundersq (xp, ndp, a, b);

1 sum $=1$ sum $+\operatorname{delxp} *(1+f p s q) \sim 0.5$;

$\mathrm{xp}=\mathrm{xp}+\operatorname{delxp}$
if $\mathrm{xp}>\mathrm{xpt}(\mathrm{k})$

end end

lsum $=0$;

if $\mathrm{xp}<\operatorname{xpt}(\mathrm{k})$

end $x p o i n t s(i)=x p$

$i=i+1$;

end xpoints $=[$ xpoints, $\operatorname{xpt}(k)]$

else ndp $==3$

linc $=\operatorname{xpt}(\mathrm{k}) * 2 /$ numbsections $;$

end $\mathrm{xp}$

$\%$ creates negative values and adds a zero point for the xpoints

$\%$ nxpoints = -xpoints;

(-xpoints) 0 xpoints];

gth (xpoints)

zpoints $=-$ zmax $:$ linc: $:$ max;

ypoints $=[] ;$

$\%$ calculates ypoints according to which shape the user specifies.

ypoints $=$ zeros (length $($ xpoints $), 1)$;

elseif ndp $==4 \quad \%$ circle points

ypoints $=\operatorname{abs}\left(\left(b^{\wedge} 2-(x p o i n t s \cdot * x p o i n t s)\right) \cdot \wedge(0.5)-b\right)$

elseif ndp $==5 \%$ parabola pts

ypoints $=\mathrm{a} *$ (xpoints.$*$ xpoints $)$;

elseif ndp $==6 \%$ ellipse points

ypoints $=\operatorname{abs}\left(\left(b^{\wedge} 2-b^{\wedge} 2 / a^{\wedge} 2 *(\right.\right.$ xpoints.$*$ xpoints $\left.\left.)\right) \cdot{ }^{\wedge}(0.5)-b\right) ;$

elseif ndp $==7 \quad \%$ hyperbola points end

ndp $==7 \quad \%$ hyperbola points
oints $=\left(a^{\wedge} 2+a^{\wedge} 2 / b^{\wedge} 2 *(\right.$ xpoints.$\left.* x p o i n t s)\right) \cdot{ }^{\wedge}(0.5)-a ;$

$\%$ hold on, scatter(xpoints, ypoints),

$\%$ \% or $i=1:$ length (xpoints), $\operatorname{arcl}(i)=$ shapestotarclength (ndp, $\left.0, x p o i n t s(i), a^{\wedge} 2, b^{\wedge} 2\right)$; , if $i>1$, figure $(10), p l o t\left(\operatorname{arcl}(i)-\operatorname{arcl}(i-1), i, 0^{\prime}\right)$, hold on, end, end,

$\%$ end, end \% \% delimit this code after testing

shapepts $=[] ;$

for $j=1:$ length (zpoints)

w $=1$ : length (xpoints)
shapepts $=[$ shapepts; $[x p o i n t s(w)$ ypoints $(w)$ zpoints $(j)]] ;$

end

[shopes; [xpoints(w) ypoints(w) zpoints $(j)]$;

$\%$ bothshapepts $=[$ shapepts; negshapepts $] ;$

$\%$ figure(1)

$\%$ scatter3 (bothshapepts (: , 1), bothshapepts (: , 2), bothshapepts (: , 3));

$\%$ xlabel(' $x$ ');

$\%$ ylabel(' 'y');

$\%$ zlabel ('z');
$\%$ axis equal

for psi_r $=0$ :psi_r_inc: $90 \%$ rotates psi_r for a fixed shape and curvature

$\%$ [points] =shapesintersect () should go here if $I$ end up wanting to use the assymetric case

psi_r

rshapepts $=$ shapepts

rnegshapepts $=$ negshapepts

$\%$ rotate the antenna

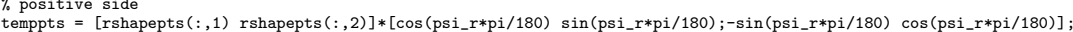

rempts $=$ rshapepts $(:, 1)=$ temppts $(:, 1) ;$
rshapepts $(:, 2)=$ temppts $(:, 2) ;$

$\%$ negative side

temppts $=[$ rnegshapepts $(:, 1)$ rnegshapepts $(:, 2)] *\left[\cos \left(p s i \_r * p i / 180\right)-\sin \left(p s i \_r * p i / 180\right) ; \sin \left(p s i \_r * p i / 180\right) \cos \left(p s i \_r * p i / 180\right)\right] ;$

rnegshapepts $(:, 1)=$ tempts $(:, 1)$
rnegshapepts $(:, 2)=$ temppts $(:, 2)$

phi_r $=$ pi/2;

phi_r $=$ pi
$\%$ pos side

temppts $=[$ rshapepts $(:, 3)$ rshapepts $(:, 1)] *[\cos ($ phi_r $r) \sin ($ phi_r $) ;-\sin ($ phi_r $) \cos ($ phi_r $)]$

rshapepts $(:, 3)=\operatorname{temppts}(:, 1)$
$\operatorname{rshapepts}(:, 1)=\operatorname{temppts}(:, 2)$

\% neg side

temppts $=$ rnegshapepts $(:, 3)$ rneg
rnegshapepts $(:, 3)=$ tempts $(:, 1) ;$
regshapepts $(:, 1)=$ temppts $(:, 2)$

bothshapepts $=[\operatorname{rshapepts}(:, 1) \operatorname{rshapepts}(:, 2)+d / 2 \operatorname{rshapepts}(:, 3)+\operatorname{center}(1,3) ; \operatorname{rnegshapepts}(:, 1) \operatorname{rnegshapepts}(:, 2)-d / 2 \operatorname{rnegshapepts}(:, 3)+\operatorname{center}(1,3)]$ $\%$ bothshapepts $=[$ shapepts; negshapepts $]$

$\%$ sets the data within bounds and checks that the farthest point is not touching or outside the sphere

temppts $=[] ;$
for $\mathrm{u}=1:$ length (bothshapepts)

$\operatorname{MaxR}(\mathrm{u})=\operatorname{norm}($ bothshapepts $(\mathrm{u},:))$;

if abs (bothshapepts $(u, 2))<(y \max +d / 2) \&$ abs $($ bothshapepts $(u, 3))>(\operatorname{center}(1,3)-x \max ) \& \operatorname{abs}($ bothshapepts $(u, 3))<(\operatorname{center}(1,3)+x \max )$

$\%$ temppts = [temppts; bothshapepts $(u,:)]$;

敞R $=\max (\operatorname{MaxR})$

MaxR $=\max (\operatorname{MaxR}) ;$
$\%$ bothshapepts $=$ temppts;

$\%$ figure(3)

$\%$ scatter3 (bothshapepts (: , 1), bothshapepts $(:, 2)$, bothshapepts $(:, 3))$

$\%$ xlabel (' $x$ ')

$\%$ ylabel ('y')

$\%$ zlabel (' $z$ ');

$\%$ view $(-90,0)$;

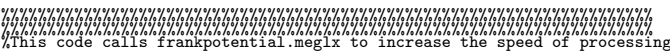




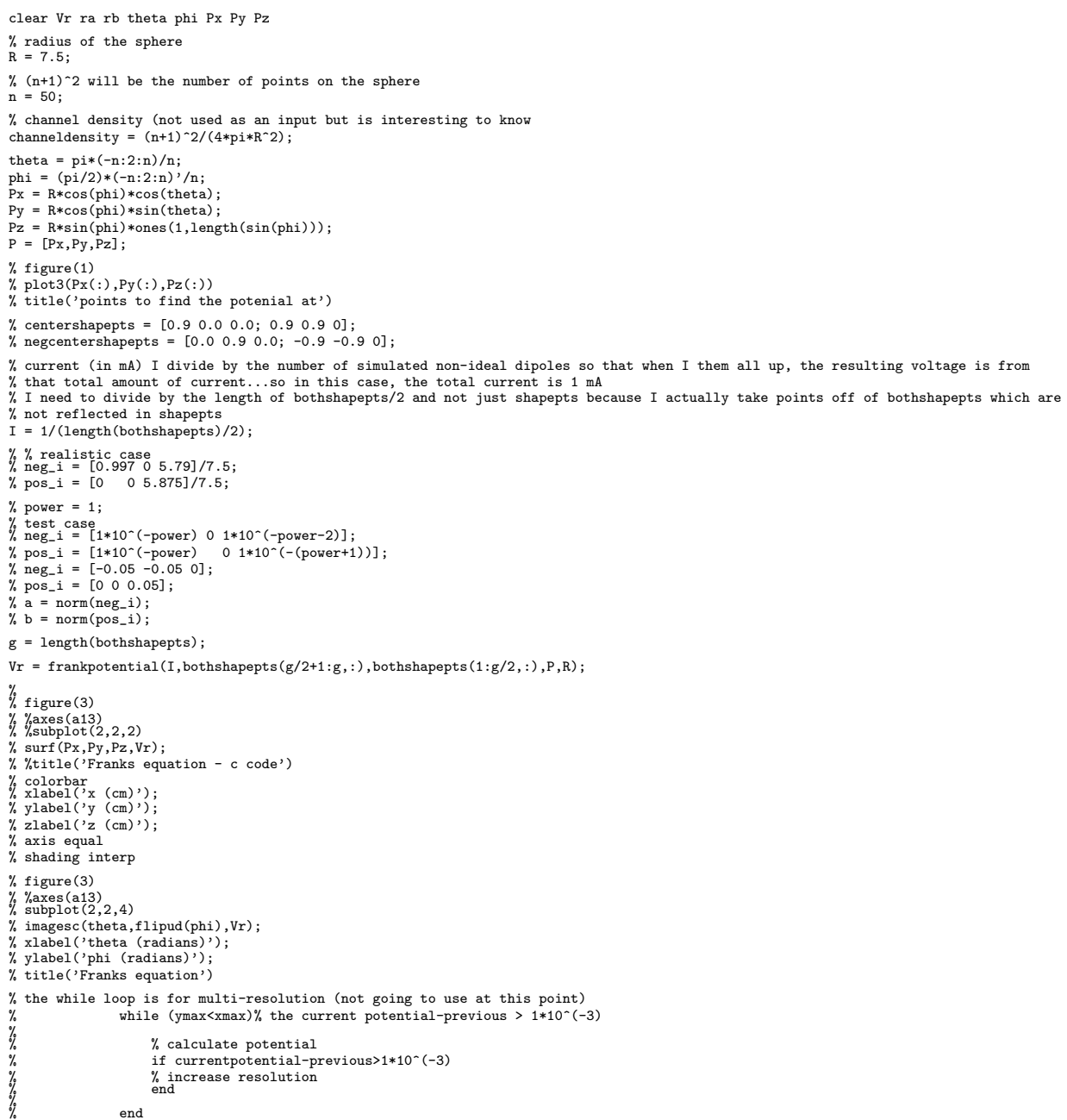

results $=[$ results $; \max (\max (V r)) \min (\min (V r))$ ndp psi_r $\mathrm{k}$ a b MaxR $x \max y \max z \max d$ numbsections desiredcurves center $(1,3)]$ end

path1 $=\left[{ }^{\prime} \mathrm{C}: \backslash\right.$ Documents and Settings $\backslash$ bwessel $\backslash$ Desktop $\backslash$ PsiTest $\backslash \operatorname{Trial1} \backslash$ results' ${ }^{\prime}$, num2str $(z v a l s(t)),{ }^{\prime} \cdot$ dat $\left.^{\prime}\right]$

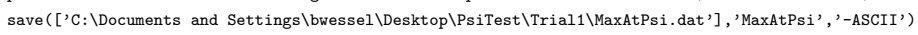

save (path1, 'results', '-ASCII')

\% $\quad \operatorname{save(['/net/bwessel-lh/usr3/users/bwessel/matlab/Results/PsiTest/results',~num2str(zvals(t)),~'.dat~'],~'results',~,~-ASCII')~}$

$\%$ save resultsX_parab_ell_hyp.dat results -ascii

load results.dat

results $=[]$;

end \% end loop to iterate center position

for $\mathrm{t}=1:$ length (zvals)

['results', num2str (zvals(t)), '.dat']

results $=1$ oad $\left(\right.$ path $1,{ }^{\prime} \cdot$ dat $\left.^{\prime}\right]$ )

for $j=1$ :length (results)

$f \operatorname{results}(j, 3)==3$

$s=[$ sheetresults; results $(j,:)]$

lseif $\operatorname{results}(j, 3)==4$

[circleresults;results $(j,:)$;

parabolaresults $=[$ parabolaresults; $\operatorname{results}(j,:)]$

if $\operatorname{results}(j, 3)==6$

ellipseresults $=[$ ellipseresults $; \operatorname{results}(j,:)] ;$

hyperbolaresults $=[$ hyperbolaresults; $\operatorname{results}(j,:)]$;

end en 


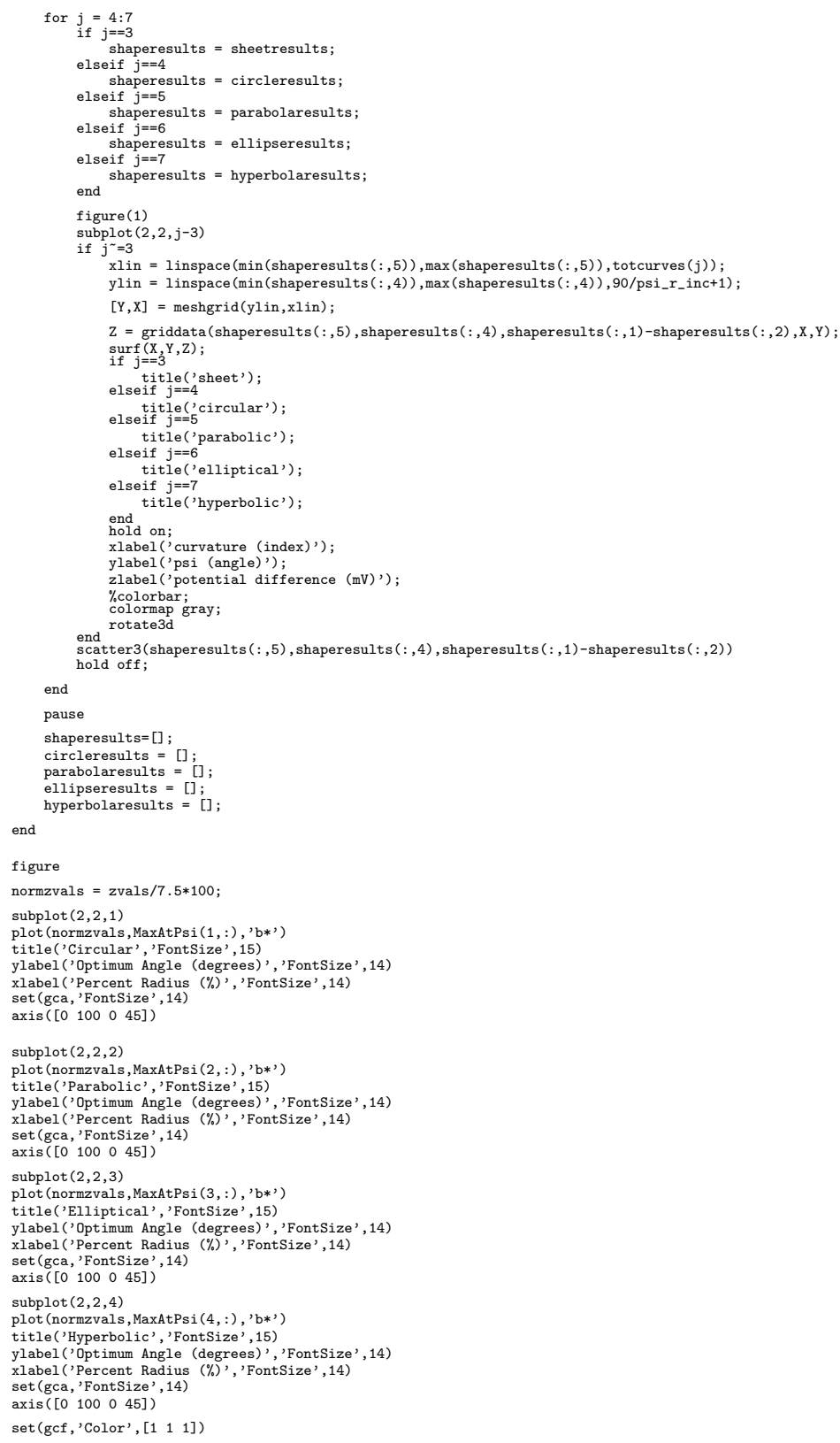




\section{.10 EXTRACTING POINTS FROM SLICES IN 3D}

Contains the following file:

- slicedata.m 


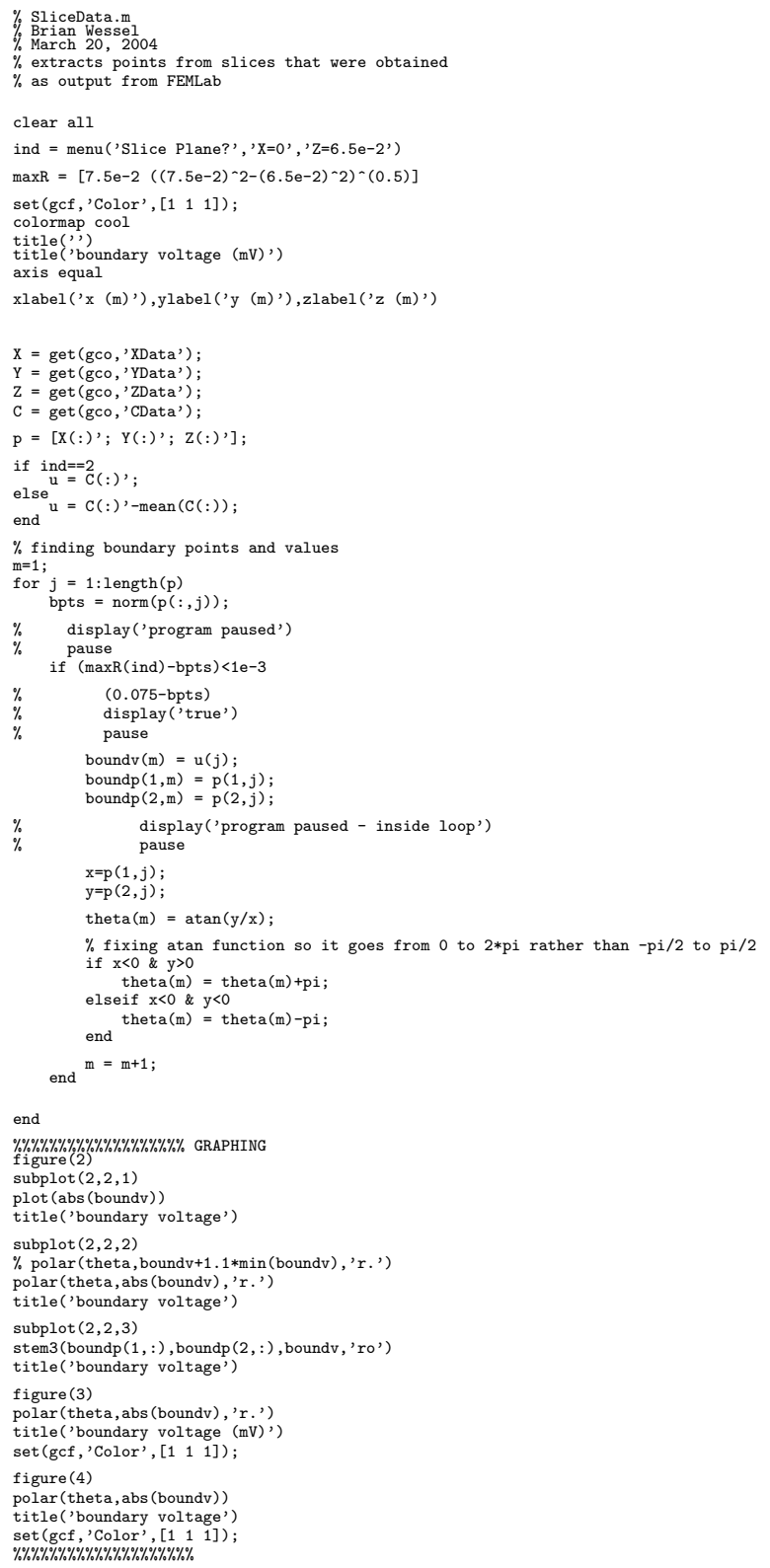




\section{.11 PDE TOOL CODE - EPOXY}

Contains the following files:

- create_antennas_noReflector.m

- commandline_v9.m

- shapesfundersq.m (refer to .1)

- shapestotarclength.m (refer to .2)

- shapesptsopt_v2.m (refer to .2)

- normalpointsv2.m (refer to .3) 


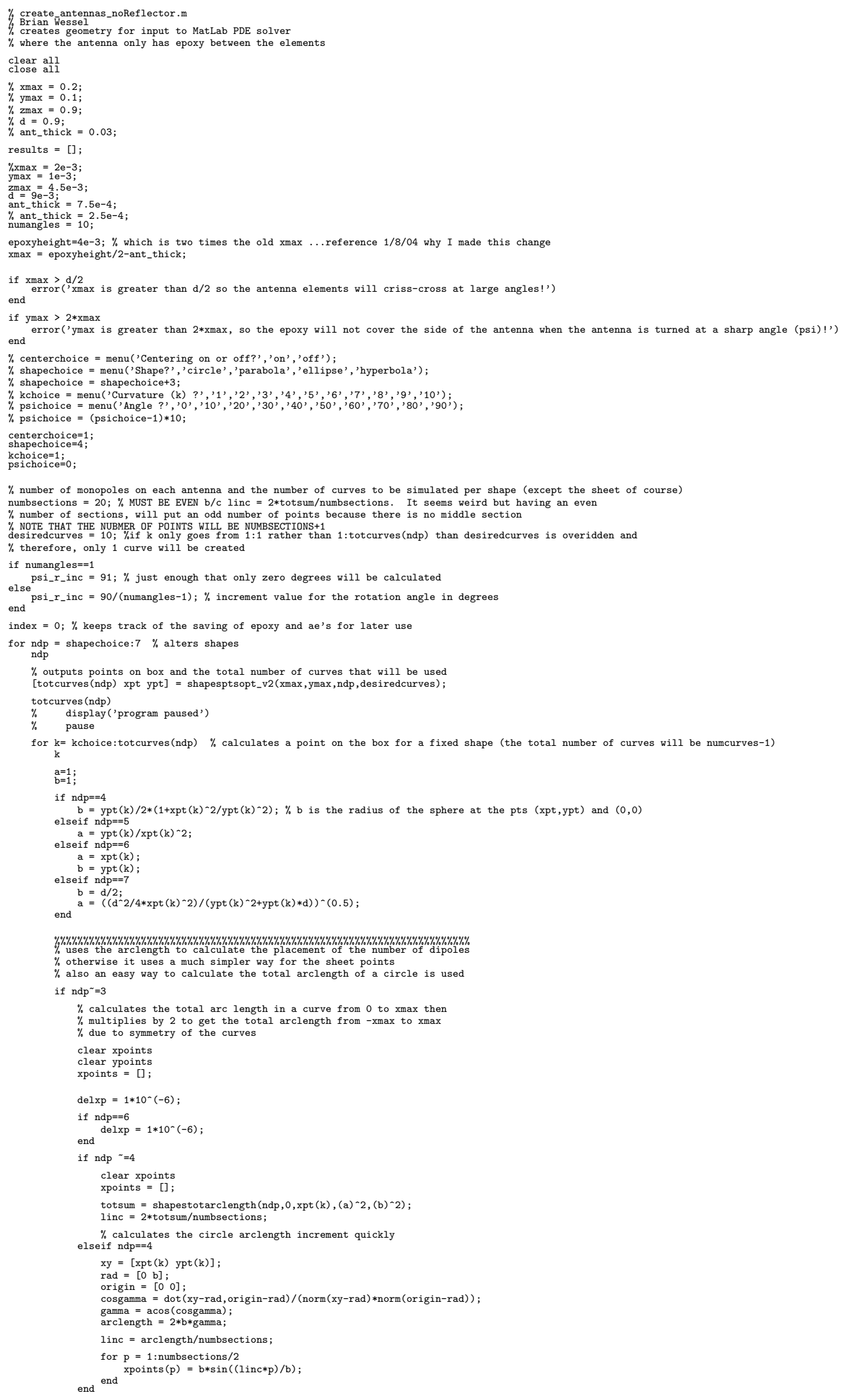




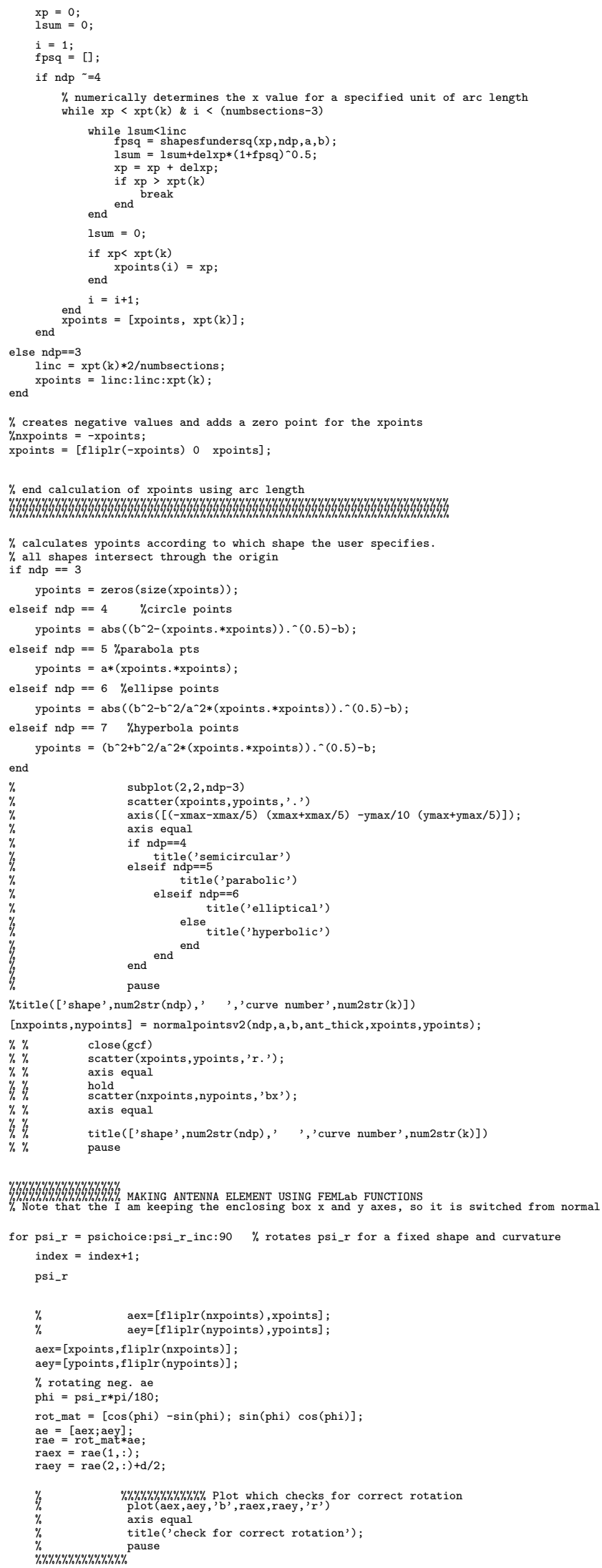




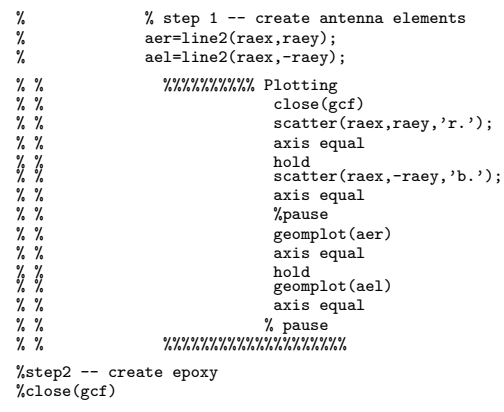

$\% \% \% \% \% \% \% \% \% \% \% \% \% \% \% \% \% \% \% \% \% \% \% \%$ code for creating outside antenna first . . uses output of normalptsv2 m m \%\%\%\%\%\%\%\%\%\%\%\%\%\%\%\%\%\%\%\%\%\%\%\%\%\%\% half $=$ raex (numbsections $+2:$ length (raex))

haf $=$ raey (numbsectionst $:$ length (raey)):

centerchoice $=1 \%$ This choice will center the antenna element within the epoxy

$\%$ the difference

$\%$ the difference between having a shield of epoxy vs free space and will give
$\%$ into how much a shield is effective at pushing the current to the far-field

$\%$ if psi_r $=0$, we don't want to add extra epoxy or it will cause an error because the points
$\%$ will be too close to connect with a line... however, I should add about half the antenna thickness

$\%$ to each side so that it doesn't make a sharp point near the tip of the antenna as in the case of the

$\%$ elliptically shaped antenna elements

$\begin{aligned} \text { psi_r } & ==0 \\ \text { xtra } & =\text { ant_thick } / 2\end{aligned}$

$\% \quad$ tra $=$ ant_thick $* 4 / 5$

-xtra) (halfx (length(halfx))-xtra) fliplr(halfx) (half $x(1)+x \operatorname{tra})$ (halfx(1)+xtra) ] epoxy $=1$ ine $2($ tot $x$, toty)

$\%$ (for old way \#1)this strange sin*blah is so that at large angles the epoxy does not overlap itself and

$\%$ old way \#1 -- xtra $=$ half $x(1,1)+\sin ($ psi_r*pi/180)*(ymax+ant_thick);

$\%$ old way $\# 1--x$ xtra $=$ half $x(1,1)+\sin (p s i x ;$
$\%$ old way $\# 2--x \operatorname{xtra}=\max ($ raex $)+1 / 5 * x \max ;$

$\%$ xtra $=\min ($ raex $)+2 * x \max +$ ant_thick;

halfy $=$ fliplr(halfy)

widthant $=\max ($ half $\mathrm{x})$-halfx (1)

$\% \quad$ xout $=$ ant_thick $* \cos (\mathrm{phi})$

xout $=$ ant_thick $* \cos ($ phi $) * 3 / 5$;

$\% \quad$ xtra $=($ epoxyheight-widthant $) / 2$

xtra $=$ (epoxyheight-widthant) $* 4 / 5$

yout $=$ ant_thick $* \sin (\mathrm{phi})$

$\%$ totx $=[$ halfx $\max ($ half $x)(\max ($ half $x)+x t r a)(\max ($ half $x)+x t r a) \max ($ half $x)$ fliplr(halfx) halfx(1) (halfx(1)-xtra) (halfx(1)-xtra) halfx(1)];

$\% \%$

$\% \%$

toty $=$ [halfy (halfy (length

$\% \% \%$ Sophisticated
totx $=$ halfx
toty $=$ halfy

$\% \quad \operatorname{totx}=[\operatorname{totx}(\max ($ half $x)+x o u t)]$

totx $=[$ totx (halfx $($ length $($ half $x))+x$ out $)] ;$
toty $=[$ toty (halfy $($ length(halfy) + yout $)] ;$

totx $=[$ totx (max (half $x)+$ xout $+x t r a))$
toty $=[$ toty $($ halfy $($ length $($ halfy $))+$ yout $)]$

totx $=[$ totx $(\max ($ half $\mathrm{f})+\mathrm{xout}+\mathrm{xtra})] ;$
toty $=[$ toty $-($ halfy $($ length $($ halfy $))+$ yout $)] ;$

$\% \quad \operatorname{totx}=[\operatorname{totx}(\max ($ halfx $)+x$ out $)] ;$

totx $=[$ totx $($ half $x($ length $($ half $x))+$ xout $)] ;$
toty $=[$ toty $-($ halfy $($ length $($ half $y))+$ yout $)]$

totx $=[$ totx fliplr(half $x)] ;$

totx $=[$ totx (halfx (1)-xout) $] ;$

totx $=[\operatorname{tot} x($ halfx $(1)-x$ tra- $x$ out $)]$

$\begin{aligned} \text { totx } & =[\text { totx }(\text { halfx }(1)-x \text { tra-xout })] \\ \text { toty } & =[\text { toty (half }(1)-\text { yout })] ;\end{aligned}$

totx $=[$ totx (halfx (1)-xout) $] ;$
toty $=[$ toty (halfy (1)-yout) $] ;$

\begin{tabular}{|c|c|}
\hline$\% \% \%$ & Primitive Modeling \\
\hline$\% \%$ & $\begin{array}{l}\operatorname{totx}=[(\max (\text { half } x)+x \operatorname{tra} a)] \\
\text { toty }=[\min (\text { halfy })-\text { ant_thick } / 2] ;\end{array}$ \\
\hline$\% \%$ & $\begin{array}{l}\operatorname{totx}=[\operatorname{totx}(\max (\text { half } x)+x \operatorname{tra})] ; \\
\text { toty }=[\text { toty } \max (\text { halfy })] ;\end{array}$ \\
\hline$\% \% \%$ & $\begin{array}{l}\operatorname{totx}=[\text { totx }(\max (\text { half } x)+x \text { tra+ant_thick })] ; \\
\text { toty }=[\text { toty } \max (\text { halfy })] ;\end{array}$ \\
\hline$\% \%$ & $\begin{array}{l}\operatorname{totx}=[\operatorname{totx}(\max (\text { half } x)+x \text { tra+ant_thick })] ; \\
\text { toty }=[\text { toty }-\max (\text { halfy })] ;\end{array}$ \\
\hline$\% \% \%$ & $\begin{array}{l}\operatorname{totx}=[\text { totx }(\max (\text { half } x)+x \operatorname{tra})] \\
\text { toty }=[\text { toty }-\max (\text { halfy })] ;\end{array}$ \\
\hline$\% \%$ & $\begin{array}{l}\operatorname{totx}=[\operatorname{totx}(\max (\text { half } x)+x \operatorname{tra})] ; \\
\text { toty }=[\text { toty }-(\min (\text { halfy })-\text { ant_thick } / 2)] ;\end{array}$ \\
\hline$\% \% \%$ & $\begin{array}{l}\operatorname{totx}=[\operatorname{totx}(\text { halfx }(1)-x \operatorname{xtra})] ; \\
\text { toty }=[\text { toty }-(\min (\text { halfy })-\text { ant_thick } / 2)] ;\end{array}$ \\
\hline$\% \%$ & $\begin{array}{l}\operatorname{tot} x=[\operatorname{totx}(\text { halfx }(1)-x \operatorname{tra})] ; \\
\text { toty }=[\text { toty }(\min (\text { halfy })-\text { ant_thick } / 2)] ;\end{array}$ \\
\hline
\end{tabular}

end 
else

$\%$ if psi_ $r=0$, we don't want to add extra epoxy or it will cause an error because the points

$\%$ will be too close to connect with a line
if $p$ pi $r=0$

totx $=[f \operatorname{liplr}($ half $x)$ half $x] ;$

else $\%$ (for old way \#1) this strange sin*blah is so that at large angles the epoxy does not overlap itself and

$\%$ therefore cause a probl

$\%$ old way $\# 1--$ xtra $=$ half $x(1,1)+\sin ($ psi_r*pi/180)*(ymax+ant_thick $)$

$\%$ old way $\# 2--\mathrm{xtra}=\max ($ raex $)+1 / 5 * \max$;

$\%$ xtra $=\min ($ raex $)+2 * x \max +$ ant_thick

xtra $=\min ($ raex $)+2 * x \operatorname{xmax}$

xtra xtra];

end

(fliplr(halfy) -halfy -halfy(1, length(halfy)) halfy(1,length(halfy))];

end $\% \% \% \% \% \% \% \% \% \% \% \% \% \% \% \% \% \% \% \% \% \% \% \%$ end create outside antenna first $\% \% \% \% \% \% \% \% \% \% \% \% \% \% \% \% \% \% \% \% \% \% \% \% \% \% \%$

$\% \% \% \% \% \% \% \% \% \% \% \% \% \% \% \% \% \% \% \% \% \% \% \%$ code for creating inside antenna first. . uses output of normalptsv4 m $\% \% \% \% \% \% \% \% \% \% \% \% \% \% \% \% \% \% \% \% \% \% \% \% \%$

$\%$ halfx $=$ raex (1:numbsections +1$) ;$

$\%$ if centerchoice $=1 \%$ This choice will center the antenna element within the epoxy

$\%$ This regime is fundamentally different from noncentering because it should expose

$\%$ the difference between having a shield of epoxy vs free space and will give insight

$\%$ into how much a shield is effective at pushing the current to the far-field

$\%$ if psi_r $=0$, we don't want to add extra epoxy or it will cause an error because the points

$\%$ will be too close to connect with a line
if $p s i \_r=0$

totx $=[$ fliplr(half $\mathrm{x})$ half $\mathrm{x}]$

toty $=[-f l i p l r($ halfy $)$ halfy $]$

epoxy $=1$ ine2(totx, toty)

else (for old way \#1)this strange sin*blah is so that at large angles the epoxy does not overlap itself and $\%$ therefore cause a problem.

$\%$ old way \#1 -- xtra $=$ halfx $(1,1)+\sin ($ psi_r $*$ pi/180 $) *(y \max +$ ant_thick $)$

$\%$ old way $\# 2--x \operatorname{xtra}=\max ($ raex $)+1 / 5 * \max$;

$\%$ xtra $=\min ($ raex $)+2 * x \max +$ ant_thick;

(halfx)-halfx (1);

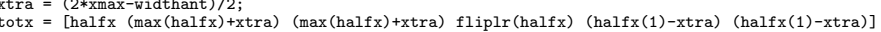

toty $=[$ halfy halfy(length(halfy)) -halfy(length(halfy)) -fliplr(halfy) -halfy(1) halfy(1)];

end

poxy $=$ line2 $($ tot $x$, toty)

els

"if psi $r=0$, we don't want to add extra epoxy or it will cause an error because the points

if will be too close to connect with a line

totx $=[\mathrm{fliplr}(\mathrm{halfx})$ halfx $]$

toty $=[-f l i p l r(h a l f y)$ halfy $]$

epoxy $=$ line 2 (tot $x$, toty)

else $\%$ (for old way \#1)this strange sin*blah is so that at large angles the epoxy does not overlap itself and

$\%$ therefore cause a problem.

old way \#1 -- xtra $=$ half $(1,1)+\sin ($ psi_r*pi/180 $) *(y \max +$ ant_thick $)$

$\%$ old way \#2-- xtra $=\max ($ raex $)+1 / 5 * x \max$;

$\%$ xtra $=\min ($ raex $)+2 *$ xmax+ant_thick;

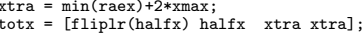

toty $=[$ liplr(halfy) -halfy - halfy $(1$, length (halfy $))$ halfy $(1$, length(halfy $))]$

end

epoxy $=$ line $2($ tot $x$, toty);

$\% \% \% \% \% \% \% \% \% \% \% \% \% \%$ end $\% \% \% \% \% \% \% \% \% \% \% \%$ end create inside antenna first $\% \% \% \% \% \% \% \% \% \% \% \% \% \% \% \% \% \% \% \% \% \% \% \% \% \% \%$

$\% \% \quad \% \% \% \% \% \% \%$ GOOD GRAPHING CODE

subplot $(2,2,1)$

scatter (xpoints, ypoints, 'r.'),

axis equal

hold scatter(nxpoints, nypoints, 'bx');

title(['shape', num2str(ndp), , , ,'curve number', num2str(k)])

pause

subplot $(2,2,2)$

plot (totx, toty ,' $r$ '), axis equal

subplot $(2,2,3)$

scatter(-raey,-raex, 'r.' ');

axis equal

scatter(raey,-raex, 'b.'),

plot (toty, -totx, 'r'

axis equal
title(['shape', num $2 \mathrm{str}$ (ndp),

$\% \% \% \% \% \% \% \% \% \% \% \% \% \% \% \% \% \% \% \% \%$

\% CALCULATE THE AREA FOR EACH ANTENNA
$\%$ step 1 -- create antenna elements

aer=line2 (raex, raey);

clear fem

fem.geom $=$ aer

fem.mesh $=$ meshinit $(f e m)$

\% Integrate on subdomain

$\mathrm{ae}_{\text {_vol }}=\operatorname{postint}\left(\mathrm{fem},{ }^{\prime}{ }^{\prime}\right)$;

\%\%\%\%\%\%\%\%\%\%\%\%BG DN 2D FEA\%\%\%\%\%\%\%\%\%\%\%\%\%\%\%\%\%\%\%\%\%\%\%\%\%\%\%\%\%\%\%\%

$\%$ calling file rather than inserting code

commandline_v9

\%\%\%\%\%\%\%\%\%\%\% 


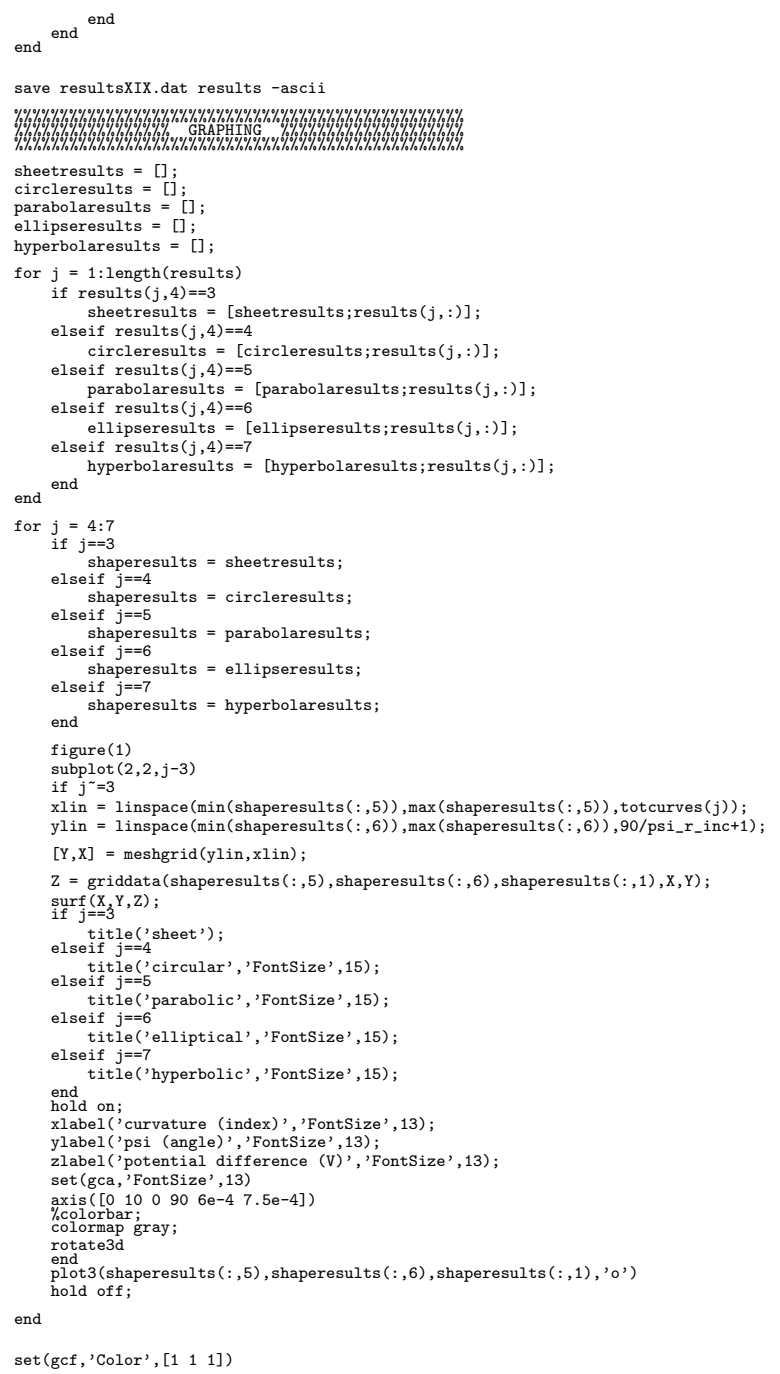




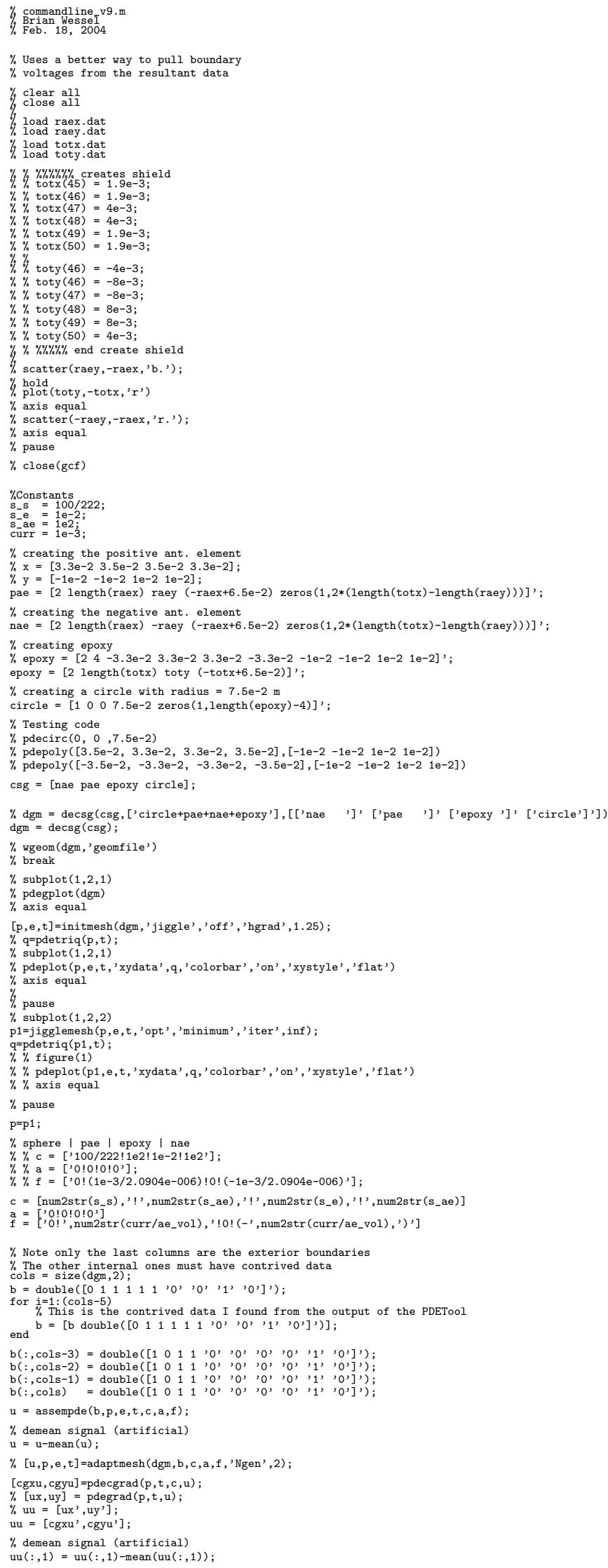


$\mathrm{uu}(:, 2)=\mathrm{uu}(:, 2)-\operatorname{mean}(\mathrm{uu}(:, 2))$;

$\% \%$ figure (2)

$\% \%$ pdeplot (p,e,t, 'xydata', u, 'mesh', 'off', 'contour', 'on' , 'levels', 30).

$\% \%$ set(gcf, 'Color', $\left.\left[\begin{array}{lll}1 & 1 & 1\end{array}\right]\right)$

$\% \%$ axis equal

$\% \%$ figure (3)

$\%$ title('Current Density')

$\% \%$ axis equal

$\%$ i=pdesde (e)

$\%$ finding boundary points and values

for $j=1$ : length $(p)$

$\operatorname{bpts}=\operatorname{norm}(p(:, j))$;

$\%$ display ('program paused')

if $(0.075-\mathrm{bpts})<1 \mathrm{e}-3$

$\% \quad$ (0.075-bpts)

$\% \quad$ display ('true')

boundv $(m)=u(j)$;

$\operatorname{boundp}(1, m)=p(1, j)$
boundp $(2, m)=p(2, j) ;$

$\% \quad$ display ('program paused - inside loop')

$x=p(1, j) ;$
$y=p(2, j) ;$

$\operatorname{theta}(m)=\operatorname{atan}(y / x)$

$\%$ fixing atan function so it goes from 0 to $2 * \mathrm{pi}$ rather than $-\mathrm{pi} / 2$ to $\mathrm{pi} / 2$ theta $(m)=\operatorname{theta}(m)+p i$

theta $(m)=\operatorname{theta}(m)+p i$
elseif $x<0$ \& $y<0$

theta $(m)=$ theta $(m)-p i$

end $m=m+1$

end

$\% \% \% \% \% \% \% \% \% \% \% \% \% \% \% \% \% \% \% \% \%$ GRAPHING

$\% \%$ subplot $(2,2,1)$

title('boundary voltage')

$\% \%$ subplot $(2,2,2)$

$\% \%$ polar (theta, boundv+1.1*min(boundv), 'r.')

$\% \%$ polar(theta, abs (boundv),'r.' ')

$\% \%$ subplot $(2,2,3)$

$\% \%$ stem3 (boundp $(1,:)$, boundp $(2,:)$, boundv , 'ro')

$\% \%$ title('boundary voltage')

$\% \%$ figure (3)

$\% \%$ polar (theta, abs (boundv), 'r.')

$\% \%$ title('boundary voltage')

$\% \% \% \% \% \% \% \% \% \% \% \% \% \% \% \% \% \% \% \%$

results $=[$ results; $(\max ($ boundv $)-\min ($ boundv $)) \max ($ boundv $) \min ($ boundv $)$ ndp k psi_r $]$;

clear p p1 q t u uu cgyu cgxu

\% display ('End of 2D FEA iteration - PROGRAM PAUSED')

$\%$ pause

$\%$ subplot $(2,2,3)$

$\%$ pdecont $(\mathrm{p}, \mathrm{t}, \mathrm{u}, 20)$ 


\section{.12 PDE TOOL CODE - NO EPOXY}

Contains the following files:

- create_antennas_noEpoxy.m

- commandline_v9.m (refer to .11)

- shapesfundersq.m (refer to .1)

- shapestotarclength.m (refer to .2)

- shapesptsopt_v2.m (refer to .2)

- normalpointsv2.m (refer to .3) 


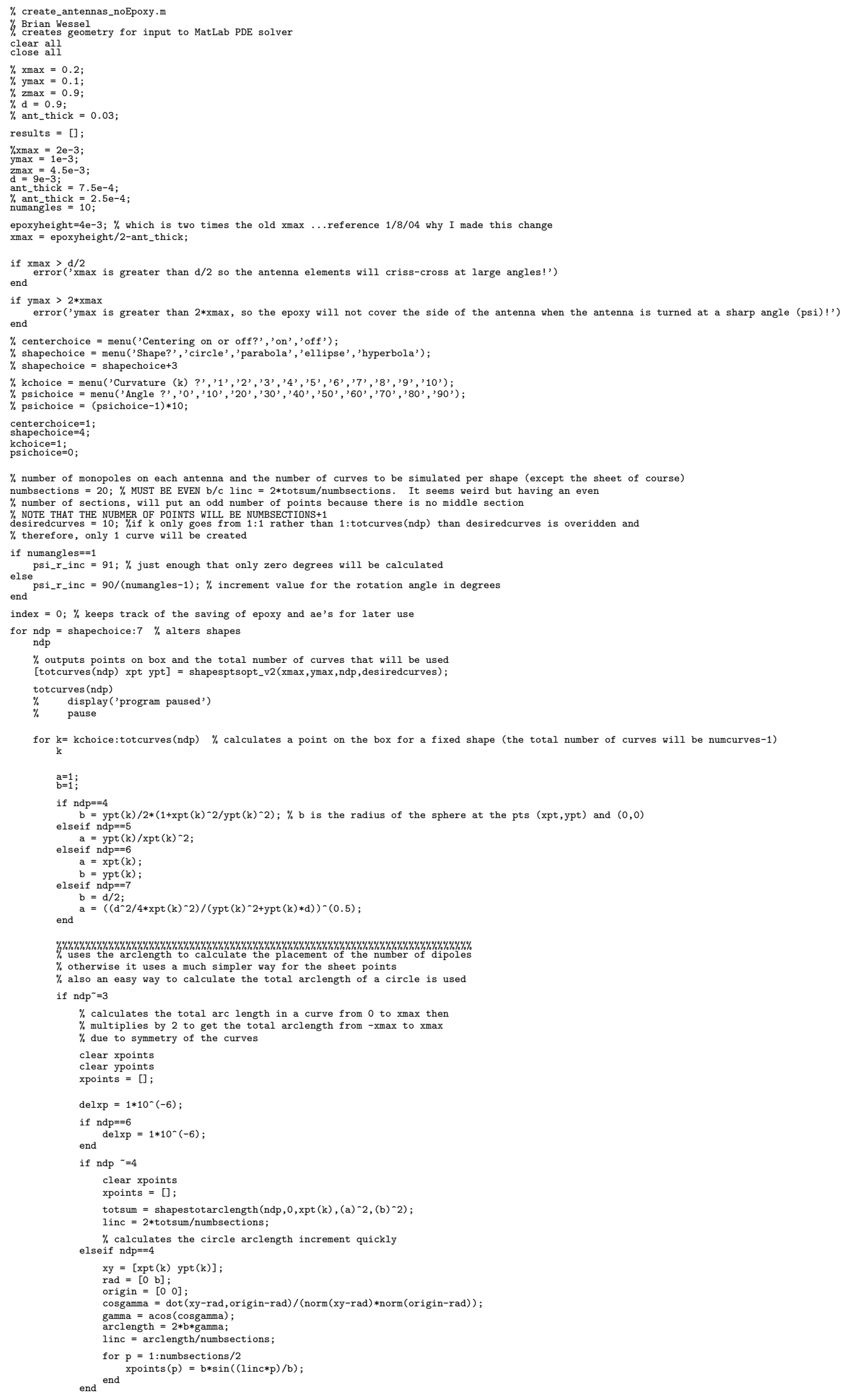




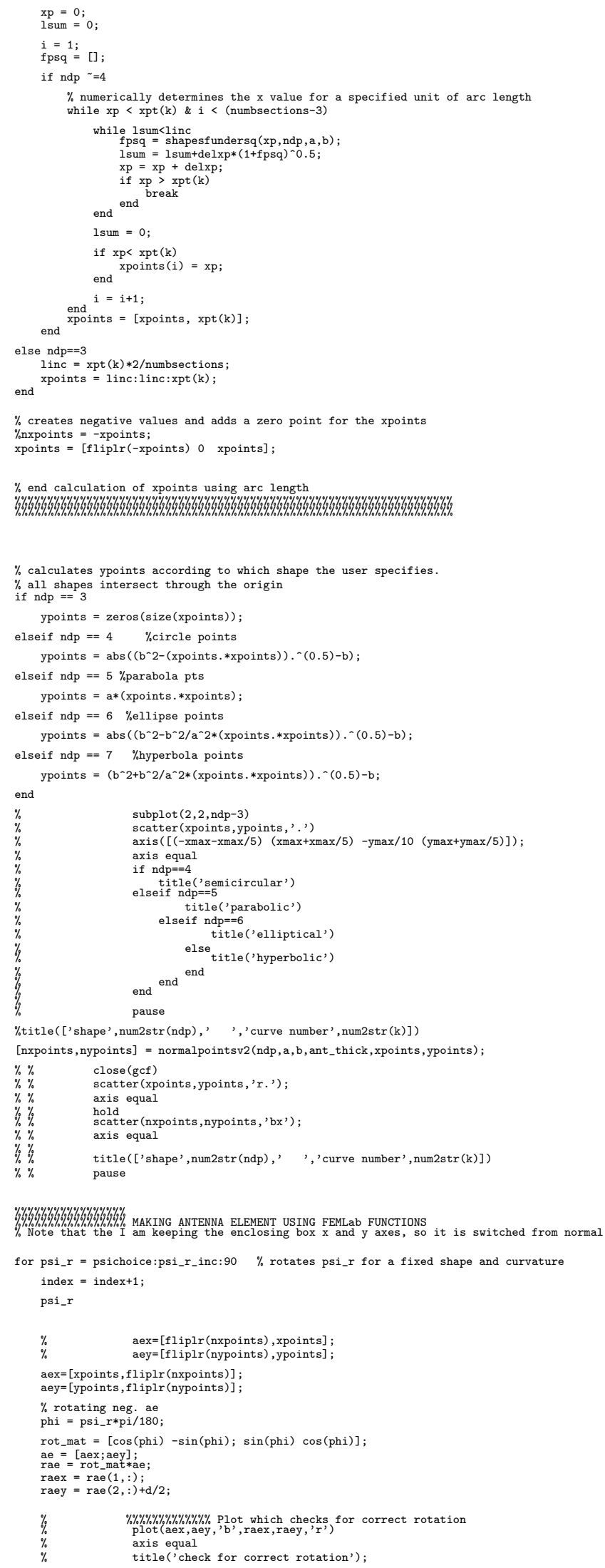




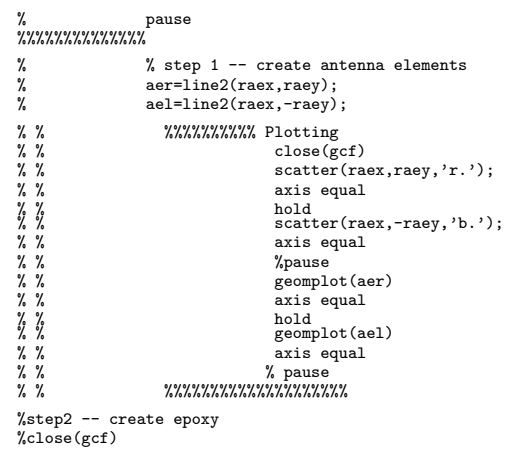

$\% \% \% \% \% \% \% \% \% \% \% \% \% \% \% \% \% \% \% \% \% \% \% \% \% \%$ code for creating outside antenna first. . uses output of normalptsv2.m $\% \% \% \% \% \% \% \% \% \% \% \% \% \% \% \% \% \% \% \% \% \% \% \% \% \% \% \%$ half $x=\operatorname{raex}($ numbsections $+2:$ length $($ raex $)$ )

halfy $=$ raey (numbsectionst $2:$ length (raey))

if centerchoice $=1 \%$ This choice will center the antenna element within the epoxy

$\%$ This regime is fundamentally different from noncentering because it should expose

$\%$ the difference between having a shield of epoxy vs free space and will give insigh

$\%$ into how much a shiend is effective at pushing the cree space and will give

$\%$ if psi_r $=0$, we don't want to add extra epoxy or it will cause an error because the points

$\%$ will be too close to connect with a line... however, I should add about half the antenna thickness

\% to bach sao clese the

if psi $x==0$ she

xtra $=$ ant_thick $/ 2$

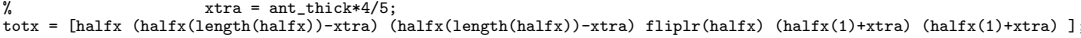
$\%$ toty $=[$ halfy halfy(length(halfy)) -halfy(length(halfy)) -fliplr(halfy) -halfy(1) halfy(1)]

\#1) this strange sintblat is so that

is strange sin*blah is so that at large angles the epoxy does not overlap itself and

.

$\%$ old way $\# 2--\mathrm{xtra}=\max ($ raex $)+1 / 5 * x \max$;

$\%$ xtra $=\min ($ raex $)+2 * x \max +$ ant_thick

half $=$ fliplr(half $x)$

halfy $=$ fliplr(halfy)

vidthant $=\max ($ half $\mathrm{x})$-half $\mathrm{x}(1)$

xout $=$ ant_thick*cos(phi)

xout $=$ ant_thick $* \cos ($ phi $) * 3 / 5 ;$
xtra $=($ epoxyheight-widthant $) / 2$

xtra $=($ epoxyheight-widthant $) * 4 / 5$;

$\% \quad$ yout $=$ ant_thick $* \sin (\mathrm{phi})$

ant_thick*sin (phi) *3/5;

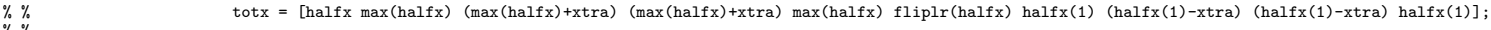

$\% \%$

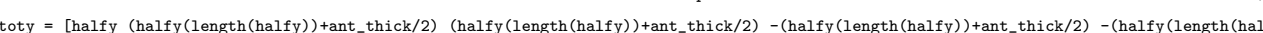

$\% \%$ Sophisticated Modeling which is memory intensive

totx $=$ halfx;
toty $=$ halfy;

$\% \quad \operatorname{tot} x=[\operatorname{totx}(\max (\operatorname{half} x)+x o u t)]$

totx $=[$ totx (halfx (length $($ half $x))+$ +xout) $)]$
toty $=[$ toty (halfy (length(halfy) + yout) $] ;$

totx $=[\operatorname{tot} x(\max ($ half $x)+x$ out $+x$ tra $)] ;$

totx $=[\operatorname{totx}(\max ($ half $x)+x$ out $+x$ tra $)] ;$
toty $=[\operatorname{toty}($ halfy $($ length $($ halfy $))+$ yout $)] ;$

totx $=[$ tot $(\max ($ half $\mathrm{x})+\mathrm{xout}+\mathrm{xtra})] ;$

toty $=[$ toty $-($ halfy $($ length $($ halfy $))+$ yout $)] ;$

$\% \quad \operatorname{tot} x=[\operatorname{totx}(\max ($ half $x)+x o u t)] ;$

tot $x=[$ totx (halfx (length $($ half $x))+$ xout $)] ;$
toty $=[$ toty $-($ halfy $($ length $($ halfy $))+$ yout $)]$

totx $=[\operatorname{totx}$ fliplr(halfx $)] ;$

toty $=[$ toty $-f l i p l r($ halfy $)]$

totx $=[$ totx (halfx (1)-xout) $)] ;$
toty $=[$ toty $-($ halfy $(1)-$ yout $)] ;$

totx $=[$ totx (halfx $(1)-x$ tra-xout $)] ;$
toty $=[$ toty $-($ halfy $(1)-$ yout $)] ;$

$\begin{aligned} \text { totx } & =[\text { totx }(\text { halfx }(1)-x \text { tra-xout })] \\ \text { toty } & =[\text { toty }(\text { halfy }(1)-\text { yout })] ;\end{aligned}$

totx $=[$ totx (halfx(1)-xout) $] ;$
toty $=[$ toty (halfy (1)-yout)
$\% \%$
$\% \%$
$\% \%$
$\% \%$
$\% \%$
$\% \%$
$\% \%$
$\% \%$
$\% \%$
$\% \%$
$\% \%$
$\% \%$
$\% \%$
$\% \%$
$\% \%$
$\% \%$
$\% \%$
$\% \%$
$\% \%$
$\% \%$
Primitive Modeling
totx $=[(\max ($ half $x)+x t r a)] ;$
toty $=[\min ($ halfy $)-$ ant_thick $/ 2]$
totx $=[$ totx $(\max ($ half $x)+x \operatorname{tra})] ;$
toty $=[$ toty $\max ($ halfy $)] ;$
totx $=[$ totx $(\max ($ half $x)+x$ tra + ant_thick $)] ;$
toty $=[$ toty $\max ($ halfy $)] ;$
$\begin{aligned} \text { totx } & =[\text { totx }(\max (\text { half } x)+x \text { tra+ant_thick })] ; \\ \text { toty } & =[\text { toty }-\max (\text { halfy })] ;\end{aligned}$
$\operatorname{totx}=[\operatorname{totx}(\max ($ half $x)+x \operatorname{tra})]$
toty $=[$ toty $-\max ($ halfy $y] ;$
totx $=[\operatorname{totx}(\max ($ halfx $)+x \operatorname{tra})] ;$
toty $=[\operatorname{toty}-(\min ($ halfy $)-$ ant_thick/2) $] ;$

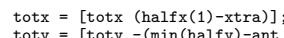

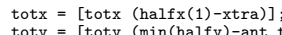


else

\%if psi_r $=0$, we don't want to add extra epoxy or it will cause an error because the points

\% will be too close to connect with a line

totx $=[$ fliplr(halfx) halfx $] ;$
toty $=[-$-fliplr(halfy) halfy $]$

else $\%$ (for old way \#1)this strange sin*blah is so that at large angles the epoxy does not overlap itself and

therefore cause a problem.

$\%$ old way \#1 -- xtra $=\operatorname{half} x(1,1)+\sin \left(p s i \_r * p i / 180\right) *\left(y \max +a n t \_t h i c k\right)$;

old way \#2 -- xtra $=\max ($ raex $)+1 / 5 * x \max$;

$\%$ xtra $=\min ($ raex $)+2 * x \max +$ ant_thick;

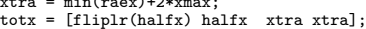
nd

toty $=[$ fliplr(halfy) - halfy - halfy $(1$, length (halfy $))$ halfy $(1$, length (halfy $))]$;

end $\% \% \% \% \% \% \% \% \% \% \% \% \% \% \% \% \% \% \% \% \% \%$ end create outside antenna first $\% \% \% \% \% \% \% \% \% \% \% \% \% \% \% \% \% \% \% \% \% \% \% \% \%$

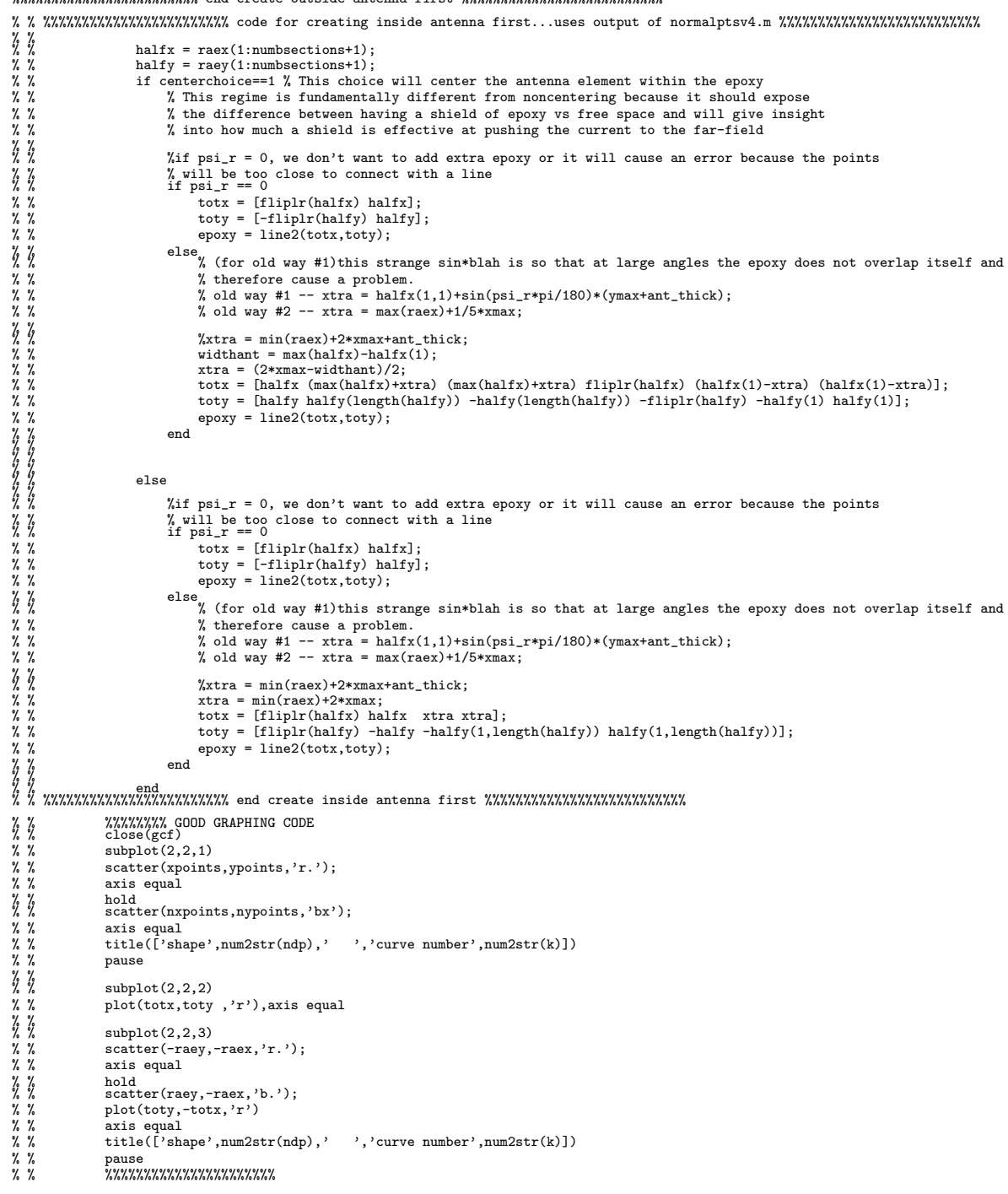

$\%$ CALCULATE THE AREA FOR EACH ANTENNA

aer=line2(raex, raey)

aer $=1$ ine2 2 (rem
\% Geometry

fem. geom $=$ aer

fem.mesh $=$ meshinit $(\mathrm{fem})$

ae_vol = postint (fem, ' 1 ');

\%\%\%\%\%\%\%\%\%\%\%\%,BEGIN 2D FEA\%\%\%\%\%\%\%\%\%\%\%\%\%\%\%\%\%\%\%\%\%\%\%\%\%\%\%\%\%\%\%\%\%

$\%$ calling file rather than inserting code

commandline_v9_noepoxy

\%\%\%\%\%\%\%\%\%\%\%\%,END 2D FEA \%\%\%\%\%\%\%\%\%\%\%\%\%\%\%\%\%\%\%\%\%\%\%\%\%\%\%\%\%\%\%\%\%\%\%\% 


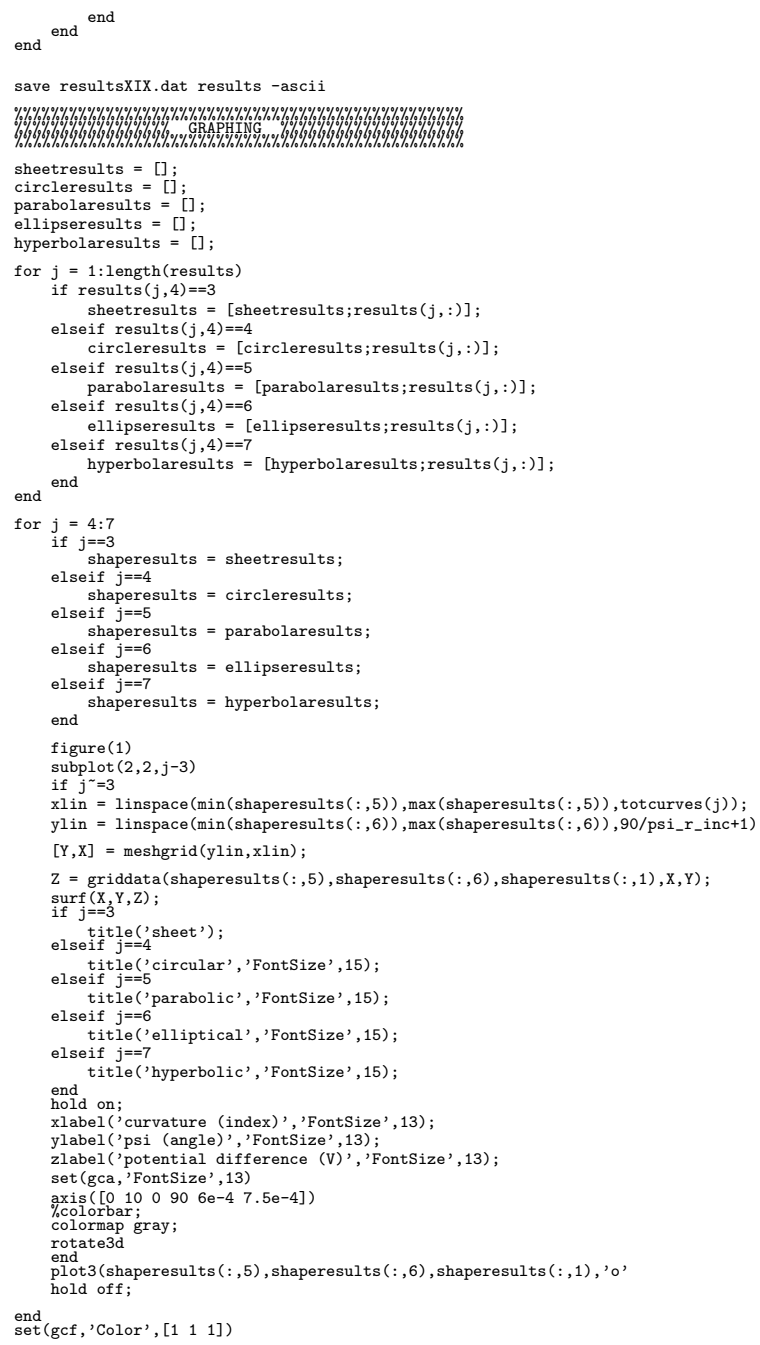

end
set(gcf, 'Color', [ $\left[\begin{array}{lll}1 & 1 & 1\end{array}\right]$ ) 


\section{.13 PDE TOOL CODE - REFLECTOR}

Contains the following files:

- create_antennas_hasReflector.m

- commandline_v9.m (refer to .11)

- shapesfundersq.m (refer to .1)

- shapestotarclength.m (refer to .2)

- shapesptsopt_v2.m (refer to .2)

- normalpointsv2.m (refer to .3) 
\% create-antennas_hasReflector.m

clear al1
close a11

$\% x \operatorname{xmax}=0.2$
$\%$ ymax $=0.1$

$\% \begin{aligned} & \text { ymax }=0.1 \\ & \text { zmax }=0.9\end{aligned}$

$\%$ d $=0.9 ;$
$\%$ ant_thick $=0.03$

results $=[]$;

$\% x \max =2 \mathrm{e}-3 ;$
$y \max =1 \mathrm{e}-3 ;$

$\mathrm{zmax}=4.5 \mathrm{e}-3$;

ant_thick $=7.5 \mathrm{e}-4 ;$
$\%$ ant_thick $=2.5 \mathrm{e}-4$

epoxyheight $=4 \mathrm{e}-3 ; \%$ which is two times the old $\mathrm{xmax}$...reference $1 / 8 / 04$ why I made this change

$\mathrm{xmax}=$ epoxyheight/2-ant_thick;

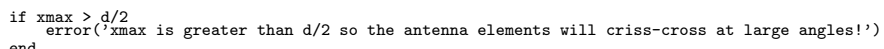

end

if $y \max >2 * x \max$

end

$\%$ centerchoice $=$ menu ('Centering on or off?' ', 'on' ' 'off' ');

$\%$ centerchoice $=$ menu('Centering on or off?', 'on', 'off');
$\%$ shapechoice $=$ menu('Shape?', 'circle', 'parabola', 'ellipse', 'hyperbola');
$\%$ shapechoice $=$ shapechoice+3;

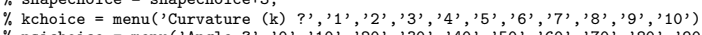

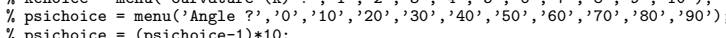

$\%$ psichoice $=($ psichoice-1) $* 10$

centerchoice $=1 ;$
shapechoice $=4 ;$

kchoice $=1 ;$
psichoice $=0$;

$\%$ number of monopoles on each antenna and the number of curves to be simulated per shape (except the sheet of course)

numbsections $=20 ; \%$ MUST BE EVEN $\mathrm{b} / \mathrm{c}$ linc $=2 *$ totsum/numbsections. It seems weird but having an even

$\%$ number of sections, will put an odd number of points because there is no middle section

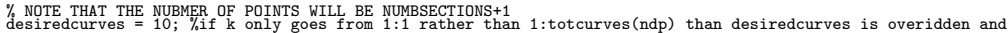

$\%$ therefore, only 1 curve will be created

psi_r_inc $=91 ; \%$ just enough that only zero degrees will be calculated

else psi_r_inc $=90 /$ (numangles-1); $\%$ increment value for the rotation angle in degrees

index $=0 ; \%$ keeps track of the saving of epoxy and ae's for later use

progress $=0$;
waitbar(progress/360,'2D FE Progress')

for ndp $=$ shapechoice: $7 \%$ alters shapes

$\%$ outputs points on box and the total number of curves that will be used

[totcurves (ndp) $\mathrm{xpt}$ ypt] = shapesptsopt_v2 (xmax, ymax, ndp, desiredcurves);

totcurves (ndp)

$\%$ display('program paused')

for $k=k$ choice:totcurves (ndp) \% calculates a point on the box for a fixed shape (the total number of curves will be numcurves-1)

$\mathrm{a}=1 ;$
$\mathrm{b}=1$

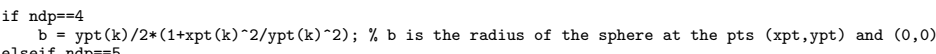

$a=y p t(k) / x p t(k) \wedge 2 ;$
elseif ndp==6

$\mathrm{a}=\operatorname{xpt}(\mathrm{k})$;

$\begin{aligned} a & =x p t(k) \\ b & =y p t(k) \\ b & \end{aligned}$

lseif ndp $==7$

$\mathrm{b}=\mathrm{d} / 2 ;$
$\mathrm{a}=((\mathrm{d} \sim 2 / 4 * \operatorname{xpt}(\mathrm{k}) \sim 2) /(\mathrm{ypt}(\mathrm{k}) \sim 2+\mathrm{ypt}(\mathrm{k}) * \mathrm{~d})) \sim(0.5)$

end

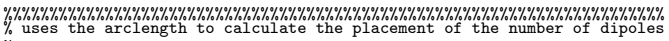

$\%$ otherwise it uses a much simpler way for the sheet points
$\%$ also an easy way to calculate the total arclength of a circle is used

if ndp $^{\sim}=3$

$\%$ calculates the total arc length in a curve from 0 to xmax then

$\%$ multiplies by 2 to get the total arclength from -xmax to xmax

$\%$ due to symmetry of the curves

clear xpoints

clear ypoints
xpoints $=[]$;

$\operatorname{delxp}=1 * 10^{\wedge}(-6) ;$

if $\mathrm{ndp}==6$

end

if ndp $\sim=4$

clear xpoints

totsum $=$ shapestotarclength $\left(\operatorname{ndp}, 0, \operatorname{xpt}(\mathrm{k}),(\mathrm{a})^{\sim} 2,(\mathrm{~b})^{\wedge}-2\right)$

linc $=2 *$ totsum/numbsections;

\% calculates the circle arclength increment quickly
elseif ndp $==4$

$x y=[x p t(k) y p t(k)]$

rad $=[0$ b];
origin $=\left[\begin{array}{ll}0 & 0\end{array}\right]$

cosgamma $=\operatorname{dot}(x y-r a d, o r i g i n-r a d) /($ norm $(x y-r a d) *$ norm $($ origin-rad $))$

gamma $=\operatorname{acos}(\cos g a m m a) ;$

arclength $=2 *$ b*gamma;

linc $=$ arclength/numbsections;

for $p=1:$ numbsections $/ 2$

$\operatorname{xpoints}(p)=b * \sin (($ linc $* p) / b)$, 


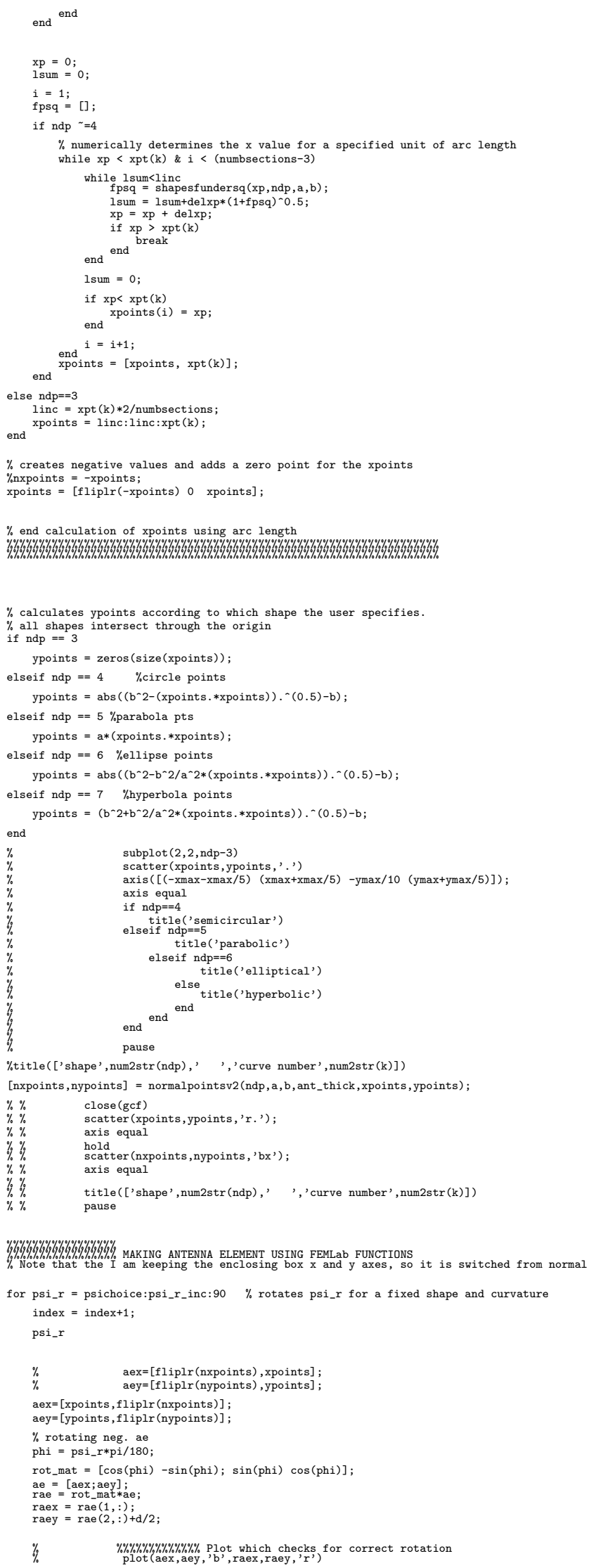

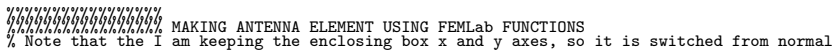

for psi_r $=$ psichoice:psi_r_inc:90 \% rotates psi_r for a fixed shape and curvature index $=$ index +1

psi_r

$\begin{array}{ll}\% & \text { aex }=[f l i p l r(\text { nxpoints), } \\ \% & \text { aey=[flipoints }] ;\end{array}$

aex $=[x p o i n t s$, fliplr (nxpoints) $]$;

$\%$ rotating neg. ae

rot_mat $=[\cos (\mathrm{phi})-\sin (\mathrm{phi}) ; \sin (\mathrm{phi}) \cos (\mathrm{phi})] ;$

ae $=[$ aex; $;$ aey $] ;$
rae $=$ rot mat $;$ mae;

raex $=\operatorname{rae}(1,:) ;$
raey $=$ rae $(2,:)+d / 2$

$\% \% \% \% \% \% \% \% \% \% \% \% \%$ plot which checks, for correct rotation
plot (aex, aey, 'b', raex, raey, 'r' $)$, 


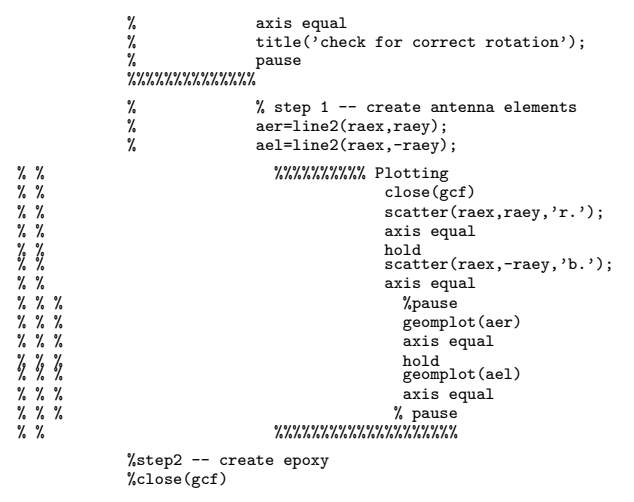

$\% \% \% \% \% \% \% \% \% \% \% \% \% \% \% \% \% \% \% \% \% \%$ code for creating outside antenna first . . uses output of normalptsv2.m \%\%\%\%\%\%\%\%\%\%\%\%\%\%\%\%\%\%\%\%\%\%\%\%\%

halfx $=$ raex (numbsections $+2:$ length $($ raex $)) ;$
halfy $=$ raey (numbsections $+2:$ length $($ raey));

halfy $=$ raey (numbsections +2 : length(raey));
if centerchoice $=1 \%$ This choice will center the antenna element within the epoxy

\% This regime is fundamentally different from noncentering because it should expose

$\%$ the difference between having a shield of epoxy vs free space and will give
$\%$ into how much a shield is effective at pushing the current to the far-field

$\%$ if psi_r $=0$, we don't want to add extra epoxy or it will cause an error because the points

$\%$ will be too close to connect with a line...however, I should add about half the antenna thickness

$\%$ elliptically shaped antenna elements

if $p s i \_r==0$

$\begin{aligned} \text { xtra } & =\text { ant_thick } / 2\end{aligned}$

$\% \quad$ xtra $=$ ant_thick $* 4 / 5$

totx $=[$ halfx (halfx(length(halfx))-xtra) (halfx(length(halfx))-xtra) fliplr(halfx) (halfx(1)+xtra) (halfx(1)+xtra) ]; toty $=[$ halfy halfy (length(halfy)) -halfy(length
epoxy $=$ line2(totx, toty);

els $\%$ (for old way \#1) this strange sin*blah is so that at large angles the epoxy does not overlap itself and

$\%$ therefore cause a problem

$\%$ old way \#1 -- xtra $=$ half $x(1,1)+\sin ($ psi_r $*$ pi $/ 180) *($ ymax+ant_thick $)$;

$\%$ old way \#2 -- xtra $=\max ($ raex $)+1 / 5 * \max$;

$\%$ xtra $=\min ($ raex $)+2 * x \max +a n t \_$thick;

half $=$ fliplr(half $x)$

halfy $=$ fliplr(halfy);
widthant $=\max ($ half

widthant $=\max ($ halfx $)$-halfx $(1)$;
xout $=$ ant_thick*cos $(\mathrm{phi})$

xout $=$ ant_thick $* \cos ($ phi $) * 3 / 5$

$\% \quad$ xtra $=($ epoxyheight-widthant $) / 2$

xtra $=$ (epoxyheight-widthant $) * 4 / 5$;

$\%$
$\%$ yout $=$ ant_thick $* \sin ($ phi $) * 3 / 5 ;$

$\%$ reflector thickness and width
rthick $=2 e-3$ rwidth $=1 \mathrm{e}^{-2}$

$\% \%$

$\% \%$

totx $=[$ half $\max ($ halfx $)(\max ($ halfx $)+x \operatorname{xta})(\max ($ halfx $)+x \operatorname{xta}) \max ($ halfx $)$ fliplr(halfx) halfx(1) (halfx(1)-xtra) (halfx(1)-xtra) halfx(1)];

$\% \%$ Sophisticated Modeling which is memory intensive

totx $=$ half $x ;$
toty $=$ halfy;

$\% \quad \operatorname{tot} x=[\operatorname{totx}(\max ($ half $\mathrm{x})+\mathrm{xout})] ;$

totx $=[$ totx (halfx $($ length $($ half $x))+$ xout $)] ;$
toty $=[$ toty (halfy (length(halfy)) + yout) $]$

totx $=[\operatorname{totx}(\max ($ half $x)+x$ out $)] ;$
toty $=[$ toty $($ halfy $($ length $($ halfy $))+$ rwidth $)] ;$

totx $=[$ totx $(\max ($ half $x)+$ xout + rthick $)] ;$
toty $=[$ toty $($ halfy $($ length $($ halfy $))+$ rwidth $)] ;$

totx $=[$ totx $(\max ($ half $x)+x$ out + rthick $)] ;$
toty $=[$ toty $-($ halfy $($ length $($ halfy $))+$ rwidth $)] ;$

$\% \quad \operatorname{tot} x=[\operatorname{totx}(\max ($ half $x)+x o u t)] ;$

totx $=[$ totx $(\max ($ half $\mathrm{x})+\mathrm{xout})] ;$
toty $=[$ toty $-($ halfy $($ length $($ halfy $))+$ rwidth $)]$

totx $=[$ totx $($ half $x($ length $($ half $x))+$ xout $)] ;$
toty $=[$ toty $-($ halfy $($ length (halfy $))+$ yout $)]$

$\begin{aligned} \text { totx } & =[\text { totx fliplr(half } x)] ; \\ \text { toty } & =[\text { toty - fliplr(half })]\end{aligned}$

totx $=[\operatorname{totx}(\operatorname{halfx}(1)-x$ out $)]$;

toty $=[$ toty $-($ halfy(1)-yout $)] ;$

totx $=[$ totx (halfx $(1)-x \operatorname{tra}-x$ out $)]$
toty $=[$ toty $-($ halfy $(1)-y o u t)] ;$

totx $=[\operatorname{totx}($ halfx $(1)-x$ tra-xout $)] ;$
toty $=[\operatorname{toty}($ halfy $(1)$-yout $)] ;$

totx $=[\operatorname{totx}($ half $x(1)-x o u t)] ;$

toty $=[\operatorname{toty}(\operatorname{halfy}(1)-$ yout $)]$

\begin{tabular}{|c|c|}
\hline \multirow{7}{*}{$\% \% \%$} & Primitive Modeling \\
\hline & $\begin{array}{l}\text { totx }=[(\max (\text { half } \mathrm{x})+\mathrm{xtra})] ; \\
\text { toty }=[\min (\text { halfy })-\text { ant_thick } / 2] ;\end{array}$ \\
\hline & $\begin{array}{l}\operatorname{totx}=[\operatorname{totx}(\max (\text { halfx })+\text { xtra })] ; \\
\text { toty }=[\text { toty } \max (\text { halfy })] ;\end{array}$ \\
\hline & $\begin{array}{l}\text { totx }=[\text { totx }(\max (\text { half } x)+\text { xtra+ant_thick })] ; \\
\text { toty }=[\text { toty } \max (\text { halfy })] ;\end{array}$ \\
\hline & $\begin{array}{l}\operatorname{tot} x=[\operatorname{totx}(\max (\text { half } x)+x \operatorname{xtra}+\text { ant_thick })] ; \\
\text { toty }=[\operatorname{toty}-\max (\text { halfy })] ;\end{array}$ \\
\hline & $\begin{array}{l}\operatorname{totx}=[\operatorname{totx}(\max (\text { half } x)+x \operatorname{xtra})] ; \\
\text { toty }=[\operatorname{toty}-\max (\text { halfy })] ;\end{array}$ \\
\hline & $\begin{array}{l}\operatorname{totx}=[\operatorname{totx}(\max (\text { half } x)+x t r a)] ; \\
\text { toty }=[\text { toty }-(\min (\text { halfy })-\text { ant_thick } / 2)] ;\end{array}$ \\
\hline
\end{tabular}




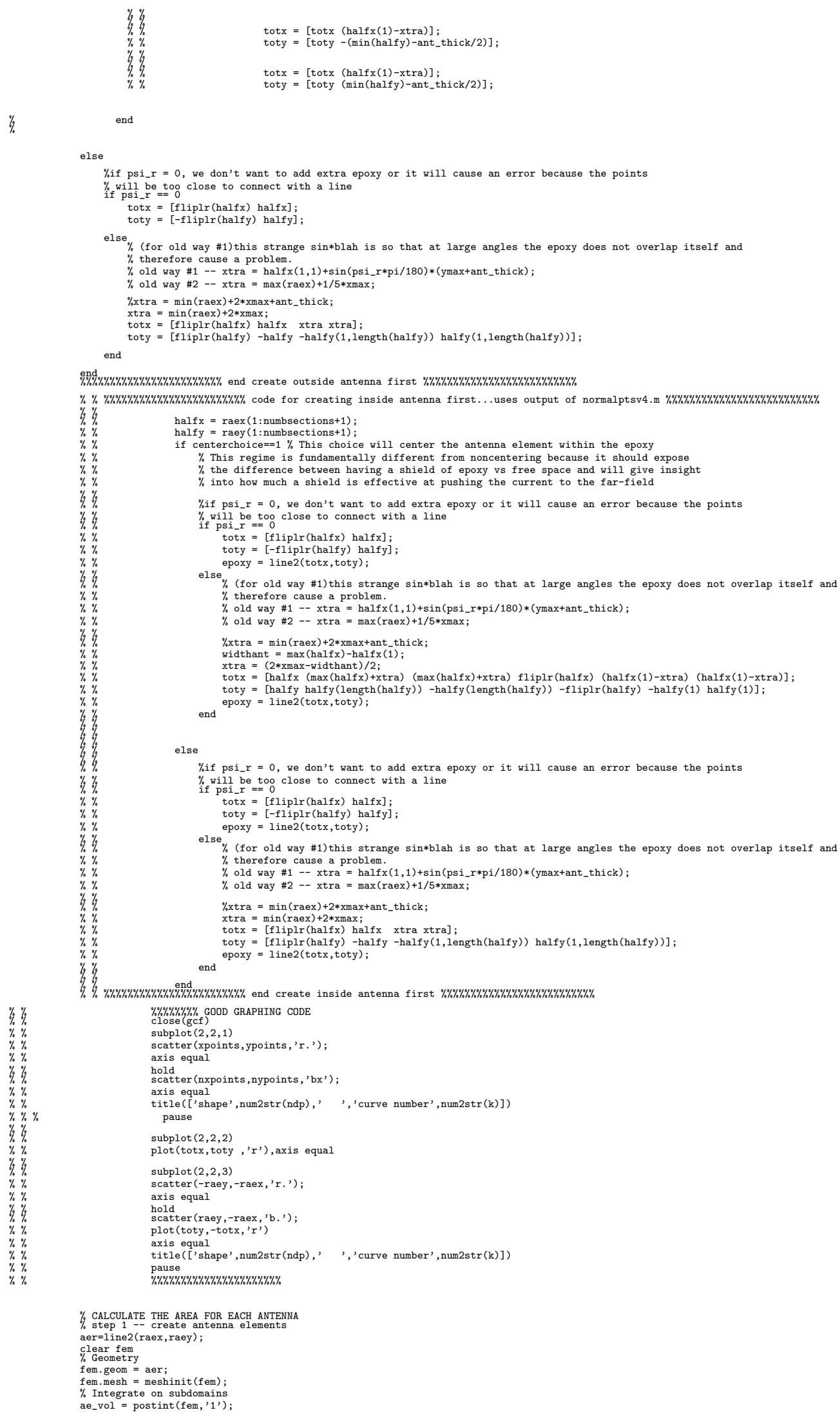

end $\% \% \% \% \% \% \% \% \% \% \% \% \% \% \% \% \% \% \% \% \%$ end create outside antenna first \%\%\%\%\%\%\%\%\%\%\%\%\%\%\%\%\%\%\%\%\%\%\%\%\%

$\% \% \% \% \% \% \% \% \% \% \% \% \% \% \% \% \% \% \% \% \% \% \% \% \%$ code for creating inside antenna first. . uses output of normalptsv 4 m $\% \% \% \% \% \% \% \% \% \% \% \% \% \% \% \% \% \% \% \% \% \% \% \% \% \%$

$\% \% \quad$ halfx $=\operatorname{raex}(1:$ numbsections +1$) ;$

halfx $=$ raex $(1:$ numbsections +1$) ;$
halfy $=$ raey $(1:$ numbsections +1$)$

$\% \%$ if centerchoice==1 \% This choice will center the antenna element within the epoxy

\% This regime is fundamentally different from noncentering because it should expose

$\%$ the difference between having a shield of epoxy vs free space and will give insight

$\%$ into bou mach a shield is effective at pe epoxy vs free spece and will give ir

\%if psi_r $=0$, we don't want to add extra epoxy or it will cause an error because the points

$\%$ will be too close to connect with a line

totx $=[\mathrm{flip} \ln ($ half $\mathrm{x})$ half $\mathrm{x} ;$

toty $=[-f l i p l r(h a l f y)$ half $y]$

else (for old way \#1)this strange sin*blah is so that at large angles the epoxy does not overlap itself and $\%$ therefore cause a problem. $\%$ old way \#1-- xtra $=$ half $(1,1)+\sin ($ psi_r*pi/180)* (ymax+ant_thick); (raex) $+1 / 5 * \max$

$\%$ xtra $=\min ($ raex $)+2 * x \max +a n t \_$thick;

widthant $=\max ($ half $\mathrm{x})$-halfx $(1)$;

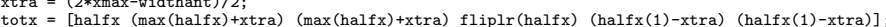
toty $=[$ halfy halfy(length(halfy)) -halfy(length(halfy)) -fliplr(halfy) -halfy(1) halfy(1)];

$\% \% \% \% \% \% \% \% \% \% \%$ end $1 \% \% \% \% \% \% \% \% \% \% \%$ end create inside antenna first $\% \% \% \% \% \% \% \% \% \% \% \% \% \% \% \% \% \% \% \% \% \% \% \% \% \%$

$\% \% \% \% \% \%$ GOOD GRAPHING CODE
close (gcf)

subplot $(2,2,1)$

scatter(xpoints, ypoints, 'r.')

axis equal

hcatter (nxpoints, nypoints, 'bx');

axis equal
title(['shape', num2str(ndp), , , ' 'curve number', num2str(k)])

pause

subplot $(2,2,2)$

plot (totx, toty ,' $r$ '), axis equal

subplot $(2,2,3)$

scatter(-raey, -raex, 'r.');

axis equal

scatter (raey, -raex, 'b.' ');

plot (toty, -totx, ' $r$ ')

title(['shape', num2str(ndp),' ', 'curve number', num2str(k)])

$\% \% \% \% \% \% \% \% \% \% \% \% \% \% \% \% \% \% \% \% \% \%$

\% CALCULATE THE AREA FOR EACH ANTENNA

aer $=1$ ine2 (raex, raey);

\% Gear fem

fem.geom = aer;

fem.mesh = meshinit (fem);

$\%$ Integrate on subdomain

ae_vol = postint $\left(f_{e m},{ }^{\prime}{ }^{\prime}\right)$; 


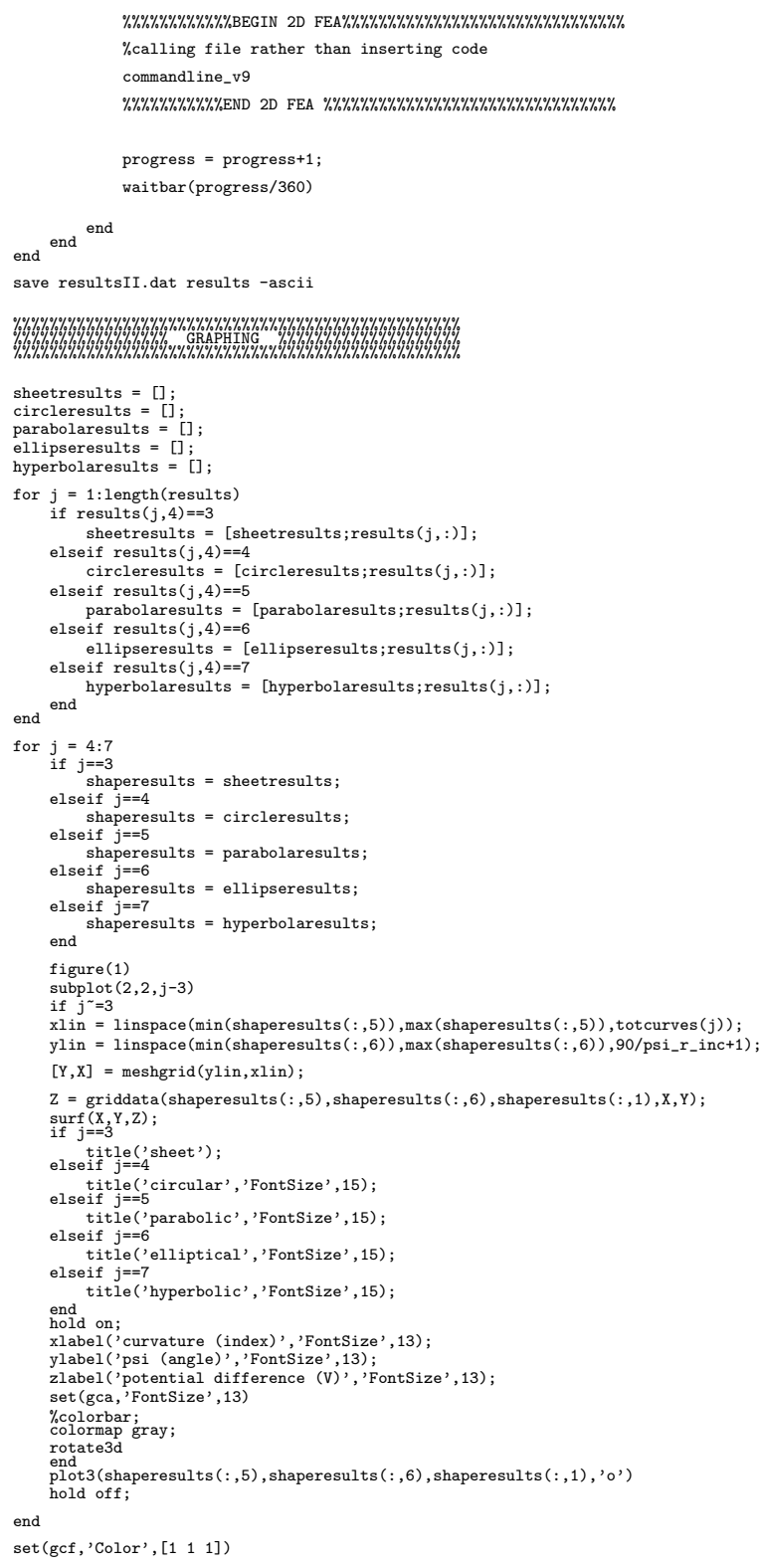




\section{.14 PDE MULTISHELL (WITH EPOXY)}

Contains the following files:

- create_antennas_noReflector.m

- commandline_v9.m (refer to .11)

- shapesfundersq.m (refer to .1)

- shapestotarclength.m (refer to .2)

- shapesptsopt_v2.m (refer to .2)

- normalpointsv2.m (refer to .3) 
\% Brian Wessel creates geometry for input to MatLab PDE solver

clear al1
close al1

$\% x \max =0.2 ;$

$\%$ ymax $=0.1$

$\%, z \max =0.9 ;$

$\%$ ant_thick $=0.03$;

results $=[] ;$

progress $=0$;

\% $x \max =2 \mathrm{e}-3 ;$
$y \max =1 \mathrm{e}-3 ;$

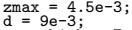

ant_thick $=7.5 \mathrm{e}-4$;

$\%$ ant thick $=2.5 e^{2}-4 ;$
numangles $=10 ;$

epoxyheight $=4 e-3 ; \%$ which is two times the old xmax ...reference $1 / 8 / 04$ why I made this change

$x \max =$ epoxyheight $/ 2$-ant_thick;

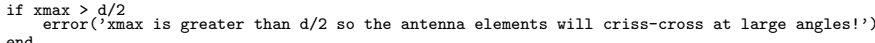

if $y \max >2 * x \max$

end error ('ymax is greater than $2 * x \max$, so the epoxy will not cover the side of the antenna when the antenna is turned at a sharp angle (psi)!')

$\%$ centerchoice $=$ menu ('Centering on or off?', 'on' , 'off');

$\%$ shapechoice = menu ('Shape?', 'circle', 'parabola', 'ellipse', 'hyperbola')

$\%$ shapechoice $=$ shapechoice+3;

$\%$ kchoice = menu('Curvature (k) ?', '1, ', '2, '3', '4, ',5', '6, ',7', '8, ', ', '10');

\% psichoice $=$ menu (

centerchoice $=1$;
shapechoice $=4 ;$

\section{kchoice $=1 ;$
psichoice $=0$;}

$\%$ number of monopoles on each antenna and the number of curves to be simulated per shape (except the sheet of course)

numbsections $=20 ; \%$ MUST BE EVEN $\mathrm{b} / \mathrm{c}$ linc $=2 *$ totsum/numbsections. It seems weird but having an even

\% NOTE THAT THE NUBMER OF POINTS WILL BE NUMBSECTIONS+1 $1:$ totcurves(ndp) than desiredcurves is overidden and

$\%$ therefore, only 1 curve will be created

if numangles $==1$

psi_r_inc $=91 ; \%$ just enough that only zero degrees will be calculated

else psi_r_inc $_{\text {in }}=90 /($ numangles-1); $\%$ increment value for the rotation angle in degrees

index $=0 ; \%$ keeps track of the saving of epoxy and ae's for later use

for ndp $=$ shapechoice: $7 \%$ alters shapes

$\%$ outputs points on box and the total number of curves that will be used
[totcurves (ndp) xpt ypt] = shapesptsopt_v2(xmax ymax, ndp, desiredcurves);

totcurves (ndp)

$\% \quad$ display ('program paused')

for $\mathrm{k}=\mathrm{kchoice}:$ totcurves(ndp) \% calculates a point on the box for a fixed shape (the total number of curves will be numcurves-1)

$\mathrm{a}=1 ;$
$\mathrm{b}=1 ;$

if $\mathrm{ndp}=4$

if ndp==4
$b=y p t(k) / 2 *(1+x p t(k)-2 / y p t(k)-2) ; \% b$ is the radius of the sphere at the pts $(x p t, y p t)$ and $(0,0)$
elseif ndp $==5$

$a=y p t(k) / x p t(k)-2$

seif ndp $==6$

$a=\operatorname{xpt}(k)$
$b=\operatorname{ypt}(k)$

$\begin{aligned} & \mathrm{b}=\mathrm{ypt}(\mathrm{k}) \\ & \text { elseif ndp }==7\end{aligned}$

$\mathrm{b}=\mathrm{d} / 2$

end

$=\left(\left(d^{`} 2 / 4 * \operatorname{xpt}(k)-2\right) /(\operatorname{ypt}(k) \sim 2+y p t(k) * d)\right)-(0.5)$

$\% \% \% \% \% \% \% \% \% \% \% \% \% \% \% \% \% \% \% \% \% \% \% \% \% \% \% \% \% \% \% \% \% \% \% \% \% \% \% \% \% \% \% \% \% \% \% \% \% \% \% \% \% \% \% \% \% \% \% \% \% \% \% \% \% \% \% \% \% \% \% \% \% \% \% \% \% \% \% \% \% \% \% \% \% \% \% \% \% \%$

$\%$ otherwise it uses a much simpler way for the sheet points

$\%$ also an easy way to calculate the total arclength of a circle is used

if $\mathrm{ndp}^{\sim}=3$

$\%$ calculates the total arc length in a curve from 0 to $x \max$ then

$\%$ multiplies by 2 to get the total arclength from -xmax to $x \max$

clear xpoint

clear ypoints
xpoints $=[]$;

$\operatorname{delxp}=1 * 10^{\wedge}(-6)$

if $\mathrm{ndp}==6$

delxp $=1 * 10^{\wedge}(-6)$

if ndp $\sim=4$

clear xpoints
xpoints $=[] ;$

totsum $=$ shapestotarclength (ndp, $0, \operatorname{xpt}(\mathrm{k}),(\mathrm{a}) \wedge 2,(\mathrm{~b}) \wedge 2)$

linc $=2 *$ totsum/numbsections;

$\%$ calculates
elseif ndp $==4$

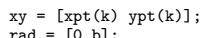

$\mathrm{rad}=\left[\begin{array}{ll}0 & \mathrm{~b}\end{array}\right]$
origin $=[0]$

cosgamma $=\operatorname{dot}(x y-r a d$, origin-rad $) /($ norm $(x y-r a d) *$ norm (origin-rad $))$

gamma $=\operatorname{acos}($ cosgamma)

arclength $=2 * \mathrm{~b} *$ gamma

linc $=$ arclength/numbsections

for $p=1:$ numbsections $/ 2$

end ${ }^{\text {end }}$

$\operatorname{xpoints}(\mathrm{p})=\mathrm{b} * \sin ((\operatorname{lin} \mathrm{c} * \mathrm{p}) / \mathrm{b})$; 


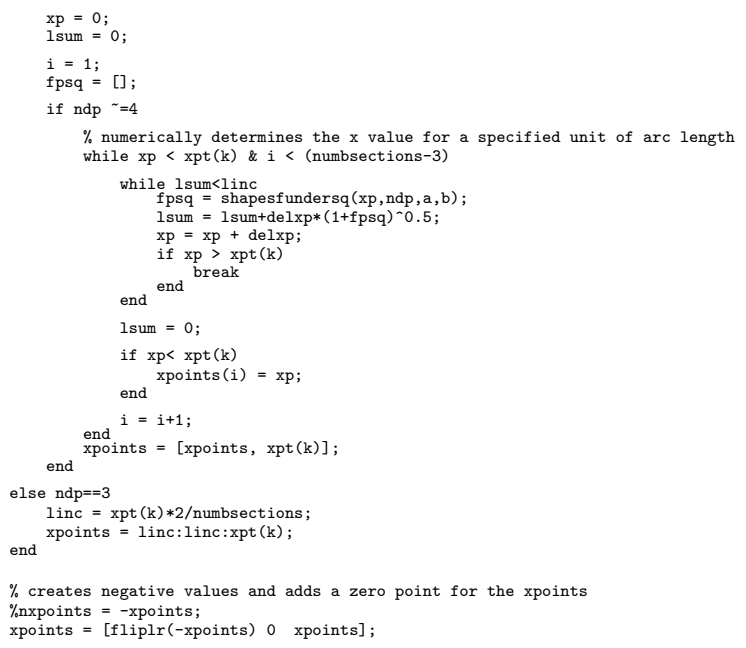

$\% \% \% \% \% \% \% \% \% \% \% \% \% \% \% \% \% \% \% \%$ MAKING ANTENNA ELEMENT USING FEMLab FunCTIONS

for psi_r $=$ psichoice:psi_r_inc: $90 \%$ rotates psi_r for a fixed shape and curvature index $=$ index +1

psi_r

$\% \quad$ aex=[fliplr(nxpoints), xpoints];

aex $=[$ xpoints, fliplr (nxpoints) $]$;

aey=[ypoints, fliplr(nypoints)];

$\%$ rotating neg. ae
phi $=$ psi_r*pi $/ 180 ;$

rot_mat $=[\cos (\mathrm{phi})-\sin (\mathrm{ph} \mathrm{i}) ; \sin (\mathrm{phi}) \cos (\mathrm{phi})]$

ae $=[$ aex; aey $] ;$
rae $=$ rot_mat $*$ ae

raex $=\operatorname{rae}(1,:) ;$
raey $=\operatorname{rae}(2,:)+d / 2$

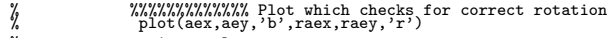

$\% \quad$ title('check for correct rotation'); 
else

\%if psi_r $=0$, we don't want to add extra epoxy or it will cause an error because the points

\% will be too close to connect with a line

totx $=[$ fliplr(halfx) halfx $] ;$
toty $=[-$-fliplr(halfy) halfy $]$

else $\%$ (for old way \#1)this strange sin*blah is so that at large angles the epoxy does not overlap itself and

therefore cause a problem.

old way \#1 -- xtra = half $x(1,1)+\sin \left(p s i \_r * p i / 180\right) *($ ymax+ant_thick);

old way \#2 -- xtra $=\max ($ raex $)+1 / 5 * x \max$;

$\%$ xtra $=\min ($ raex $)+2 * x \max +$ ant_thick;

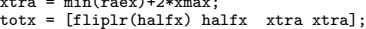
nd

toty $=[$ fliplr(halfy) - halfy - halfy $(1$, length (halfy $))$ halfy $(1$, length (halfy $))]$;

end $\% \% \% \% \% \% \% \% \% \% \% \% \% \% \% \% \% \% \% \% \% \%$ end create outside antenna first $\% \% \% \% \% \% \% \% \% \% \% \% \% \% \% \% \% \% \% \% \% \% \% \% \%$

$\% \% \% \% \% \% \% \% \% \% \% \% \% \% \% \% \% \% \% \% \% \% \% \% \% \% \%$ code for creating inside antenna first . . uses output of normalptsv 4 m $\% \% \% \% \% \% \% \% \% \% \% \% \% \% \% \% \% \% \% \% \% \% \% \% \% \% \% \% \%$ $\begin{array}{ll}\% \% & \text { half }=\text { raex }(1 \text { :numbsections }+1) ; \\ \% \% & \text { halfy }=\operatorname{raey}(1: \text { numbsections }+1) ;\end{array}$

if centerchoice $=1 \%$ This choice will center the antenna element within the epoxy

$\%$ This regime is fundamentally different from noncentering because it should expose

$\%$ the difference between having a shield of epoxy vs free space and will give

\%if psi_r $=0$, we don't want to add extra epoxy or it will cause an error because the points

$\%$ will be too close to connect with a line

totx $=[$ fliplr(halfx) halfx $] ;$

toty $=[-f l i p l r($ halfy) halfy $]$

epoxy $=\operatorname{line} 2($ tot $x$, toty)

else $\%$ (for old way \#1)this strange sin*blah is so that at large angles the epoxy does not overlap itself and $\%$ therefore cause a problem.

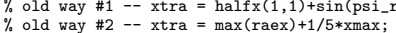

$\%$ xtra $=\min ($ raex $)+2 *$ xmaxtant_thick;

widthant $=\max ($ half $\mathrm{x})$-half $\mathrm{x}(\mathrm{1})$;

xtra $=(2 * x \max$-widthant $) / 2 ;$
totx $=[$ half $(\max ($ half $)+x \operatorname{tra})(\max ($ half $x)+x \operatorname{tra})$ fliplr(halfx) (halfx(1)-xtra) (halfx(1)-xtra) $]$

end oty $=[$ halfy halfy(length(halfy)) -halfy(length(halfy)) -fliplr(halfy) -halfy(1) halfy(1)];

else

\%if psi_r $=0$, we don't want to add extra epoxy or it will cause an error because the point

$\%$ will be too close to connect with a line
if $p s i$ i $==0$

totx $=[f \operatorname{lip} \operatorname{lr}($ half $x)$ half $x ;$

toty $=[-f l i p l r(h a l f y)$ halfy $]$

else epoxy $=1^{2}$

$\%$ (for old way \#1)this strange sin*blah is so that at large angles the epoxy does not overlap itself and $\%$ therefore cause a problem.

\% old way \#1 -- xtra $=$ halfx $(1,1)+\sin ($ psi_r*pi/180 $) *(y \max +$ ant_thick $)$

ex) $+1 / 5 * x \max$

$\%$ xtra $=\min ($ raex $)+2 * x \max +$ ant_thick;

$x \operatorname{tra}=\min ($ raex $)+2 * x \max$

totx $=$ [flplr(half ) halfx xtra $x$ tra]

-halfy -halfy(1, length(halfy)) halfy(1, length(halfy))]

end

$\% \% \% \% \% \% \% \% \% \% \% \% \% \% \% \% \% \% \% \% \% \% \%$ end create inside antenna first $\% \% \% \% \% \% \% \% \% \% \% \% \% \% \% \% \% \% \% \% \% \%$

$\% \% \quad \begin{aligned} & \% \% \% \% \% \% \% \text { GOOD GRAPHING CODE } \\ & \text { close (gcf) }\end{aligned}$

subplot $(2,2,1)$

scatter (xpoints, ypoints, 'r. ')

axis equal

hold scatter(nxpoints, nypoints, 'bx'),

axis equal

title(['shape', num2str(ndp),' , , 'curve number', num2str(k)])

pause

subplot $(2,2,2)$

plot (totx, toty , 'r'), axis equal

subplot $(2,2,3)$

scatter(-raey,-raex, 'r.');

axis equal

hold 2 (raey, -raex, 'b, ')

plot (toty,-totx, 'r')

axis equal

title(['shape', num2str(ndp),' ', 'curve number', num2str(k)])

pouse $\% \% \% \% \% \% \% \% \% \% \% \% \% \% \% \% \%$

$\%$ step 1 -- \% Creatculate antenna the AREA FOR EACH ANTENNA

aer=line2 (raex, raey);

\% Gear fem

fem.geom $=$ aer;

fem.mesh $=$ meshinit $(\mathrm{fem})$;

ae_vol = postint $($ fem, ' 1 ');

\%\%\%\%\%\%\%\%\%\%\%,BEG DN 2D FEA\%\%\%\%\%\%\%\%\%\%\%\%\%\%\%\%\%\%\%\%\%\%\%\%\%\%\%\%\%\%

$\%$ calling file rather than inserting code

commandline_v9_Multishell

\%\%\%\%\%\%\%\%\%\%\%END 2D FEA \%\%\%\%\%\%\%\%\%\%\%\%\%\%\%\%\%\%\%\%\%\%\%\%\%\%\%\%\%\%\%\%\%

progress $=$ progress +1 
waitbar(progress/360)

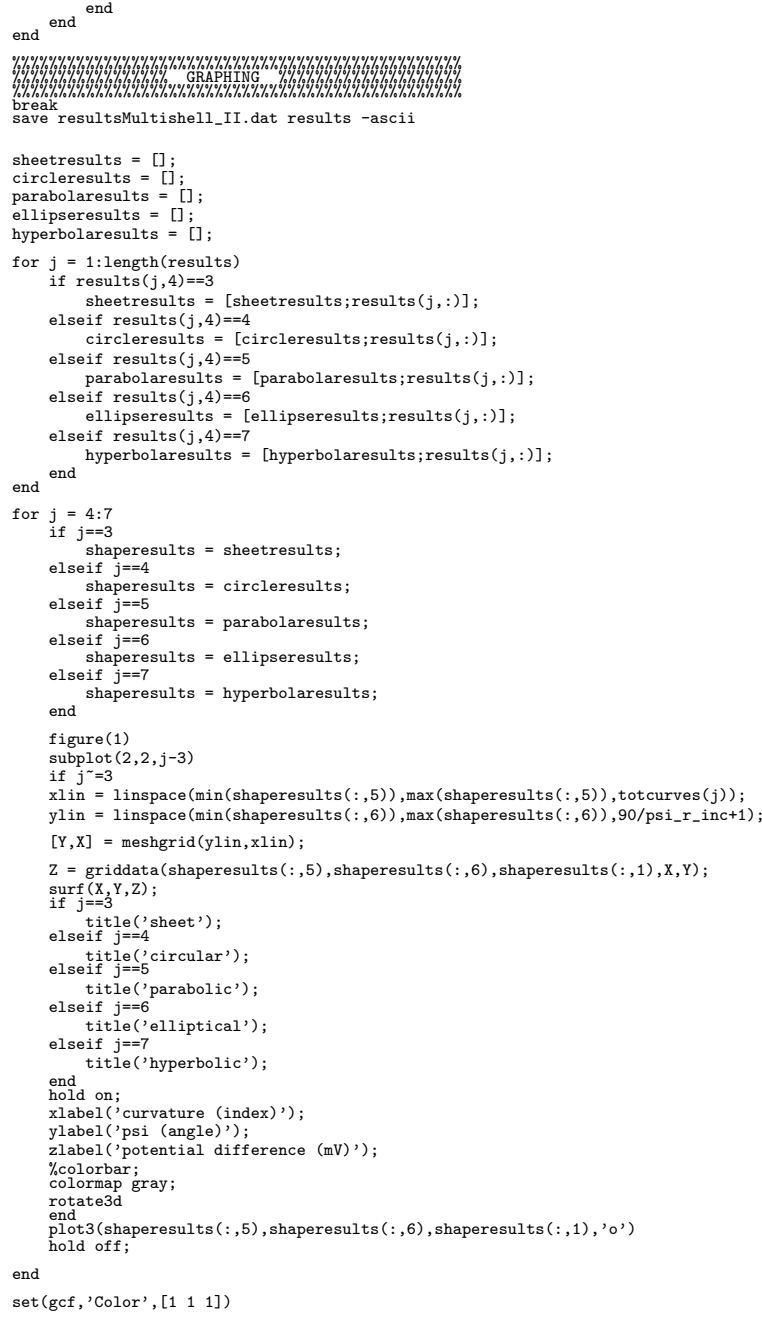




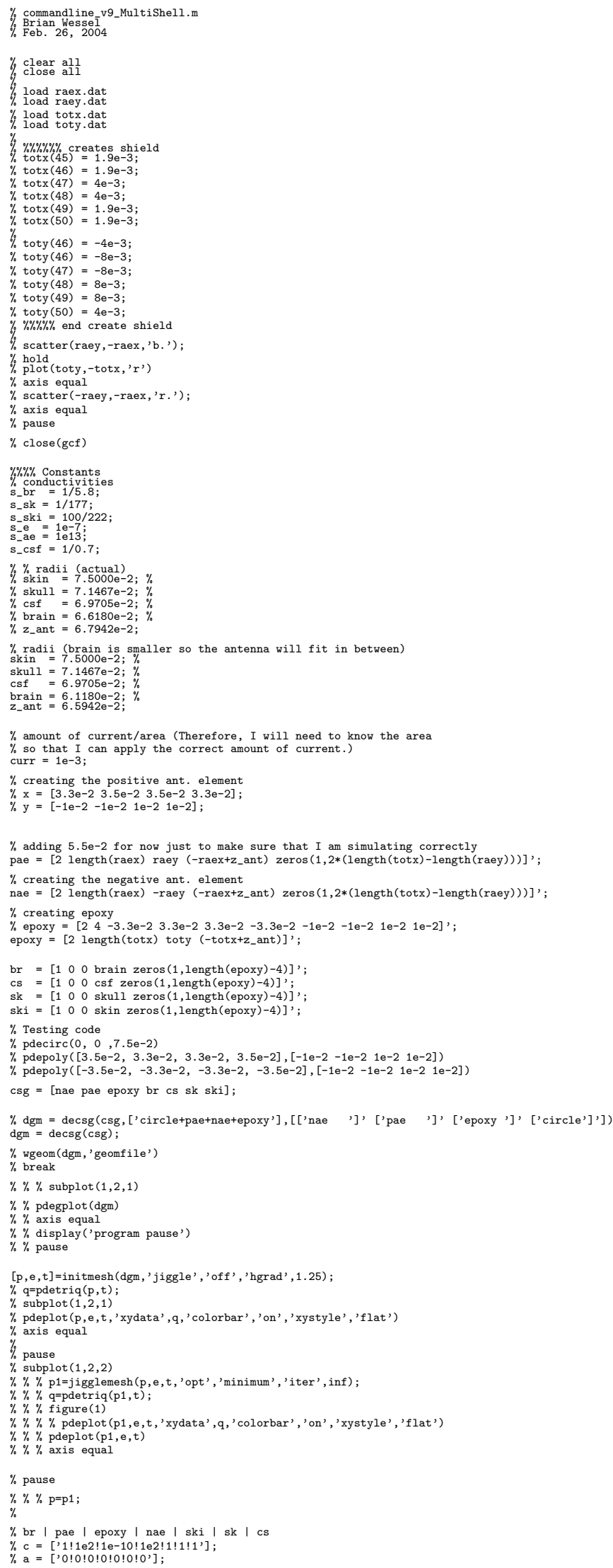




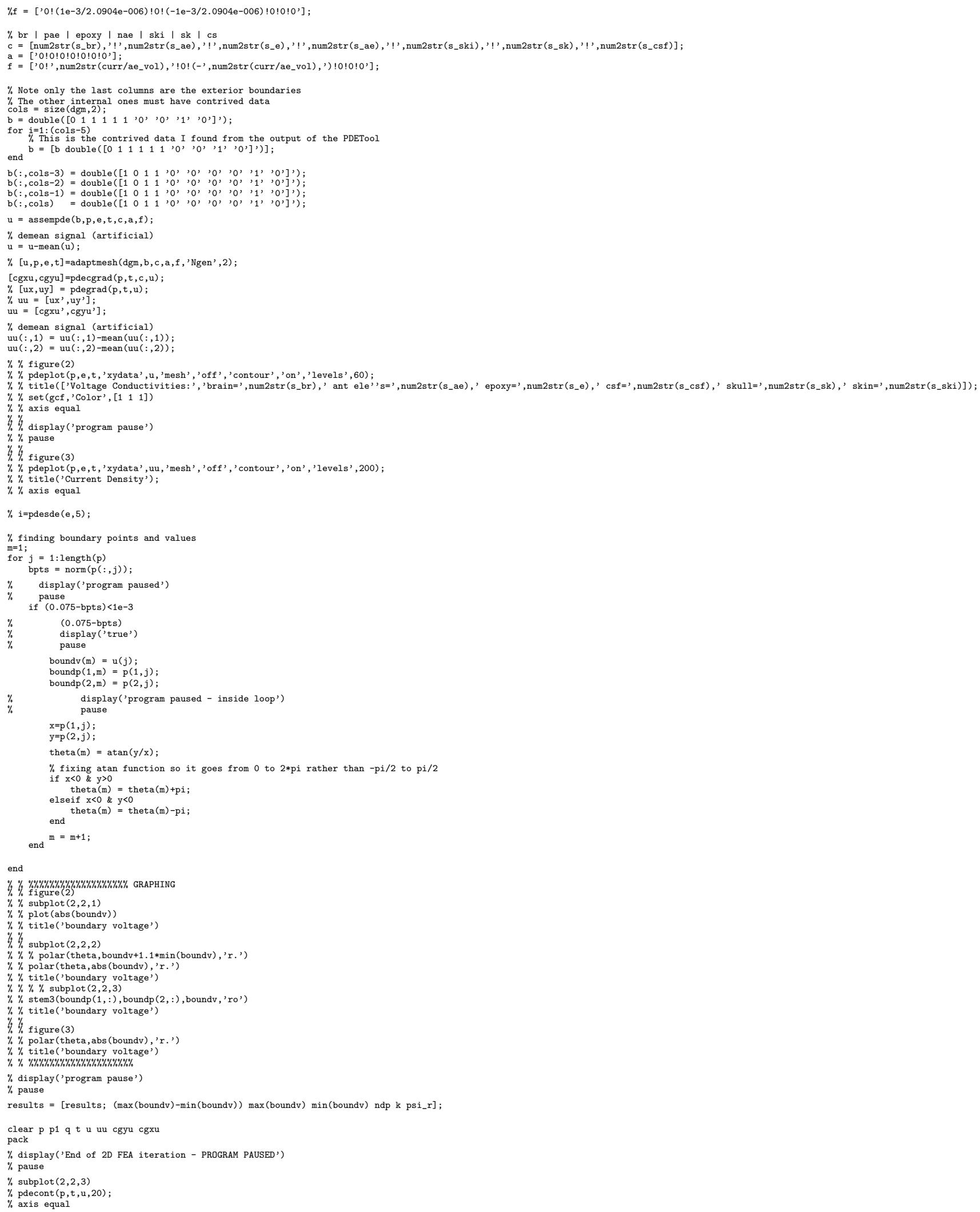




\section{.15 PLOTTING - EPOXY WITH \% INCREASE FROM ANALYTICAL}

Contains the following file:

- EpoxyResults.m 


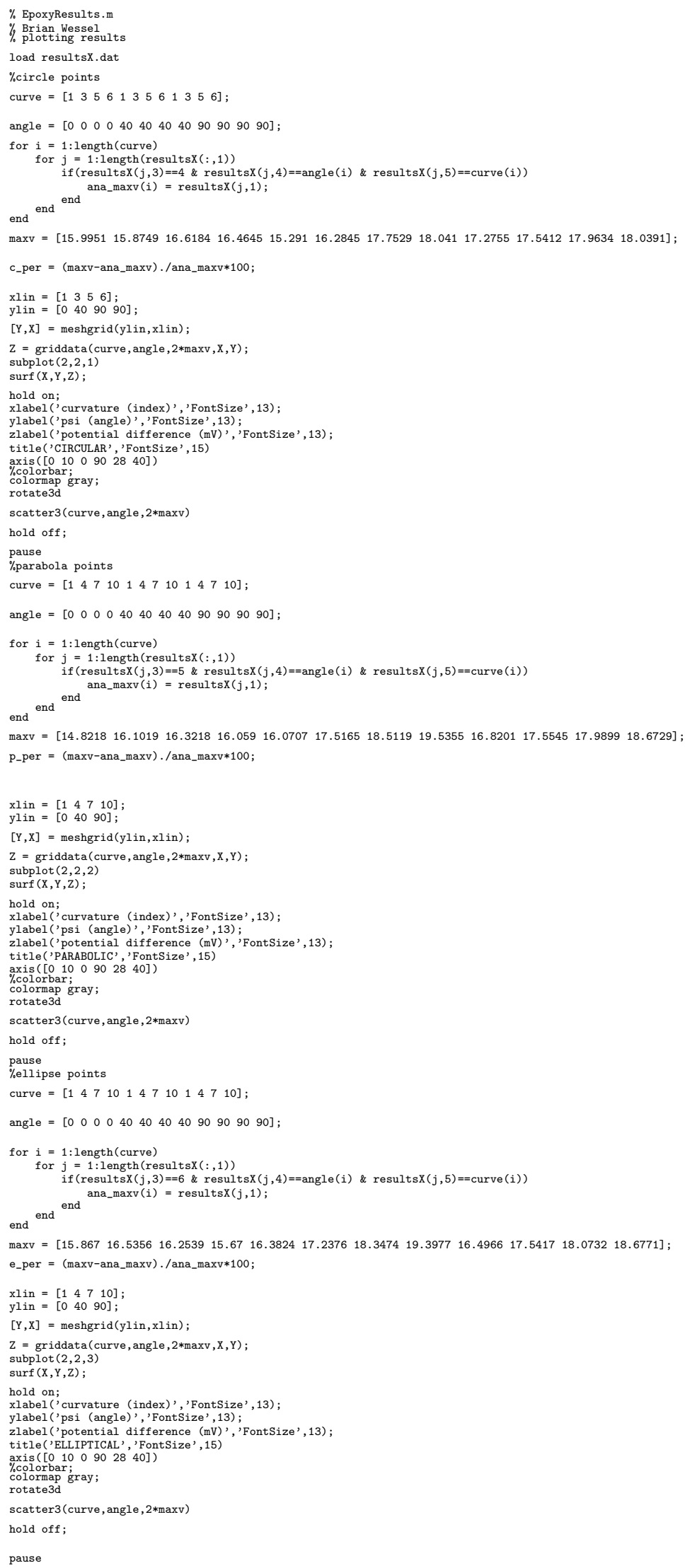




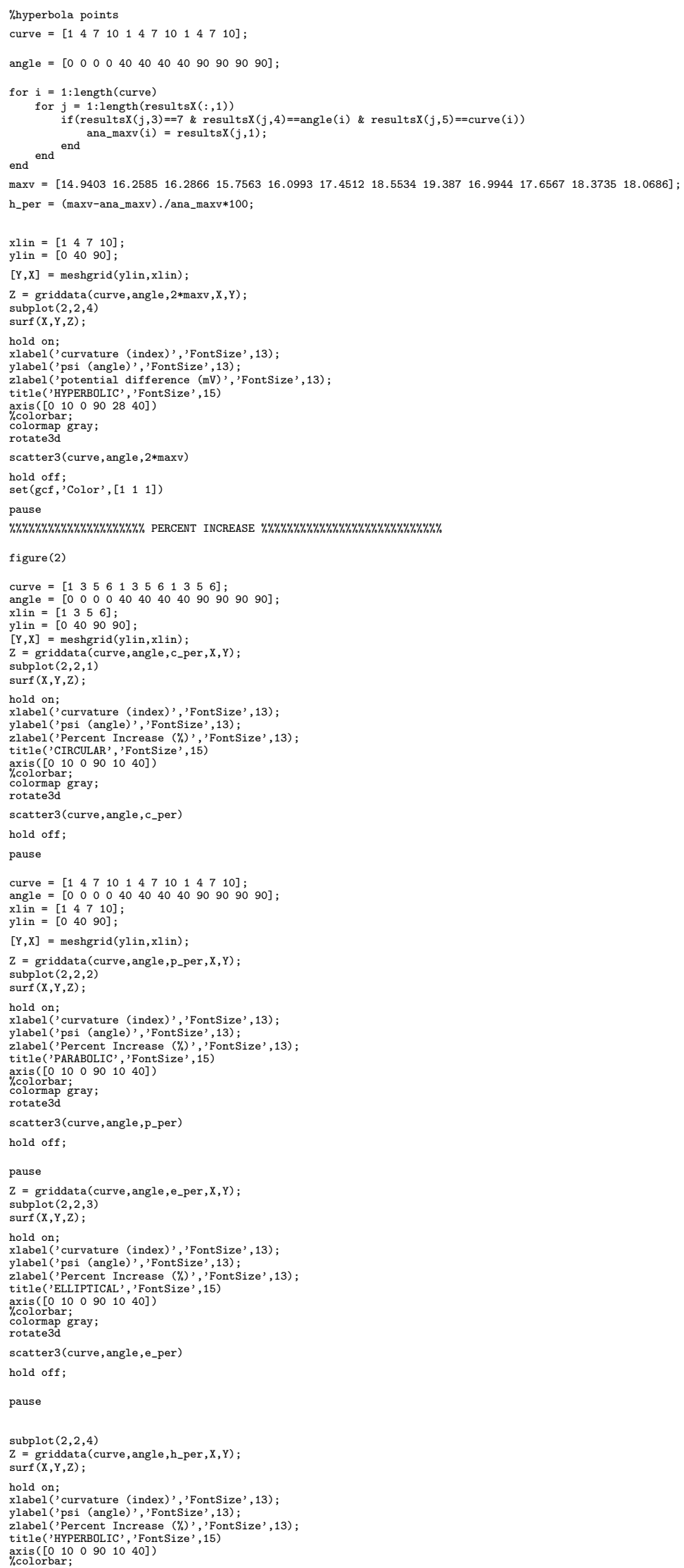


colormap gray;

scatter 3 (curve, angle, h_per)

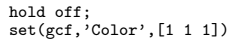




\section{.16 PLOTTING - NO EPOXY (FE) WITH \% INCREASE FROM ANALYTICAL}

Contains the following file:

- NoEpoxyResults.m 


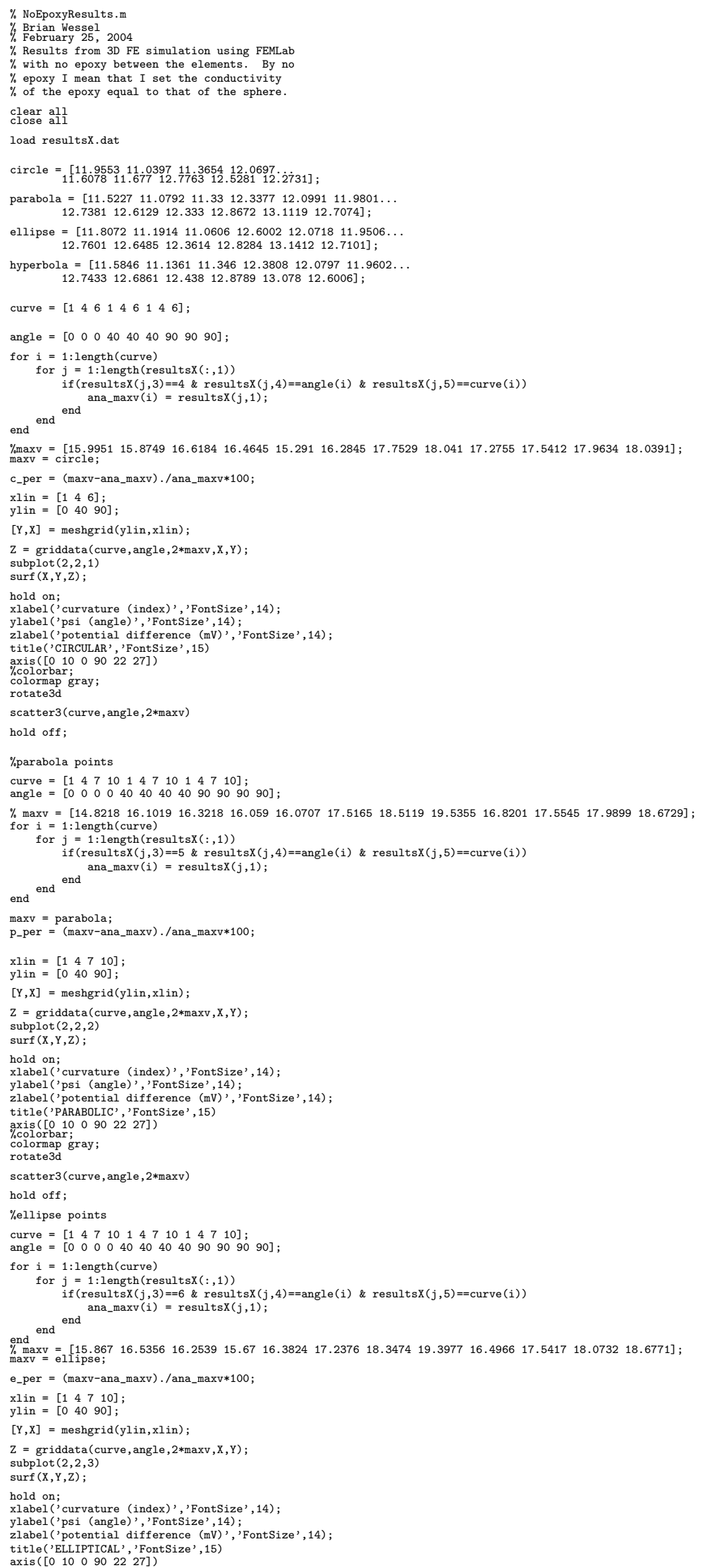




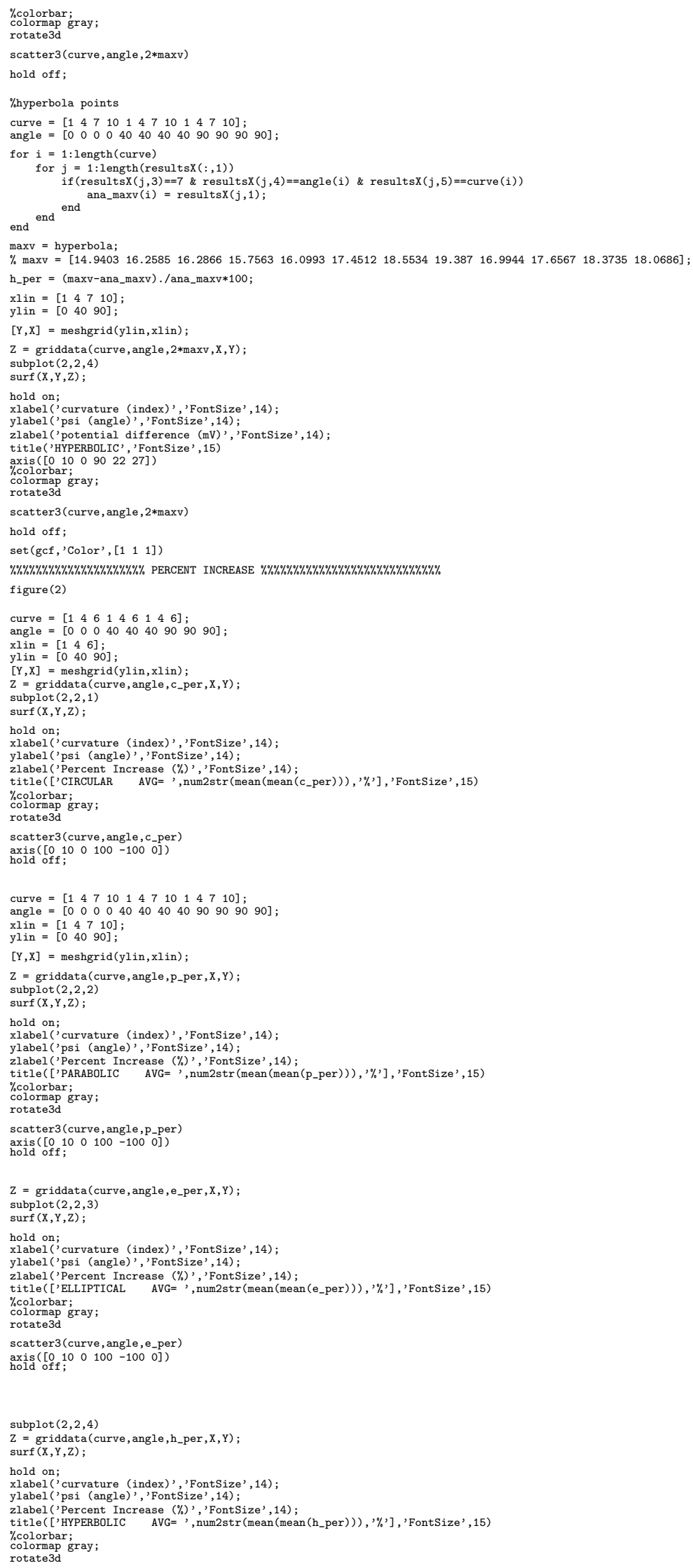


set (gca, 'FontSize', 12)

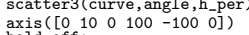

set (gcf, 'Color', [ [ [ $\left.\begin{array}{lll}1 & 1 & 1\end{array}\right]$ ) 


\section{.17 CONDUCTIVITY CONVERGENCE}

Contains the following file:

- CondConvergence.m 
$\%$ CondConvergence.m

$\%$ Brian Wessel 1 ptting results of experiment

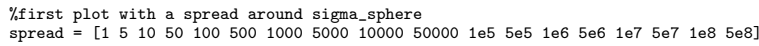

$\max V=\left[\begin{array}{llllll}13.6778 & 15.3221 & 15.7715 & 16.3031 & 16.3929 & 16.4507 \\ 16.4306 & 16.3742 & 16.3320 & 16.2570 & 16.2255 & 16.1354\end{array}\right]$

figure(1)

plot (log10 (spread), maxV, 'r.')

10 (spread)'

(spread)')

axis equal

[logio(spread)', maxV']

$\%$ second plot with a constant sigma_ae but decreasing sigma_ins

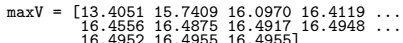

sig_sphere $=100 / 222 ;$
sig_ae $=$ sig_sphere $* 50$

sig_ae = sig_sphere $* 50 ;$
sig_ins $=$ sig_sphere./[1 5105010050010005000 1e4 5e4 1e5];

spread = sig_ae./sig_ins;

figure(2)

plot (log10 (spread), maxV, 'ro', 'MarkerSize', 10)

title('Max Voltage vs Log_\{10\} (\sigma_\{epoxy\}/Nigma_\{epoxy\})', 'FontSize', 20)

xlabel ('Log_\{10\} (\sigma_\{metal\}/Nsigma_\{epoxy\})', 'FontSize', 20)

ylabel( Max Voltage', 'FontSize',

set (gca, 'FontS
axis equal

$\%$ gtext ('\sigma_ $\{$ ae $\}=\backslash$ sigma_ $\{$ sphere $\} * 50$ ') 


\section{BIBLIOGRAPHY}

[1] Halliday D, Resnick R, and K Krane. Physics. Vol. 2, 4th ed., John Wiley \& Sons, Inc., NY, 1992.

[2] Hauser WA, Hesdorffer DC. Epilepsy: Frequency, Causes and Consequences. New York: Demos Press, 1990.

[3] Engel J Jr, Shewmon DA. Overview: who should be considered a surgical candidate? In: Engel J Jr, ed. Surgical Treatment of the Epilepsies 2nd ed. New York: Raven Press, 1993:23-24.

[4] Engel J Jr. A Greater Role for Surgical Treatment of Epilepsy: Why and When?. Epilepsy Currents. Vol. 3, No. 2 (March/April) 2003:37-40.

[5] Sun M, Mickle M, Liang W, Liu Q, Sclabassi RJ. Data Communication between Brain Implants and Computer. IEEE Transactions on Neural Systems and Rehabilitation Engineering. Vol. 11, No. 2, June 2003.

[6] Sun M, Liu Q, Liang W, Wessel BL, Roche P, Mickle M,Sclabassi RJ. Application of the Reciprocity Theorem to Volume Conduction Based Data Communication Systems between Implantable Devices and Computers. IEEE Transactions on Neural Systems and Rehabilitation Engineering, Vol. 11, No. 2, June 2003.

[7] Malmivuo J, Plonsey R. Bioelectromagnetism. Oxford University Press, New York, 1995.

[8] Rush S, Driscoll DA. EEG-electrode sensitivity-An application of reciprocity. IEEE Trans. Biomed. Eng.. BME-16:(1) 15-22.

[9] Barber DC, Brown BH. Applied potential tomography. J. Phys. E.: Sci. Instrum. Vol. 17: 723-733.

[10] Plonsey R, Heppner DB. Considerations of quasi-stationarity in electrophsyiological systems. Bulletin of Mathematical Biophysics Vol. 29, 1967, 657-664.

[11] Webster, JG ed. Medical Instrumentation: Application and Design. 3rd ed.,John Wiley \& Sons, Inc. New York, 1998. 
[12] R. D. Sidman, V. Giambalvo, T. Allison and P. Bergey . "A method of localization of sources of human cerebral potentials evoked by sensory stimuli" Sensory Processes Vol. 2, 1978, pp. 116-129.

[13] Frank, E. "Electric potential produced by two point current sources in a homogeneous conducting sphere," J. Appl. Phys. 23: 1225-1228 (1952).

[14] FEMLab Reference Manual.COMSOL, Nov, 2001, pp 3-124,5-287.

[15] Sun M." An Efficient algorithm for computing multishell spherical volume conductor models in EEG dipole source localization," IEEE Transactions on Biomedical Engineering. Vol. 44, No. 12, December, 1997.

[16] Chari MVK, Salon SJ. Numerical Methods in Electromagnetism. Academic Press, San Diego, 2001.

[17] Gulrajani RM. Bioelectricity and Biomagnetism. John Wiley \& Sons, Inc. New York, 1998.

[18] http://www.mathworks.com/access/helpdesk/help/toolbox/pde/4fem2.shtml.

[19] Hunter, P and A Pullan. FEM/BEM Notes. The University of Auckland, New Zealand. (C)1997-2003.

[20] Ahonen, AI, Hamalainen MS, Ilmoniemi RJ, Jajola MJ, Knuutila JET, Simola JT, Vilkman VA. ."Sampling Theory for Neuromagnetic Detector Arrays." IEEE Transactions on Biomedical Engineering. Vol. 40, No. 9, September, 1993.

[21] Lindsey DP, McKee EL, Hull ML, Howell SM. ."A New Technique for Transmission of Signals from Implantable Transducers." IEEE Transactions on Biomedical Engineering . Vol. 45, No. 5, May, 1998.

[22] Cuffin BN and D Cohen. "Comparison of the magnetoencephalogram and electroencephalogram." Electroencephalography and Clinical Neurophysiology . vol. 47, pp 132-146, 1979.

[23] Lindsey DP, McKee EL, Hull ML, Howell SM. ."A New Technique for Transmission of Signals from Implantable Transducers." IEEE Transactions on Biomedical Engineering . Vol. 45, No. 5, May, 1998. 